\title{
Molecular Dynamics Study of Zirconium and Zirconium Hydride
}

\author{
A Thesis Submitted to the College of \\ Graduate Studies and Research \\ In Partial Fulfillment of the Requirements
}

For the Degree of Master of Science

In the Department of Mechanical Engineering

University of Saskatchewan

Saskatoon

By

Ravi Kiran Siripurapu

(C) Copyright Ravi Kiran Siripurapu, October, 2013. All rights reserved. 


\section{Permission to Use}

In presenting this thesis/dissertation in partial fulfillment of the requirements for a Postgraduate degree from the University of Saskatchewan, I agree that the Libraries of this University may make it freely available for inspection. I further agree that permission for copying of this thesis/dissertation in any manner, in whole or in part, for scholarly purposes may be granted by the professor or professors who supervised my thesis/dissertation work or, in their absence, by the Head of the Department or the Dean of the College in which my thesis work was done. It is understood that any copying or publication or use of this thesis/dissertation or parts thereof for financial gain shall not be allowed without my written permission. It is also understood that due recognition shall be given to me and to the University of Saskatchewan in any scholarly use which may be made of any material in my thesis/dissertation.

\section{Disclaimer}

Reference in this thesis/dissertation to any specific commercial products, process, or service by trade name, trademark, manufacturer, or otherwise, does not constitute or imply its endorsement, recommendation, or favoring by the University of Saskatchewan. The views and opinions of the author expressed herein do not state or reflect those of the University of Saskatchewan, and shall not be used for advertising or product endorsement purposes.

Requests for permission to copy or to make other uses of materials in this thesis/dissertation in whole or part should be addressed to:

Head of the Department of Mechanical Engineering

University of Saskatchewan

57 Campus Drive

Saskatoon, Saskatchewan S7N 5A9

Canada

OR

Dean

College of Graduate Studies and Research

University of Saskatchewan

107 Administration Place

Saskatoon, Saskatchewan S7N 5A2

Canada 


\section{ABSTRACT}

Molecular dynamics (MD) simulations were used in order to investigate structure and mechanical properties of zirconium and zirconium hydride. Calculation of temperature dependent failure of zirconium, diffusion of hydrogen in zirconium, properties of interfaces in zirconium and zirconium hydride and effect of hydrogen on crack nucleation and propagation were in good agreement with available experimental data. These are the first computer simulations where large-scale atomic/molecular massively parallel simulator (LAMMPS) code was used with the Embedded Atom Method (EAM) and Modified Embedded Atom Method (MEAM) to study structure and mechanical properties of zirconium hydrogen system $(\mathrm{Zr}-\mathrm{H})$ and zirconium hydride $\left(\mathrm{ZrH}_{2}\right)$.

Verification of methods was done in order to establish the best potential for zirconium and zirconium hydride. EAM and MEAM potentials successfully predicted lattice parameters, mechanical properties and variation of lattice parameters with temperature for $\alpha$-Zr. MEAM potential was used to predict correctly the face centered structure for $\mathrm{ZrH}_{2}$ and also its mechanical properties.

Temperature dependent stress-strain curves were calculated in order to predict yielding point for $\alpha-Z r$. Results indicate early yielding and failure with increase of temperature in zirconium on application of tensile and compressive strains. Anisotropic stress variation with temperature in $\alpha$ Zr was calculated.

Hydrogen ingress through diffusion of hydrogen in zirconium is a mechanism responsible for formation of hydrides. Temperature-dependent hydrogen diffusion and activation energy for diffusion was calculated and the agreement with experiments was satisfactory. Anisotropy of diffusion of hydrogen is observed for $\mathrm{Zr}$ crystal. Hydrogen diffusion was also modeled under tensile and compressive strain and a possible formation of hydrides in the direction perpendicular to applied strain was observed.

The effect of strain on orientation of hydride was investigated. Hydride $\{111\}$ oriented crystal was strained along [ $\left[\begin{array}{lll}1 & \overline{1} & 0\end{array}\right]$ and [111] direction. Energy as a function of strain is calculated along 
both directions [111] and [ $\left.\begin{array}{lll}1 & \overline{1} & 0\end{array}\right]$ and it was found that energy of the system increase with increase in strain along [1 $\left.\begin{array}{lll}1 & 0\end{array}\right]$ and decrease with increase of strain along [111] direction. Calculated stress and strain curves indicate lower stresses along [111] direction and this causing the hydride to reorient in a direction perpendicular to applied strain.

Structure of the interface $\left(\begin{array}{llll}0 & 0 & 0 & 1\end{array}\right) \alpha-\mathrm{Zr} / /\left[\begin{array}{lll}1 & 1 & 1\end{array}\right\} \delta-\mathrm{ZrH}_{2}$ is modeled in order to investigate the crack initiation at this interface. Interfacial cracking of hydride under stress is observed. This observation is in good agreement with available experimental studies. Cracks are seen to nucleate earlier at higher temperature.

Cracks and voids are common defects in zirconium fuel cladding. A crack is modeled along ( 00 01 ) plane of zirconium with hydrogen. In the presence of hydrogen cracks nucleate in zirconium causing fracture. This observation is in good agreement with previous experimental studies.

Bonds surrounding atoms and stress concentration analysis were performed using OVITO and VMD software respectively. Weaker bonds and higher stress concentrations are observed in the presence of hydrogen in zirconium. The presented results clearly demonstrate that MD simulation can be used to predict structure and processes that are important for understanding failure in $\mathrm{Zr}$ based nuclear materials. 


\section{PREFACE}

Sections of this thesis have been submitted as a multi-authored paper in refereed conference papers. The research, data analyses and manuscript preparation were carried out by me and the co-authors contributed in editing the manuscripts for submission to refereed conference papers.

Author's contributions: - Ravi Kiran Siripurapu, was jointly responsible for the original ideas developing the model, image and mechanical data analyses, presentation of the findings, and writing and editing of the original paper. Dr. Barbara Szpunar was jointly provided supervision, required literature, and edited the paper. Dr. Jerzy A. Szpunar was jointly involved interpretation of study findings and editing of the paper.

The results in this study were presented at local and national conferences

1. Siripurapu R., Szpunar J.A. and Szpunar B., Proc. of the 8th Pacific Rim International Congress on Advanced Materials and Processing, L. Modeling and Simulation of Processes, Microstructures, and Behavior, 3119-3126, Ed. F. Marquis, TMS, Wiley, August 4-9, 2013, Waikoloa, Hi, Molecular Dynamics Study of Zirconium and Zirconium Hydride.

2. Siripurapu, R., Szpunar B., and Szpunar J.A., "Investigation of Interfacial Cracking of Hydride in Zirconium", 24th Canadian Congress of Applied Mechanics, Saskatoon, Canada, 68, June 3-5 (2013).

3. Siripurapu, R., Szpunar B., and Szpunar J.A., "Molecular Dynamics Simulation of Zirconium and Zirconium Hydride", 36th Annual CNS/CNA Student poster Conference, Saskatoon, Canada, 122, June 10-13 (2012). 
Acknowledgement

I would like to express the deepest appreciation to my supervisors, Prof Dr. Jerzy A. Szpunar and Dr. Barbara Szpunar, for all their support. My special thanks go to Dr. Barbara Szpunar for her guidance and support this thesis would not have been possible. She was patient while I was learning how to conduct research and supported me at every step of my work. I thank Prof Dr. Jerzy A. Szpunar for his supervision, good advice, support and friendship that has been invaluable on both academic and personal level.

I would like to thank Compute Canada/ Calcul Canada for providing me access to high performance computing (HPC). I also thank NSERC for proving financial assistance for this research. I would like to thank Westgrid support team for providing me computational facilities and answering my questions on both Bugaboo and Zeno computers where my research is conducted.

My sincerest thanks go to my dearest Mom, Dad, brother and my sister for their heartwarming love and encouragement throughout all of my university studies.

Many thanks to Dr. Nilesh Gurao and Dr. N.A.P Kiran Kumar for their insightful discussions, comments about my work and for being there for me when I needed help. My appreciation goes to my friends at the Laboratory, for their support and for making my time here more enjoyable. 


\section{Table of Contents}

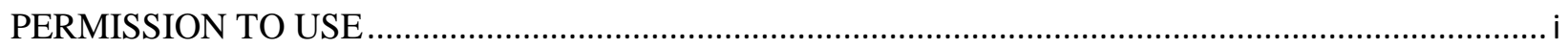

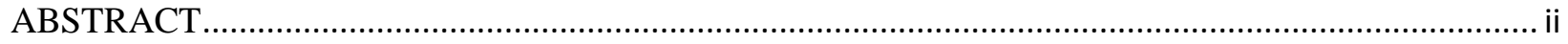

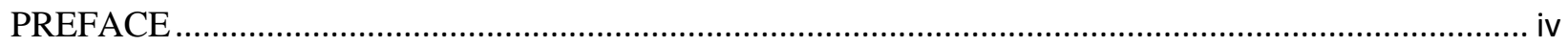

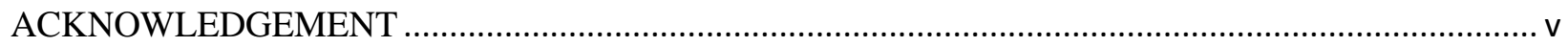

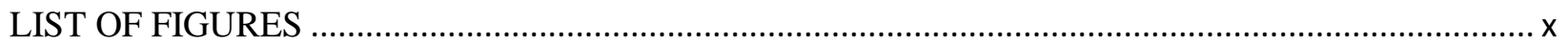

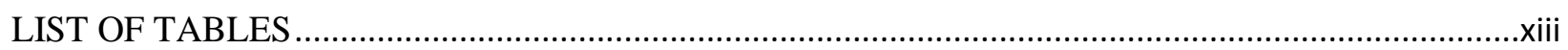

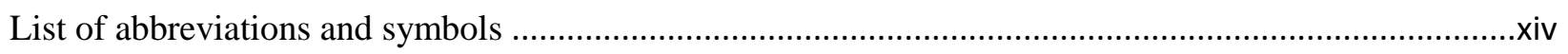

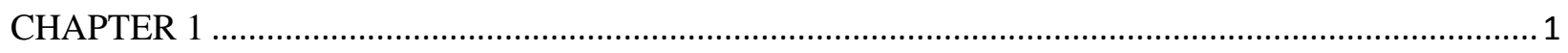

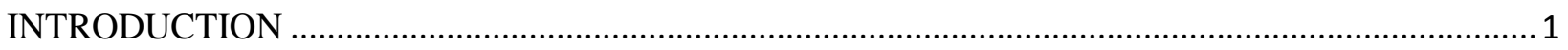

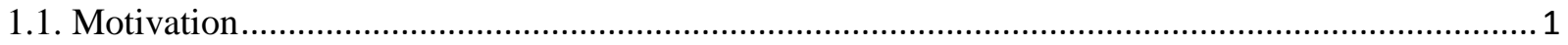

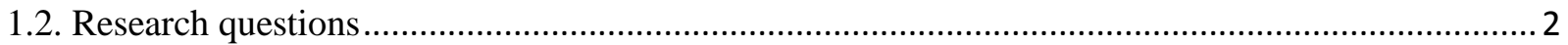

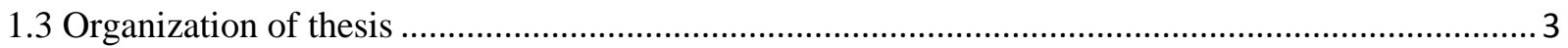

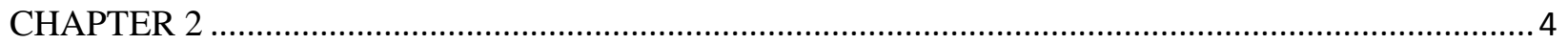

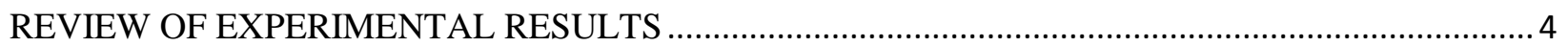

2.1. Experimental lattice parameters of zirconium ....................................................................... 4

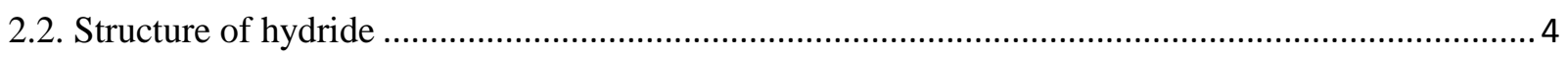

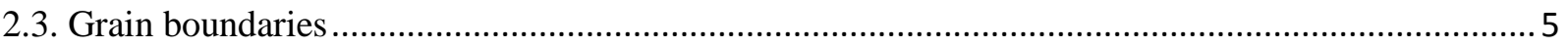

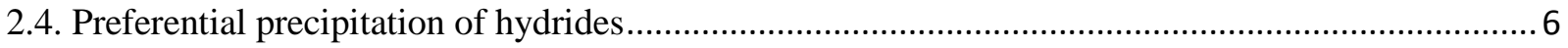

2.5. Reorientation of zirconium hydrides under stress ..................................................................... 6

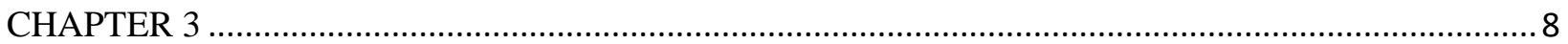

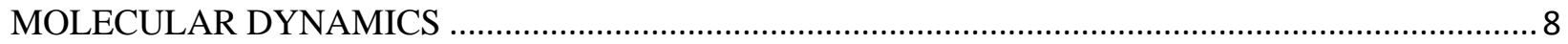

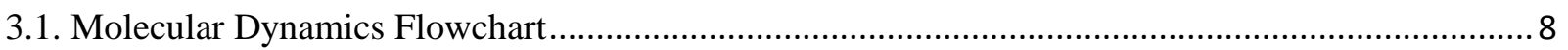

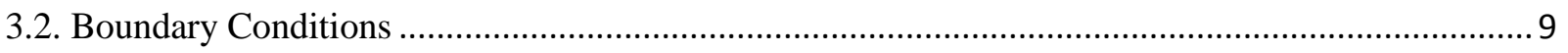

3.3. Significance of potentials in Molecular Dynamics ................................................................. 11

3.4. Embedded Atom Method (EAM) ……….............................................................................. 11

3.5. Modified Embedded Atom Method (MEAM) ........................................................................ 12

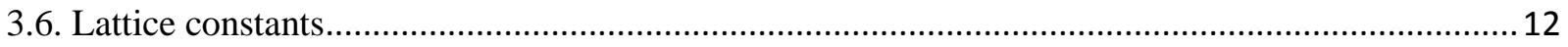

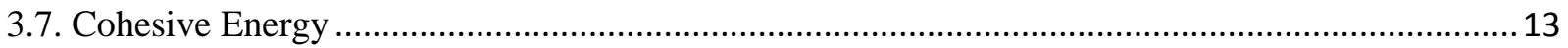

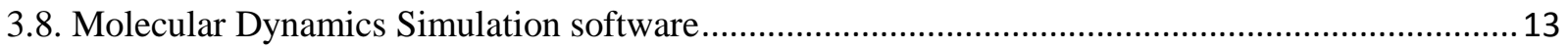

3.8.1. LAMMPS: - large-scale atomic/molecular massively parallel simulator ............................... 13

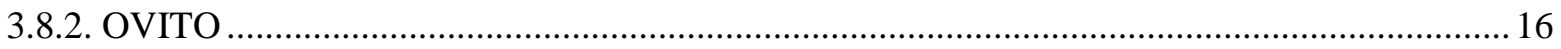




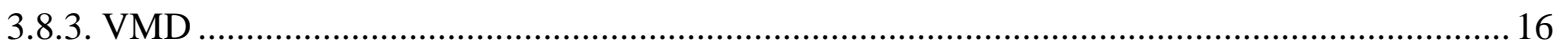

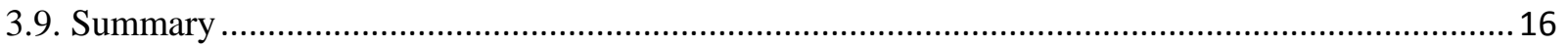

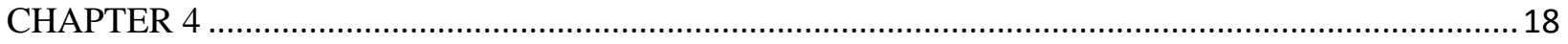

VERIFICATION COMPUTER MODELS AND SIMULATION METHODS ................................... 18

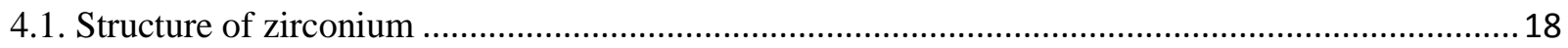

4.1.1 Lattice parameters for zirconium using EAM and MEAM potentials ................................... 18

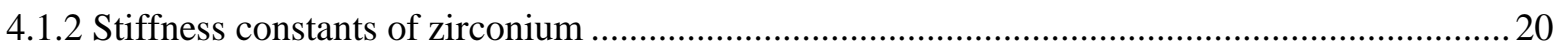

4.1.3. Comparison of Young's Modulus and Poisson's ratio for EAM, MEAM...............................2 21

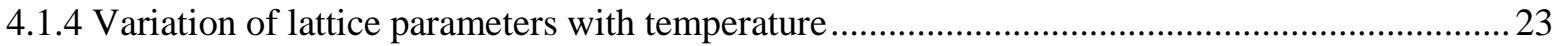

4.1.5. Anisotropic thermal expansion in zirconium using EAM and MEAM ................................. 24

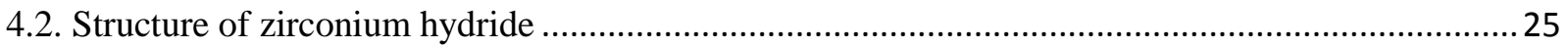

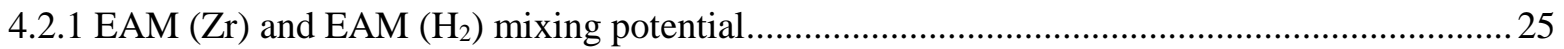

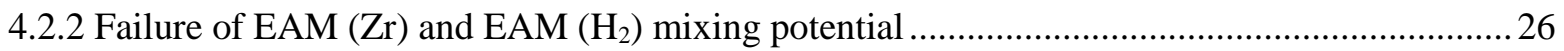

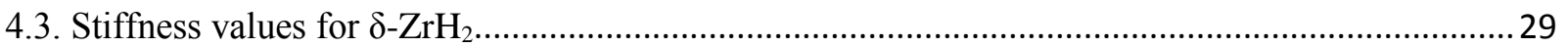

4.4. Variation of Lattice parameter with Temperature ..................................................................... 31

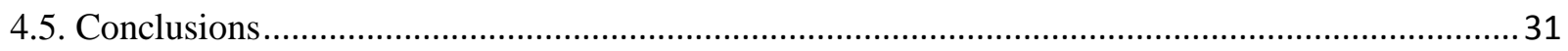

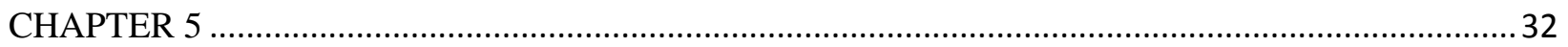

STRESS ANALYSIS IN ZIRCONIUM USING MEAM AND EAM POTENTIALS..............................32

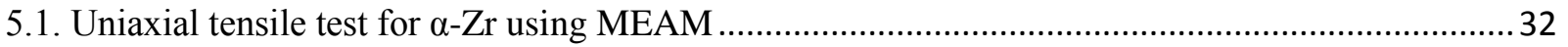

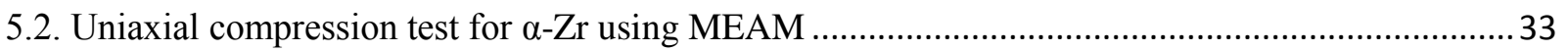

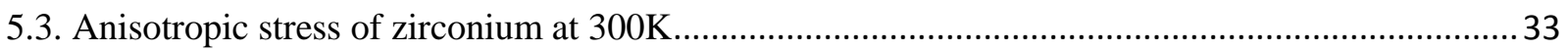

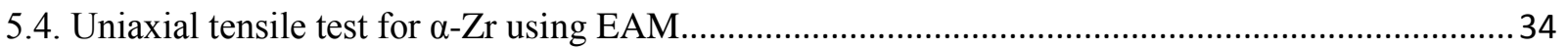

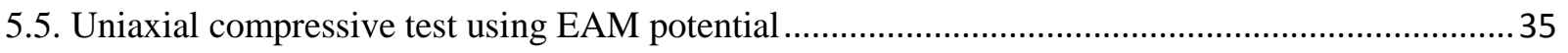

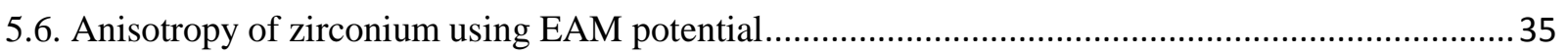

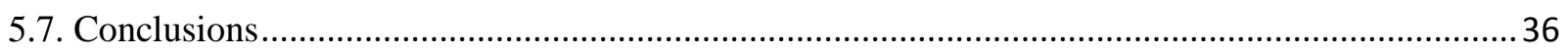

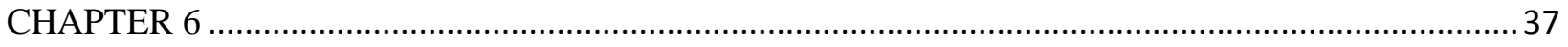

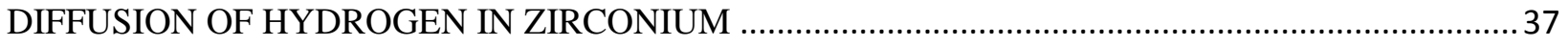

6.0. Diffusion of hydrogen in zirconium using EAM and MEAM ..................................................... 37

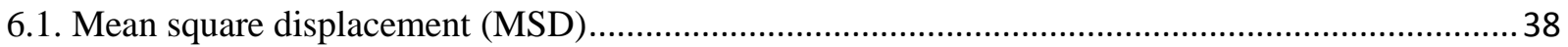

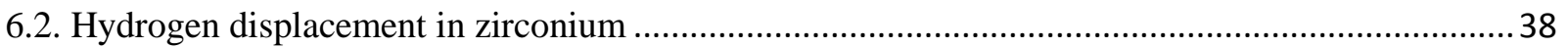

6.3. Diffusion coefficients of hydrogen and activation energy of hydrogen....................................... 40

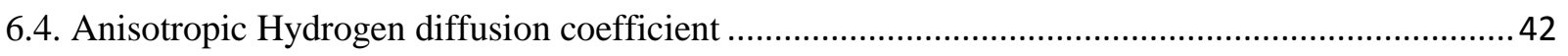

6.5. Strain induced diffusion of hydrogen in zirconium ............................................................. 42 


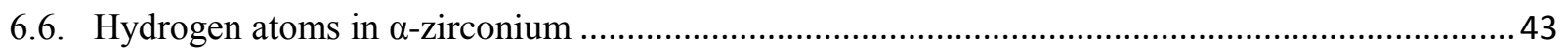

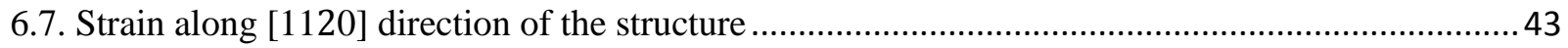

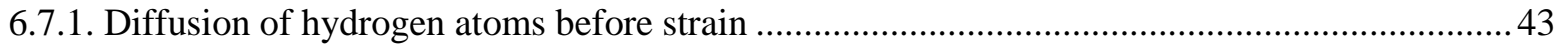

6.7.2 Calculation of Diffusion under uniaxial strain along [1120] direction .................................... 44

6.7.3 Diffusion calculation under compression along [1120] direction..........................................45

6.8. Strain along [0001] direction of the structure ....................................................................... 46

6.8.1. Calculation of diffusion under uniaxial tensile strain along [0001] direction.......................... 46

6.8.2 Hydrogen diffusion calculation under compression stress along [0001] direction ...................47

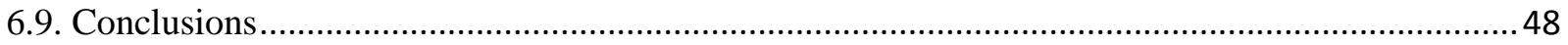

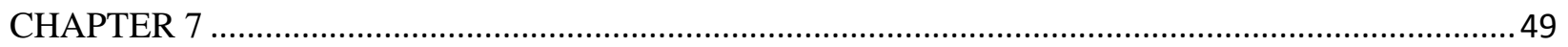

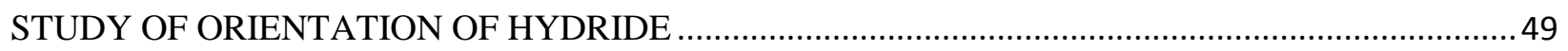

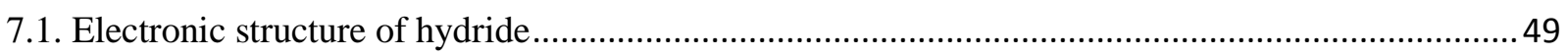

7.2. Uniaxial tensile strain applied to hydride along different crystal directions.................................50

7.3. Uniaxial tensile stress and strain analysis on hydride ............................................................... 50

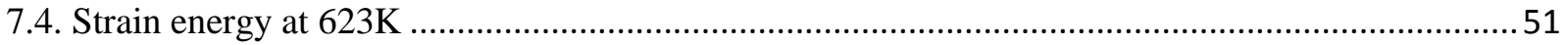

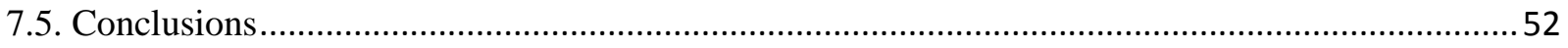

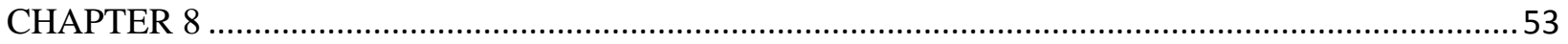

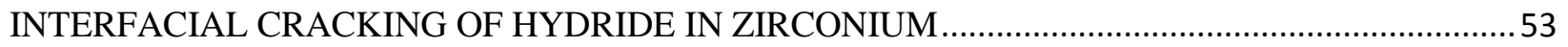

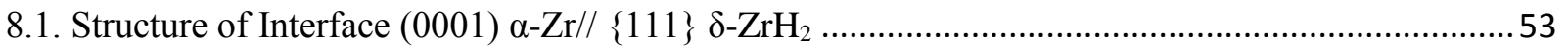

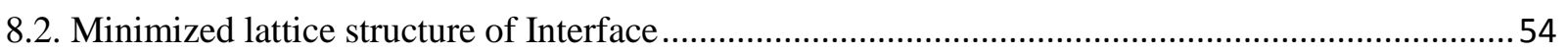

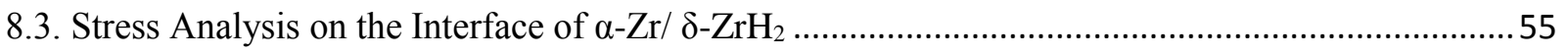

8.4. Stress analysis on Interface at various operating conditions ...................................................... 56

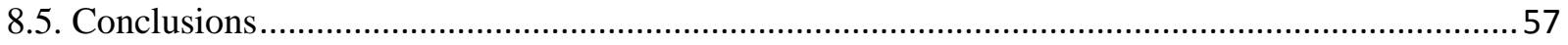

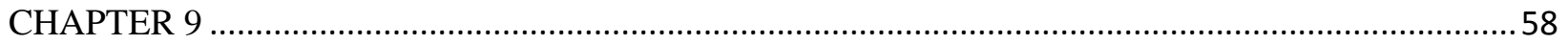

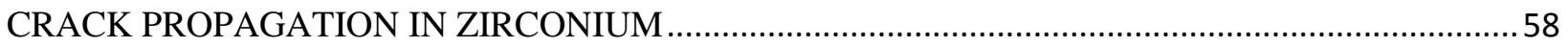

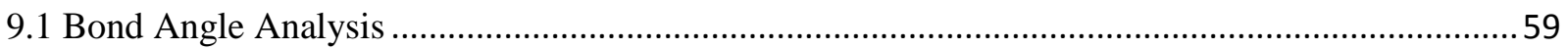

9.2. Crack propagation in zirconium at different temperatures and timesteps ....................................59

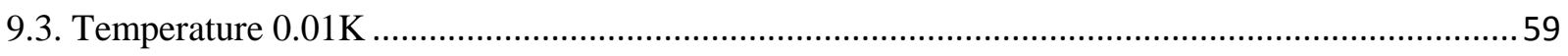

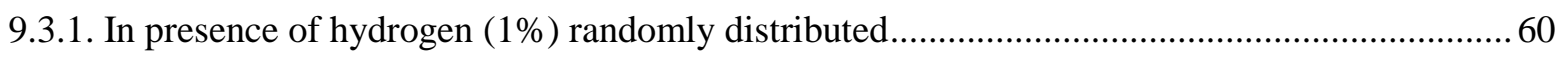

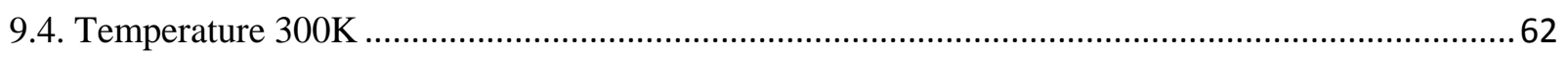

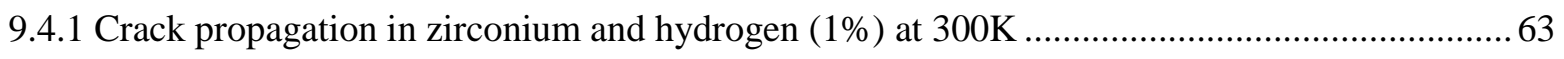

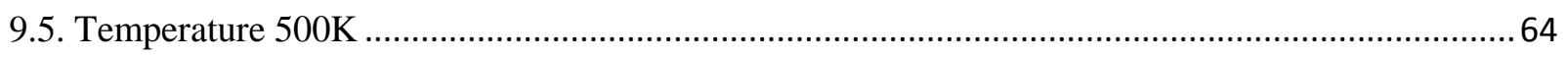

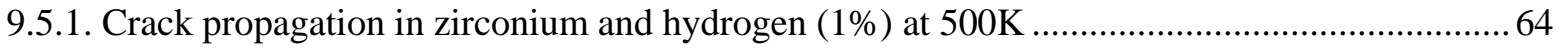




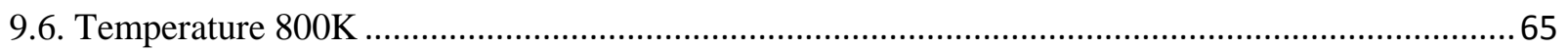

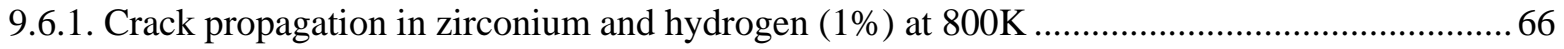

9.7. Study of stress concentration in $\alpha-Z r$ in presence of hydrogen using VMD software ....................66

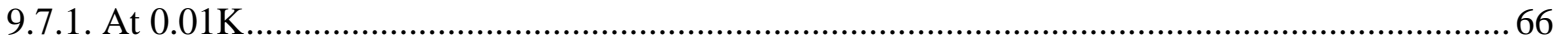

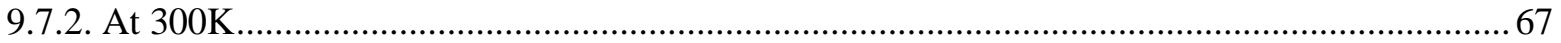

9.7.3. At 500K

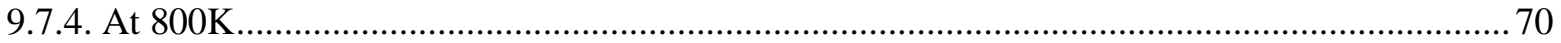

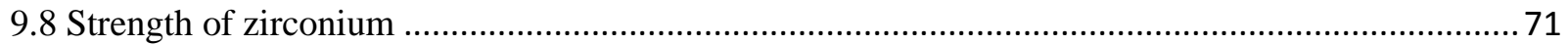

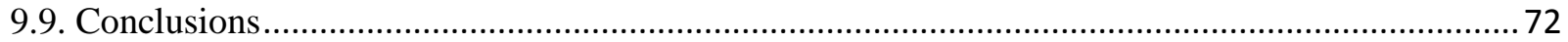

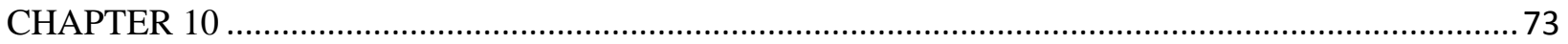

SUMMARY OF RESULTS, LIMITATIONS AND FUTURE WORK …............................................... 73

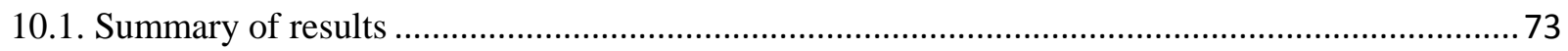

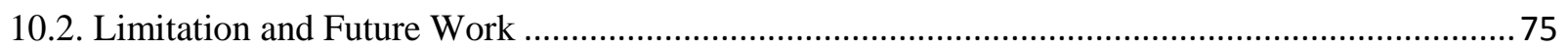

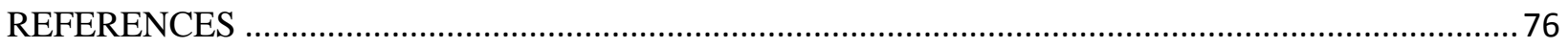

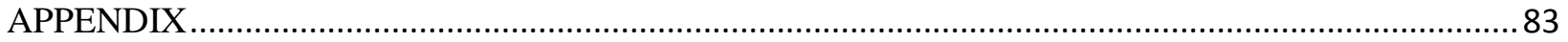




\section{List of Figures}

Figure 1: Cracks caused due to hydrogen embrittlement in zirconium

Figure 2: Variables that define a grain boundary $\mathrm{xA} ; \mathrm{yA} ; \mathrm{zA}$ and $\mathrm{xB}, \mathrm{yB}, \mathrm{zB}$ are the axes of the co-ordinates parallel to crystallographic directions in grains $\mathrm{A}$ and $\mathrm{B}$, respectively. $\mathrm{o}$ is the rotation axis and $\theta$ is the rotation (misorientation) the angle necessary to rotate one grain to orientation of another $\mathrm{n}$ determines the orientation of the grain boundary plane...... 5

Figure 3: EBSD image of preferential precipitation of hydrides at grain boundary, orientation relation illustrated by pole figures

Figure 4 : Schematic view of a) hydride orientation without hoop stress and b) hydride orientation under stress

Figure 5: Periodic boundary conditions $(\mathrm{PBC})$ is where atoms leave from one box and enter into another box keeping the total number of atoms constant and the area shaded yellow is the area of interest.

Figure 6: Boundary conditions that can be used in molecular dynamics. (a) Free surface boundary condition where atoms leave the surface. (b) Rigid surface where atoms shown in yellow act as a wall and do not move with time used for simulating fluid mechanics. (c) Flexible boundary condition are used to make boundaries rigid enough to maintain structure and flexible enough to interact with the other atoms. 10

Figure 7: (a) Unit cell HCP. (b) Atomic arrangement of $\mathrm{Zr}$.................................................. 18

Figure 8: Convergence of EAM potential (a) Volume convergence (b) Energy convergence.... 19 Figure 9: Convergence of MEAM potential (a) Volume convergence (b) Energy convergence 19 Figure 10: (a) and (b) Stress and strain directions on cubic crystal in different directions ......... 21

Figure 11: Directions of hexagonal crystal in which anisotropy is calculated .......................... 22 Figure 12: (a) Variation of lattice parameter 'a' with temperature (b) Variation of lattice parameter 'c' with temperature. 25 Figure 13: Structure of face centered hydride zirconium (red) and hydrogen (blue) in tetrahedral

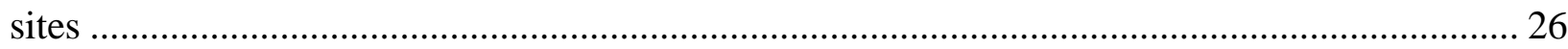

Figure 14: Minimized energy for hydride using EAM potential .............................................. 26 Figure 15: Minimized lattice parameters for hydride using EAM potential indicates unstable

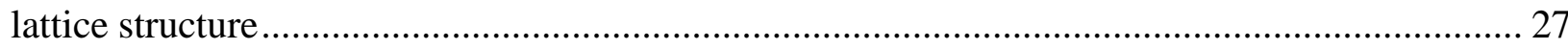

Figure 16: Convergence of potential energy for face centered hydride (fct) using MEAM potential.

Figure 17: Convergence of lattice parameters for fct to fcc hydride using MEAM potential along 'a' and 'c' directions of the tetragonal face centered cubic crystal ....................................... 28 Figure 18: Convergence of the potential energy for face centered cubic (fcc) using MEAM...... 28

Figure 19: Face centered cubic structure hydride showing different crystal directions .............. 29 Figure 20: Variation uniaxial tensile stress for zirconium using MEAM potential at different

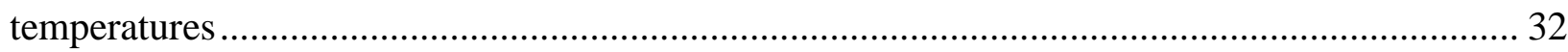
Figure 21: Variation uniaxial compressive stress for zirconium using MEAM potential at

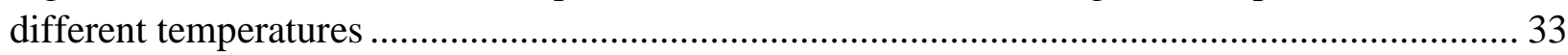

Figure 22: Anisotropic variation of stress for zirconium at constant temperature. .................... 34 
Figure 23: Uniaxial tensile stress-strain curves of zirconium calculated for different temperatures using EAM potential ................................................................................. 34 Figure 24: Uniaxial compression test on zirconium using EAM potential indicate early yielding

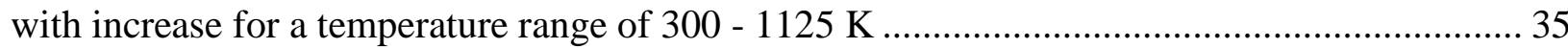
Figure 25: Stress-strain curves in different directions of zirconium calculated using EAM

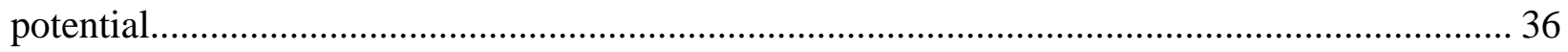
Figure 26 : Hydrogen (blue) is distributed randomly in zirconium (brown) ............................ 38 Figure 27: Variation of mean square displacement (MSD) of hydrogen inside zirconium for a temperature range of (500 -1200) K. Graph indicates hydrogen jumps (variation in slope) inside zirconium 39

Figure 28: At $1200 \mathrm{~K}$ unstable structure using MEAM (b) EAM shows perfectly stable structure and higher displacements of hydrogen. 40 Figure 29: Arrhenius plot of hydrogen in zirconium for a temperature range of $(500-1200) \mathrm{K}$ indicates good diffusion coefficients of hydrogen when compared with the experimental data.. 41 Figure 30: Anisotropic hydrogen diffusion coefficient's inside zirconium with increase in temperature

Figure 31: Different orthogonal views of hydrogen atoms (blue) placed in spherical box inside zirconium (brown) in order to study hydrogen diffusion with strain.

Figure 32: Hydrogen displacement is similar in all directions during equilibration and when no strain is applied

Figure 33: Mean square displacement of hydrogen atoms in 3 different directions of zirconium when applying the tensile strain along [1120].Jump in MSD indicate movement of hydrogen atoms.

Figure 34: Mean square displacement of hydrogen atoms in 3 different directions of zirconium on compression along [1120].Higher jumps in MSD is seen along [0 $\left.\begin{array}{llll}0 & 0 & 0 & 1\end{array}\right]$ and $\left[\begin{array}{llll}1 & 1 & 0 & 0\end{array}\right]$ when structure is compressed.

Figure 35: Mean square displacement of hydrogen atoms for tensile strain applied along [0001] direction in zirconium. Jumps are seen to be higher along [1120] and [1100] due to movement of hydrogen in that direction.

Figure 36: Mean square displacement of hydrogen atoms in 3 directions when compressed along [0001] direction of zirconium. Higher jumps in MSD indicate movement of hydrogen atoms along [1120] and [1 1100$]$

Figure 37: Hydride orientation along $\{111\}$ plane, zirconium is marked as brown, hydrogen is marked as blue.

Figure 38: Energy variation with strain along [ $\left[\begin{array}{lll}1 & 1 & 0\end{array}\right]$ and [111] crystal directions of hydride ... 50 Figure 39: Stress-strain variation along [ $\left[\begin{array}{lll}1 & 1 & 0\end{array}\right]$ and [111] crystal directions of hydride............. 51 Figure 40: Energy difference in hydride along [ $\left[\begin{array}{lll}1 & 1 & 0\end{array}\right]$ and [111] is seen to increase with strain at $623 \mathrm{~K}$

Figure 41: Atomic arrangement of interface (0001) $\alpha-\mathrm{Zr} / /\{111\} \delta-\mathrm{ZrH}_{2}$ with zirconium (brown) and hydrogen (blue) as per experimental data ................................................................ 54 Figure 42: Relaxed Interface (0001) $\alpha-\mathrm{Zr} / /\{111\} \delta-\mathrm{ZrH}_{2}$ with zirconium (brown) and hydrogen

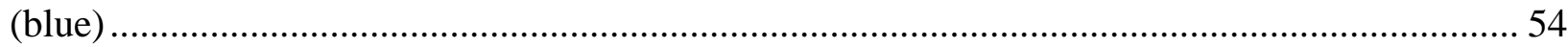
Figure 43: Energy convergence of Interface using MEAM potential...................................... 55 
Figure 44: Crack initiation hydride................................................. 57

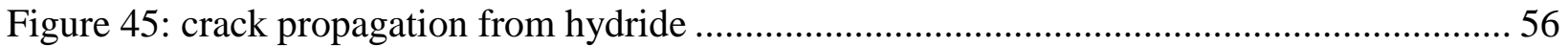

Figure 46: N.A.P. Kiran Kumar, J. A. Szpunar, SEM image small cracks developing in $\delta-\mathrm{ZrH}_{2}$

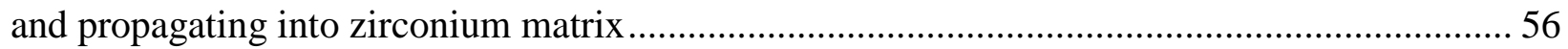

Figure 47: Increase in temperature nucleates crack early in Interface.................................. 57

Figure 48: Crystal structure of zirconium crack along (0001) plane ...................................... 58

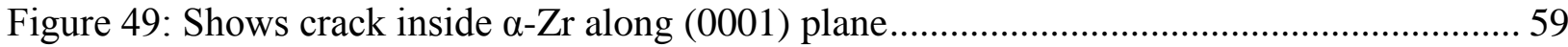

Figure 50: Crack propagation inside $\alpha-\mathrm{Zr}$ after 100 time steps timestep.................................... 60

Figure 51 Crack inside $\alpha-Z r$ and with randomly distributed hydrogen atoms (blue) ................. 61

Figure 52: Weaker bonds surrounding hydrogen atoms (white color) inside $\alpha-\mathrm{Zr}$.................. 61

Figure 53: Crack propagation in presence of hydrogen inside $\alpha-\mathrm{Zr}$ at 25 time steps ................ 61

Figure 54: Crack propagation in the presence of hydrogen in $\alpha-\mathrm{Zr}$ at 50 time steps fracturing of

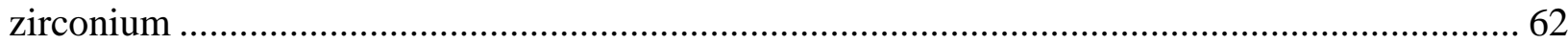

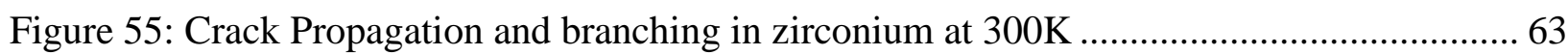

Figure 56: Crack propagation and branching in zirconium in presence of hydrogen at 300K ..... 63

Figure 57: Crack propagation and branching in zirconium at 500K .................................... 64

Figure 58: Crack propagation and branching in zirconium in presence of hydrogen at 500K ..... 65

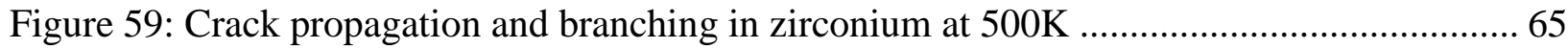

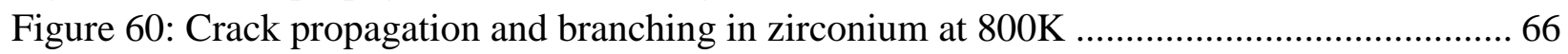

Figure 61: High stresses are seen ahead of crack causing crack to propagate in pure $\alpha-\mathrm{Zr}$....... 67

Figure 62: Crack propagation and failure of $\alpha \mathrm{Zr}$ is observed due to presence of hydrogen ....... 67

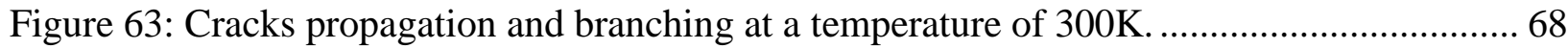

Figure 64: High crack blunting of crack in presence of hydrogen at 300K.............................. 68

Figure 65: High stresses surrounding crack and also ahead of crack at 500K ...................... 69

Figure 66: Increased crack blunting and crack elongation .................................................. 69

Figure 67: Crack branching on deformed zirconium due to high temperatures ....................... 70

Figure 68: Enhanced crack blunting with high deformation in zirconium due to hydrogen ........ 70

Figure 69: Indicates early crack nucleation in presence of hydrogen and loss of strength in

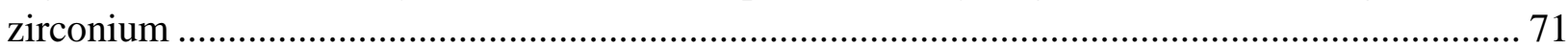

Figure 70: Stress reduction and crack nucleation with increase in temperature in presence of hydrogen 


\section{List of Tables}

Table 1: Variation of lattice parameter with temperature with zirconium................................ 4 Table 2: Lattice parameters and cohesive energy for zirconium calculated using EAM and MEAM potentials in comparison with experimental values................................................... 20 Table 3 Stiffness values for $\alpha-Z r$ using EAM, MEAM, and comparison with Experimental values

Table 4: Young's Modulus and Poisson ratio in comparison with experimental values.............. 23 Table 5: Lattice parameters changes with temperature calculated for EAM and MEAM potentials

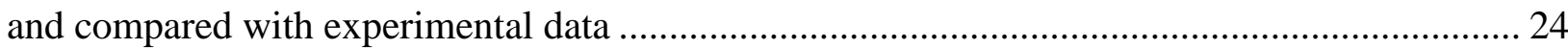
Table 6: Comparison of lattice parameters $(\AA)$ and cohesive energy (eV/atom) with experimental

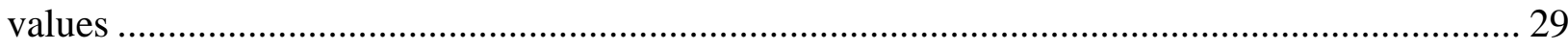

Table 7.Stiffness constants for $\delta-\mathrm{ZrH}_{2}$ structure and comparison with theoretical data.............. 30 Table 8: Young's Modulus and Poisson's ratio of zirconium hydride ..................................... 30 Table 9: Calculated mechanical properties in comparison with theoretical calculations ............ 30 Table 10 : Calculated activation energy of hydrogen in zirconium is within the range of observed

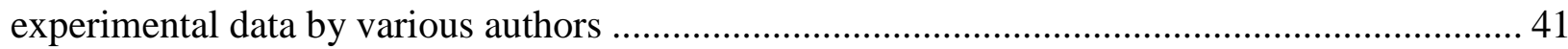

Table 11: Comparison of diffusion coefficient's on tension and compression strains. ............... 46

Table 12 Diffusion coefficient of tension and compression ................................................. 47

Table 13: Minimized lattice parameters for Interface ......................................................... 55 


\section{List of abbreviations and symbols}

$\begin{array}{ll}\text { Abbreviation } & \text { Definition } \\ \text { HCP } & \text { Hexagonal closed packed } \\ \text { CANDU } & \text { CANada Deuterium Uranium } \\ \text { MD } & \text { Molecular Dynamics } \\ \text { 3D } & \text { Three Dimensional } \\ \text { EAM } & \text { Embedded Atom Method } \\ \text { MEAM } & \text { Modified Embedded Atom Method } \\ \text { LAMMPS } & \text { Large-scale Atomic/Molecular Massively Parallel Simulator } \\ \text { PBC } & \text { Periodic Boundary Conditions } \\ \text { RDF } & \text { Radial distribution function } \\ \text { MSD } & \text { Mean Square Displacement }\end{array}$

\section{Symbols}

$\sigma$

$\varepsilon$

E

v

B

D

K

$\AA$

ps

$\mathrm{GPa}$

$\mathrm{MPa}$

\section{Definition}

Stress

Strain

Young's Modulus

Poisson's ratio

Bulk Modulus

Diffusion coefficient

Kelvin

Angstroms

pico seconds

Giga Pascal

Mega Pascal 


\section{CHAPTER 1}

\section{INTRODUCTION}

\subsection{Motivation}

The behavior of zirconium and its alloys under various operating conditions in nuclear reactors has been studied extensively. Zirconium $(\mathrm{Zr})$ is a transition metal that has strongly anisotropic properties due to a hexagonal close packed crystal structure. It is extensively used in nuclear reactors as a structural material because of low thermal neutron absorption and good corrosion resistance even at high temperatures [5]. CANDU reactors and almost all other nuclear reactors have structural elements made of zirconium alloys [6]. In essence, zirconium alloys are the perfect choice for fuel cladding and pressure tubes in nuclear reactors.

The hydrogen generated during reactor operation has deleterious influence on mechanical properties of zirconium. The hexagonal close packed structure of zirconium has tetrahedral and octahedral sites and hydrogen generated in the reactor is expected to diffuse and occupy these sites [7]. At higher concentrations of hydrogen, zirconium hydrides are formed causing embrittlement. The hydrides are brittle and crack on application of stress, which reduces the performance and life expectancy of nuclear reactor thereby increasing the cost of nuclear power. As it is well known, hydride precipitates play an important role in the hydrogen embrittlement of various zirconium alloys, and a great number of studies [1-10] have focused on the deformation or fracture behavior of zirconium hydrides. Simpson et al. [1] performed fracture toughness tests under tension on specimens of bulk $\delta$-hydride and obtained an extremely low fracture toughness of $\sim 1 \mathrm{MPa} \mathrm{m}{ }^{1 / 2}$ at room temperature. Because hydride in $\mathrm{Zr}$ alloy specimens may contain defects such as voids or micro-cracks and there is often misfit stress between the hydride and zirconium matrix, the results obtained for pure hydride may not be directly applied to the hydride in the reactor.

Hydride reorientation [4] and cracking under hoop stress in zircaloy tubes is the major problem in the nuclear industry. The occurrence of hydride reorientation under stress usually involves the preferential precipitation of hydride along circumferential direction of zirconium tube. When this orientation is subjected to hoop stress the hydrides dissolves and precipitates along the direction normal to the applied stress that is along the radial direction. 
Szpunar et al [4] and others [27-30] have shown that hydride reorients on application of the hoop stress and this may result in zirconium tubes failure. Therefore, hydrides formation and reorientation have become a major issue in the nuclear industry and the study on hydride has gained significant importance in the nuclear research. Theoretical analysis of these processes was recently proposed by Qin et al [9].

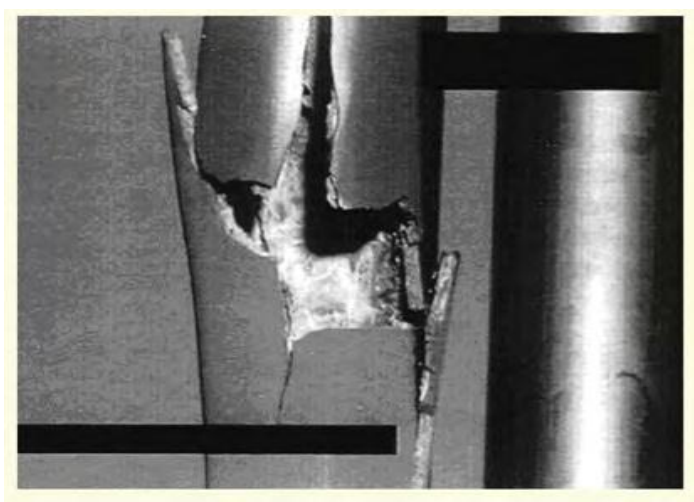

(A.N.T International)

Figure 1: Cracks caused due to hydrogen embrittlement in zirconium

However, the process of hydride reorientation requires further analysis of strain induced hydrogen diffusion and variation of energy of hydride structure under strain, such studies cannot be easily performed due to experimental limitations. In order to study these factors it is necessary to introduce atomic scale simulations by Molecular Dynamics (MD), this will allow us to understand formation of hydrides in zirconium and associated changes in mechanical properties.

\subsection{Research questions}

The fundamental question which motivates my thesis is: What is the process of hydride formation and reorientation in zirconium tubes used in nuclear reactors? To help answering this question, MD simulations are used. Using MD I am planning to answer the following questions:

1. Which is the best method that describes structure and mechanical properties of zirconium and zirconium hydride?

2. How temperature does affects yielding point in pure zirconium?

3. How does temperature affects diffusion of hydrogen and does strain effects hydrogen diffusion? 
4. What is the effect of stress and strain on orientation of hydride?

5. Determination of crack nucleation site at the interface of zirconium and zirconium hydride

6. How does hydrogen affect crack nucleation and propagation in zirconium?

\subsection{Organization of thesis}

Chapter 2 is a literature review of experimental results performed on zirconium and zirconium hydrides and Chapter 3 provides a general overview on molecular dynamics simulation, various potentials to be considered and software used in this research. Chapter 4 discusses results on verification of computer models and simulation methods performed on zirconium and zirconium hydride and comparisons made with experimental data. Chapter 5 we calculate stress and strain on zirconium using various simulation methods. Chapter 6 we study the effect of hydrogen diffusion in zirconium and the effect of strain on hydrogen diffusion. Chapter 7 provides explanation on effect of stress on hydride orientation. Chapter 8 discusses properties of the interface of zirconium and zirconium hydride. Chapter 9 explains the effect of hydrogen on crack propagation, using OVITO and VMD software. Chapter 10 summarizes results obtained, outlines limitation and propose continuation of this work. 


\section{CHAPTER 2}

\section{REVIEW OF EXPERIMENTAL RESULTS}

This chapter provides background information on experimental results on lattice parameters of zirconium, phase stability of zirconium hydride, grain boundaries, and preferential precipitation of hydrides in zirconium and effect of hoop stress on hydrides used in this research.

\subsection{Experimental lattice parameters of zirconium}

Zirconium crystallizes in hexagonal closed packed structure at room temperature and converts to body centered cubic above the temperature of $1143 \mathrm{~K}$.Lattice parameters for zirconium and variation of lattice parameters with temperature and thermal expansion are determined using $\mathrm{X}$ ray measurements $[17,18]$. Skinnert et al. [18] has seen the lattice parameters of pure zirconium at $25^{\circ} \mathrm{C}$ were found to be $\mathrm{a}=3.2265 \pm 0.0010 \mathrm{kX}$ and $\mathrm{c}=5.1371 \pm 0.0015 \mathrm{kX}$ and the average coefficient of linear thermal expansion between $298 \mathrm{~K}$ and $1143 \mathrm{~K}$, is $5.5^{*} 10^{-6} \mathrm{~K}^{-1}$ along the ' $\mathrm{a}$ ' axis and $10.8 * 10^{-6} \mathrm{~K}^{-1}$ along ' $\mathrm{c}$ ' axis. Goldak et al [17] fitted variation of lattice parameters of pure zirconium using least squares fitting method in the range of temperature range of (4.2 1125) $\mathrm{K}$ as shown Table.1.

Table 1: Variation of lattice parameter with temperature with zirconium

\begin{tabular}{|l|l|l|}
\hline Temperature $\left({ }^{\circ} \mathrm{K}\right)$ & Lattice parameter ' $\mathrm{a}$ ' $(\AA)$ & Lattice parameter 'c' $(\AA)$ \\
\hline 4.2 & 3.2294 & 5.1414 \\
\hline 300 & 3.2331 & 5.1491 \\
\hline 800 & 3.24146 & 5.1737 \\
\hline 1125 & 3.2468 & 5.1964 \\
\hline
\end{tabular}

\subsection{Structure of hydride}

Zirconium hydrides have face centered crystal structure with zirconium atoms at the face centered sites and hydrogen atoms occupying tetrahedral sites [7, 13]. There are three hydride phases with composition of $\mathrm{ZrH}(\gamma)$ tetragonal, $\mathrm{ZrH}_{1.6-1.7}(\delta)$ cubic and $\mathrm{ZrH}_{1.74-2}(\varepsilon)$ tetragonal or 
$\mathrm{ZrH}_{2}$ that are formed by varying the hydrogen concentration of the material. There is some discrepancy in results on formation of hydride at higher concentrations of hydrogen i.e. $\varepsilon$-face centered tetragonal and $\delta$-face centered cubic in the literature [13, 20-23]. For hydrides precipitating in $\alpha$-zirconium matrix, the literature is full of seemingly contradictory results as to what conditions favor the formation of a given hydride phase. Face centered tetragonal ( $\varepsilon$ - fct) zirconium hydride is a metastable state that is formed from face centered cubic $(\delta$-fcc). The formation of tetragonal distortion is observed in ab -intio calculations $[12,14]$ and it is said to be related to splits of orbitally degenerate states with a peak in the density of states at the Fermi energy, similarly as claimed by Switendick for $\gamma$ phase distortion [24]. Also Paolucci et al. [25] suggested that the driving force for the $\varepsilon$ - fcc to $\delta$-fcc phase transition is the decrease in the density of states at the Fermi level $\left(\mathrm{E}_{\mathrm{F}}\right)$ with a small shift of $\mathrm{E}_{\mathrm{F}}$ to lower energy.

\subsection{Grain boundaries}

Grain boundaries have long been recognized as important microstructural elements in engineering materials. They play a key role in determining the properties of materials, especially when grain size decreases and even more so with the current improvements of processing tools and methods that allow us to control various structural elements of polycrystalline materials. Grain boundaries represent the simplest interfaces. The grain boundary is represented by five independent parameters which provide us with information on misorientation between two grains and the orientation of plane separating these grains. Three of them specify misorientation of the adjoining grains A and B as shown in figure. 2. This misorientation is represented by a rotation, which brings both grains into the same orientation.

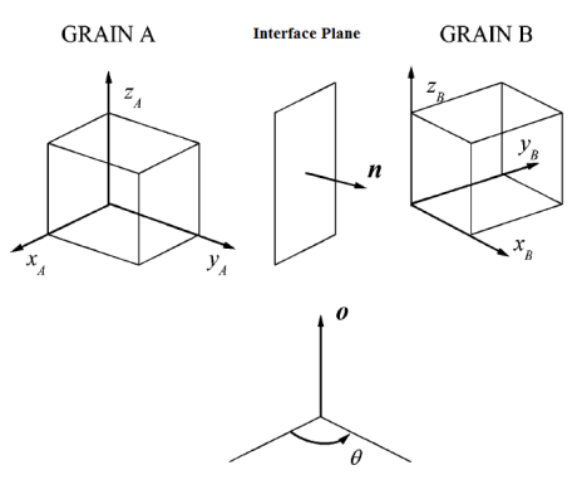

Figure 2: Variables that define a grain boundary $\mathrm{xA} ; \mathrm{yA} ; \mathrm{zA}$ and $\mathrm{xB}, \mathrm{yB}, \mathrm{zB}$ are the axes of the co-ordinates parallel to crystallographic directions in grains $\mathrm{A}$ and $\mathrm{B}$, respectively. $\mathrm{O}$ is the 
rotation axis and $\theta$ is the rotation (misorientation) the angle necessary to rotate one grain to orientation of another $n$ determines the orientation of the grain boundary plane. From Lejcek [26]

\subsection{Preferential precipitation of hydrides}

Recent studies on hydrides using optical microscopy (OM) and electron backscatter diffraction (EBSD) technique by K.Kiran et al [8] and other researchers [27-30] concluded that $\delta-\mathrm{ZrH}_{1.5}$ phase were located more often at $\mathrm{Zr}$ grain boundaries than in intra-granular area. These hydrides formed at both inter-granular and intra-granular regions follow the orientation relationship of $(0$ $\left.\begin{array}{lll}0 & 0 & 1\end{array}\right) \alpha-\mathrm{Zr} / /\left\{\begin{array}{lll}1 & 1 & 1\end{array}\right\} \delta-\mathrm{ZrH}_{1.5}$ with the zirconium matrix. Figure 3 shows EBSD studies on preferential hydride, pole figures shows hydride being marked as $\{111\}$ plane with (0001) of $\alpha-Z r$. The orientation relationship remains the same even after changing the hydrogen content. These studies are of importance as they provide us information on hydride orientation in zirconium matrix and this orientation relationship is used in this research to study the properties of interface and their failure.

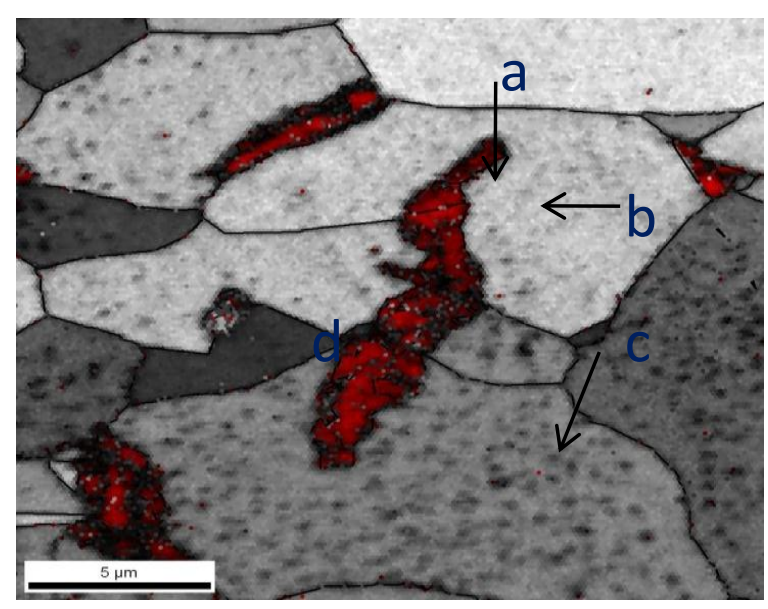

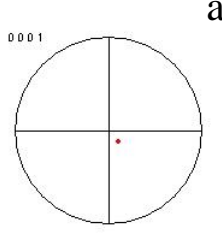

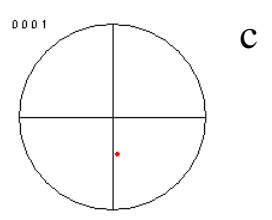

C

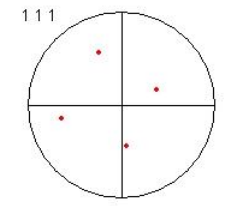

Figure 3: EBSD image of preferential precipitation of hydrides at grain boundary, orientation relation illustrated by pole figures from N.A.P Kiran Kumar et al [8]

\subsection{Reorientation of zirconium hydrides under stress}

The hydrides often form platelets and the orientation of these platelets towards radial direction adversely affects the mechanical properties of the fuel cladding. Factors such as temperature gradient, stress gradient, texture and the fabrication method play an important role in the formation of radial hydrides in $\mathrm{Zr}$ alloys. The radial hydride formation is believed to be driven 
by hoop stress, which acts as a tensile stress along the circumferential direction. The significance of the stress-reoriented hydrides became apparent when the failure of an in-reactor fuel element is analyzed. It was demonstrated that even a sample with $40 \mathrm{ppm}$ of hydrogen can experience brittle failure, if the hydrides platelets are precipitated perpendicular to the tensile stress direction. K. Kumar et al [8] have shown that preferential formation of hydride i.e. unstressed alloy always forms (0001) $\alpha-\mathrm{Zr} / /\{111\} \delta-\mathrm{ZrH}_{1.5}$ orientation as shown in figure 4(a) and when hoop stress $(\sigma)$ is applied the hydride dissociates, dissolves, reprecipitates and reorients in $\{10$ $\overline{1} 1\} \alpha-\mathrm{Zr} / /\{111\} \delta-\mathrm{ZrH}_{1.5}$ as shown in figure 4(b). Colas et al. [28] studied using synchrotron radiation in situ the kinetics of hydride dissolution and precipitation in previously hydride zircaloy samples. Wen et al [9] in his theoretical model claimed that the reorientation is mainly due to change in grain boundary energy caused by stress. In this regard, Hong et al [29] has shown that hydride reorientation in a stressed sample takes place along radial direction of the tube when cooled from $300^{\circ} \mathrm{C}$ to $200^{\circ} \mathrm{C}$. Hong further proposed time dependent stress-aided dissolution of circumferential hydrides and reprecipitation of radial hydrides. Chu et al [30] has considered thermal cycling as an important factor and claimed that threshold stress for hydride reorientation depends on hydrogen concentration and proposed that diffusion of hydrogen can play a major role in the reorientation process. It was made clear by these works that stress plays a major role in hydride reorientation.
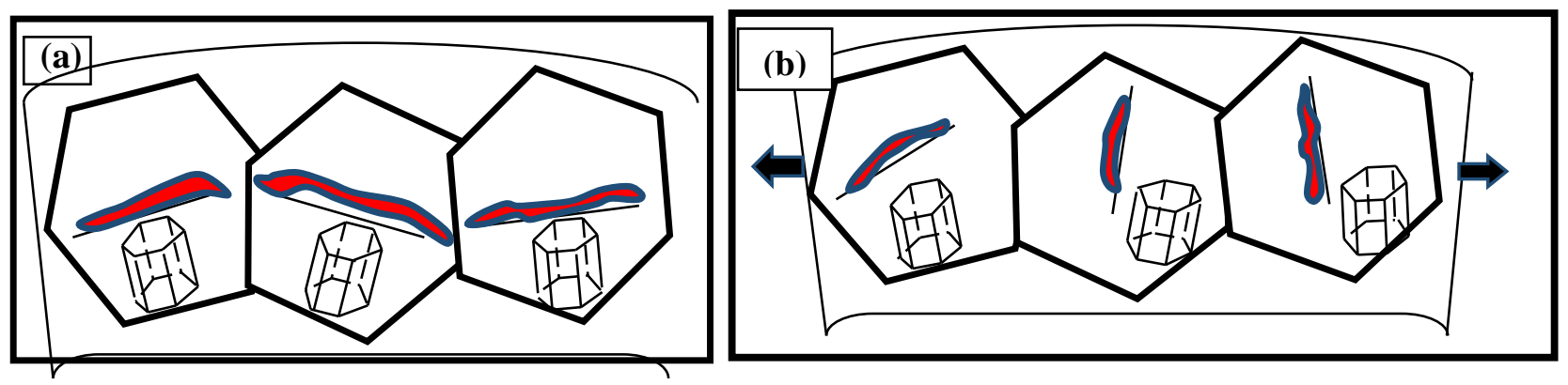

Figure 4 : Schematic view of a hydride orientation in cladding a) without hoop stress and b) under hoop stress

In summary, despite knowing deleterious influence of radial hydrides, very little information is available on crystallographic orientation of hydrides and the behavior of reoriented hydrides and more work on the role of applied stress in hydrogen diffusion need to be done Therefore, systematic investigations using molecular dynamics can give us better understanding of processes related to formation of radial hydrides. 


\section{CHAPTER 3}

\section{MOLECULAR DYNAMICS}

Computer simulations play an important role in science of today. They act as a bridge between theory and experiments [31]. Molecular dynamics (MD) is a computer simulation technique where the time evolution of a set of interacting atoms in a crystal, followed by integrating their equations of motion. In molecular dynamics, especially in metal atoms interact with each other generating forces between them known to be short range interactions. These interactions generate forces relative to their positions [32]. Classical Newton's laws of motion are then applied to compute positions of atoms and their velocities [31, 32]. The force acting on each atom $i$ in a system having an atomic mass $m$ is given by eq. (1),

$$
\vec{F}=\vec{m} * \vec{a}
$$

The computer calculates a vectors in a $6 \mathrm{~N}$-dimensional phase space $(3 \mathrm{~N}$ positions and $3 \mathrm{~N}$ velocities). A trajectory obtained by molecular dynamics provides a set of configurations by simulation of an arithmetic average of the various instantaneous values assumed by that quantity during the MD run.

\subsection{Molecular Dynamics Flowchart}

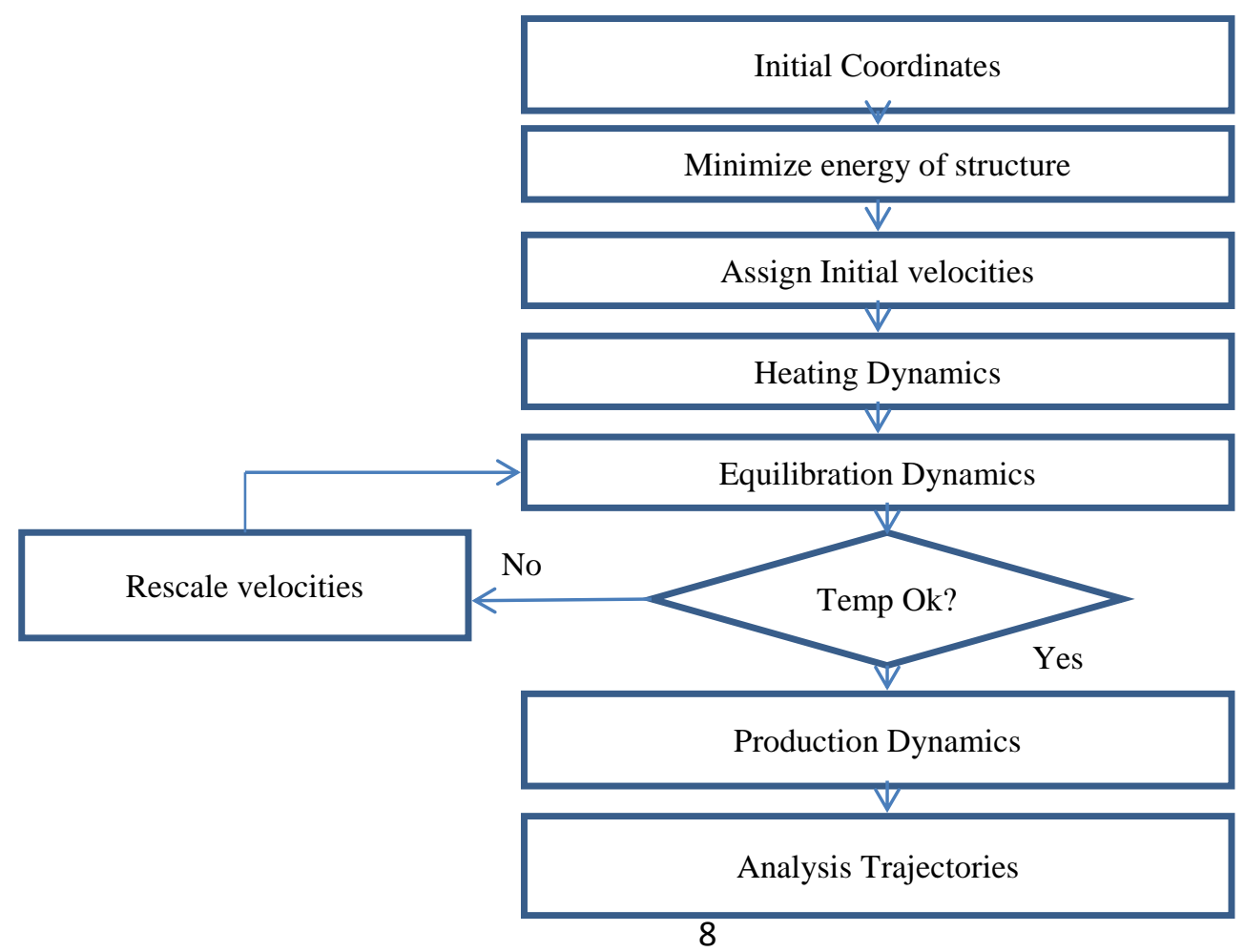


The above flow chart shows the general procedure followed in molecular dynamics. Initially, the atomic coordinates corresponding to the crystal structure is assigned. The geometry of the structure is optimized by minimizing the energy of the structure so that the final configuration of the structure is in equilibrium state with zero velocities. This state is known as minimum energy state and gives us the lattice parameters corresponding to the potential. Then, the atoms are assigned velocities so that the vibration of these atoms generates the required temperature in the system. The system is allowed to stabilize at this temperature. If the required temperature in the structure is not obtained or if temperature is highly fluctuating the velocity rescale is applied until the required temperature is reached. Then dynamics of various process parameters related to strain, deformation, temperature, or pressure are applied on the system to obtain trajectories at various time steps.

\subsection{Boundary Conditions}

Boundary conditions are one of the important factors to be considered in molecular dynamics simulation. There are four possible boundary conditions [33]:

- Periodic boundary condition (p) - means the simulation box is periodic, so that particles interact across the boundary, and they can exit one end of the box and re-enter the other end as shown in figure 5 .

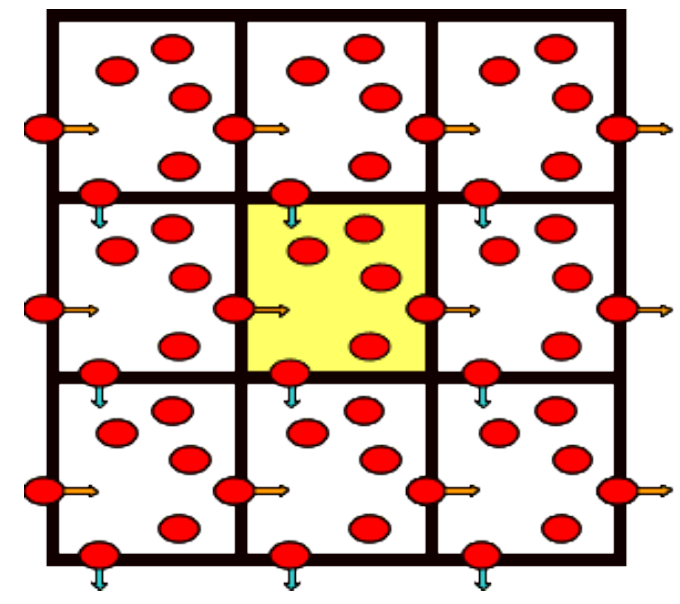

Figure 5: Periodic boundary conditions (PBC) is where atoms leave from one box and enter into another box keeping the total number of atoms constant and the area shaded yellow is the area of interest. 
- Free surface, rigid and flexible boundary conditions [39] means that atoms cannot enter the simulation box or leave it for example a surface simulation. The rigid condition allows the atoms or side of simulation be fixed. Flexible boundary allows small displacements of atoms in response to forces exerted on the cell at the perimeter of the computational cell. Different types of boundary conditions are shown in figure 6(a), (b) and (c).

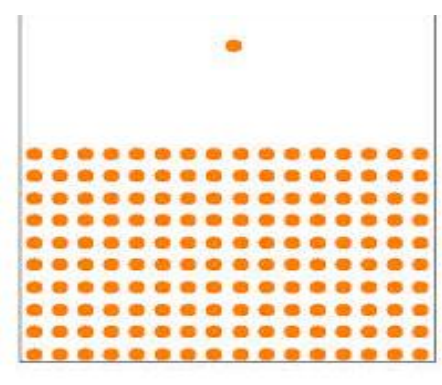

(a)

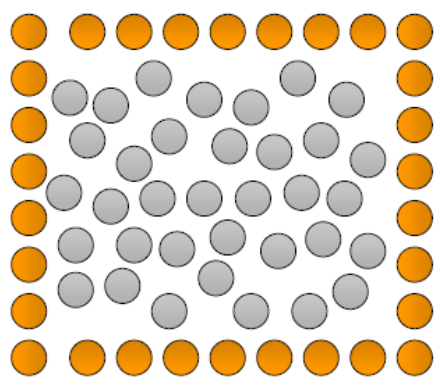

(b)

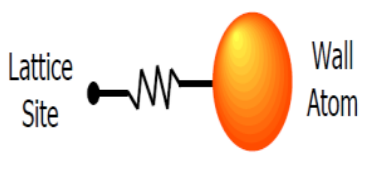

(c)

Figure 6: Boundary conditions that can be used in molecular dynamics. (a) Free surface boundary condition where atoms leave the surface. (b) Rigid surface where atoms shown in yellow act as a wall and do not move with time used for simulating fluid mechanics. (c) Flexible boundary condition are used to make boundaries rigid enough to maintain structure and flexible enough to interact with the other atoms.

\section{Thermodynamic ensembles}

NPT (Constant pressure and Temperature)

NPT ensemble allows control over both the temperature and pressure. The unit cell volume is allowed to change by keeping the pressure and temperature constant. This ensemble can also be used during equilibration to achieve the desired temperature and pressure.

NVT (Constant Volume and Temperature)

NVT ensemble obtained by controlling the temperature through direct temperature scaling. The volume is kept constant throughout the run. This ensemble is mostly used during production runs allowing to collect data like displacement of atoms at constant temperature.

NVE Ensemble (Constant Volume and Energy)

Total energy is conserved when this ensemble is initialized. However, because of rounding and truncation errors during the integration process, there is always a slight drift in energy. 


\subsection{Significance of potentials in Molecular Dynamics}

Molecular dynamics simulations depends on choosing the right potential: a function of the positions of the nuclei, representing the potential energy of the system when the atoms are arranged in that specific configuration was described earlier. This function is translationally and rotationally invariant, and is usually constructed from the relative positions of the atoms with respect to each other, rather than from the absolute positions. In metals, strong interatomic bond formed between two atoms is mainly due to metallic bonding which generates forces between atoms. They originate from the free valency electron 'sea', which holds the ionic core. Thus a strong exponentially attractive force is observed which is known to be a potential function. Potential function [31] ' $V$ ' between two atoms $i$ and $j$ interacting with each other at a distance $r$ in a system of $\mathrm{N}$ atoms is given as sum of pairwise interactions written as

$$
V\left(r_{1}, \ldots \ldots . r_{N}\right)=\sum_{i} \sum_{j>i} \Phi\left(\left|r_{i}-r_{j}\right|\right)
$$

In Eq. (2), ' $\phi$ ' is a pair-potential which is a function of the scalar distance between atoms $i$ and $j$. The basic interaction between the nuclei is modeled as a pair-potential e.g. Coulomb electrostatic interaction, Lennard-Jones potential interaction or a Morse potential interaction. However in order to describe behavior of atoms in metals we require potential which describes interactions between nuclei and also nuclei with surrounding valence electrons. Commonly used potentials for engineering applications in metals are the embedded atom method (EAM) and Modified embedded atom method (MEAM).

\subsection{Embedded Atom Method (EAM)}

EAM is an inter-atomic potential developed by Daw et al [34] for metals. This method is one of the most accurate for crystal metal lattices. It has been applied to date to a variety of problems in pure metals and alloys. A single crystal metal lattice consists of positively charged nuclei embedded in a 'sea' of valence electrons that binds the nuclei together. Since the arrangement of the nuclei is periodic in all directions in the metal lattice, the inter-metallic bond is nondirectional [33], so the total energy of the system is evaluated by two factors; one is the interaction of every atom with local electron density of the entire system of atoms called 
embedding energy and second is pairwise interactions created by electrostatic interactions between atoms. It can be written in the form of equation.

$$
E_{c o h}=\sum_{i} G_{i}\left(\sum_{j \neq i} \rho_{j}^{a}\left(R_{i j}\right)\right)+\frac{1}{2} \sum_{i, j(j \neq i)} U_{i j}\left(R_{i j}\right)
$$

In Eq. (5), $G$ is the embedding function, $\rho^{\mathrm{a}}$ is the spherically averaged atomic electron density and $U$ is an electrostatic, two-atom interaction $i$ and $j$ at a distance $R$.

The main disadvantage of EAM potential is that it does not incorporate angular dependency [35] which is required for diatomic gaseous elements and therefore is suitable only for metallic crystal structures. In order to overcome this problem modified embedded atom (MEAM) was developed.

\subsection{Modified Embedded Atom Method (MEAM)}

MEAM developed by Baskes [35] is the first interatomic potential formalism that has the possibility of describing a wide range of elements like fcc, bcc, hcp, diamond-structured and even gaseous elements using the same method. MEAM is created as an extension to EAM with the addition of directional bonding. The potential MEAM used in this research was developed by B. Szpunar et al [12]

However in order to calculate forces on each atom we require powerful molecular dynamic software like Large-scale Atomic/Molecular Massively Parallel Simulator (LAMMPS) which can support different potentials.

\subsection{Lattice constants}

Minimized lattice constants enables one to determine the zero-temperature, relaxed, nonvibration structure of the system by minimization of energy [39]. It has been widely used in problems dealing with low-temperature, crystallographic structure, high temperature, nonvibration structure and energetic defects in liquid, amorphous and crystalline state. If desired configuration of system is in equilibrium then the force on any atom i must vanish, this condition is a basis of any calculation as shown in equation 6 . 


$$
F_{i}=-\frac{\partial V}{\partial r_{i}}=-\sum_{j \neq i} \frac{\partial V_{j}\left(r_{i j}\right)}{\partial r_{i j}}
$$

In calculation of lattice constants, energy minimization requires that that each atom moves in the direction of the force acting on it by a certain amount. This amount can be governed by the force acting on the atom and the existing interaction with other atoms. This process is repeated until all forces are reduced essentially to zero, i.e., below some numerical value. The system is then considered "relaxed". The configuration obtained and the energy of the system at $\mathrm{T}=0$, is the simplest since at zero temperature the higher order terms of energy vanish.

\subsection{Cohesive Energy}

The cohesive energy is the difference between the energy per atom of the bulk material at equilibrium and the energy of a free atom in its ground state [39]. Cohesive energy is of fundamental importance in the theory of metals since it is the basis of understanding properties such as the structures, elastic constants and stacking faults.

\subsection{Molecular Dynamics Simulation software}

In the present research computational resources available in Westgrid were used. Westgrid is a part of Compute Canada which provides high performance computer resources. Westgrid provides terabytes of data storage and offers 4328 cores of processors. It has various software which are used in this research such as, LAMMPS [36], OVITO [37] and VMD [38]. The access to this resource was provided by my supervisor. LAMMPS is molecular dynamics software which can run on single and multiple processors. It contains and supports various potentials. The files obtained from LAMMPS for visualization of deformation and cracking can be illustrated by using software like OVITO and VMD. The results obtained are evaluated by comparing them with the experimental data. Potentials such as EAM for zirconium and MEAM potential for zirconium hydride were available from the literature.

\subsubsection{LAMMPS: - large-scale atomic/molecular massively parallel simulator}

The program used to run the molecular dynamics simulations is LAMMPS. This software is most powerful MD software designed by Sandia National Laboratories, USA. LAMMPS is aimed for molecular dynamics which are governed by Newton's equations of motion. LAMMPS 
has potentials for soft materials like (biomolecules, polymers) and solid-state materials (metals, semiconductors). This program enables us to perform simulations on computers with multiple processors in ways that significantly decreases the simulation time as the number of processors is increased.

A code file is needed to define the parameters for the material and simulation environment, and then give instructions on the type of simulated experiment to perform. An important factor in molecular dynamics simulations is the time step $(\Delta \mathrm{t})$. The time step is the amount of time between each calculation interval in which the force applied to each atom and velocities are calculated. The time steps are repeated $t_{i+1}-t_{i}=\Delta t$ until the chosen number of iterations is completed. In order to capture the fast motion of the atoms, a femtosecond $\left(10^{15}\right)$ time step is required. The procedure for writing an input script in LAMMPS is given through the following steps.

\section{Steps Followed to perform MD using LAMMPS}

The flowchart below shows the steps to be performed in LAMMPS in order to run MD and the description of each step is given below

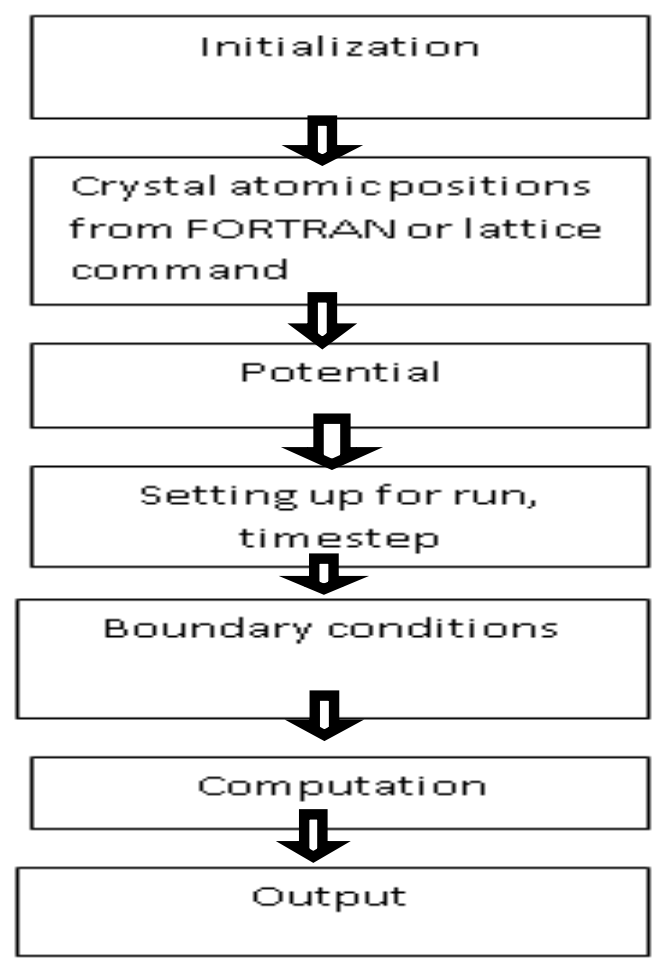


Step 1:-Initialization

At the initial stage atomic positions and their respective lattice parameters are defined either by using FORTRAN program or by defining the lattice. LAMMPS contains various lattice structures such as fcc, hcp, square, diamond and also customized lattice parameters can be defined for complicated structures. The most common crystal structures and their lattice parameters can be obtained from literature.

\section{Step 2:-Potential}

The potential defines the type of forces that are acting on neighboring atoms. The potential file must be defined properly so that the types of atoms are correctly assigned. Different potentials require different types of files to define the interaction between the atoms. LAMMPS contains various potentials for various elements and they can be accessed in their website.

Step 3:- Run and Time step

Timestep command sets time step size for subsequent molecular dynamics simulations. Run command continues dynamics for a specified number of time steps.

Step 4: Boundary Conditions

The type of boundary conditions signifies the type of neighbors an atom possesses. A periodic boundary condition defines same neighborhood for inner as well as outer atoms in all dimensions. A non-periodic in a particular dimension varies the type of neighborhood in that particular dimension.

\section{Step 4:-Computation}

Computation command calculates various physical quantities like temperature, pressure mean square displacement and other parameters for a defined group of atoms. Quantities calculated by this command are instantaneous values i.e. the current information about a certain quantity is based on previously stored data. The computation obtained can be both for global or local group of atoms as defined in the program. 
Step 5:-Output or Post-processing

The output obtained from LAMMPS is used to calculate various values such as lattice parameters, Young's modulus, bulk modulus, Poisson's ratio, cohesive energy, and stressstrain. Microsoft Excel is used to plot graphs and to obtain parameters for calculation of lattice parameters change with temperature. Also the output can be visualized through OVITO and VMD.

\subsubsection{OVITO}

The output or dump from LAMMPS is visualized using Ovito software. OVITO is a scientific data visualization and analysis software for large-scale atomistic simulations, in particular classical molecular dynamics (MD) [37]. OVITO is freely available under an opensource license for Windows, Linux and MacOS platforms. It is being developed by Alexander Stukowski at the Material Science Department at Darmstadt University of Technology, Germany. The type of analysis apart from visualization is the Bond Angle Analysis [58] and Common Neighbor Analysis [58]. The Bond Angle Analysis which gives the type of bond formed between two local neighbor atoms using Auckland-Jones bond angle method [58] and also Common Neighbor Analysis (CNA) which shows type of crystal structure environment like FCC, BCC, and HCP etc.

\subsubsection{VMD}

Visual Molecular Dynamics is another type of software designed to visualize dump or output files from LAMMPS. This software is useful in visualizing the stresses surrounding each atom. VMD can also be used to animate and analyze the trajectory of a molecular dynamics (MD) simulation. In particular, VMD can act as a graphical front end for an external MD program by displaying and animating a molecule undergoing simulation on a remote computer.

\subsection{Summary}

The atomistic simulation methods at the atomistic and microscopic levels have an intimate interrelation that can be exploited in an integrated approach to modeling complex systems 
such as interfaces. Such a complex task requires fast computational capabilities, to enables one to take advantage of the unique capabilities of each of the two atomistic methods (EAM and MEAM) in a systematic investigation of structure property correlations. These correlations can be studied from zero temperature to a very high temperature, and can include the effect of stress applied to the system. The goal of this work is to optimize the potential for zirconium and zirconium hydride through various calculations and then verifying the results with experimental data. This optimized potential is then used to study complicated engineering problems like effect of hydrogen on strength of zirconium, effect of hydrogen in crack propagation at various temperatures, properties of hydride at the interfaces with zirconium and effect of stress on diffusion of hydrogen in zirconium matrix. 


\section{RESULTS AND DISCUSSION}

\section{CHAPTER 4}

\section{VERIFICATION COMPUTER MODELS AND SIMULATION METHODS}

As described in the chapter 3, an integrated computer simulation of hydride reorientation and interfacial cracking requires a set of computer models to calculate the interactions between atoms and to describe the motion of atoms and to relate this motion to macroscopic quantities that are to be computed. The purpose of this chapter to verify potentials for zirconium and zirconium hydride by comparison with experimental data as well as other theoretical data in order establish the best potential. The comparison is done between EAM and MEAM potentials through calculating lattice parameters, cohesive energies, stiffness constants and mechanical properties.

\subsection{Structure of zirconium}

Zirconium as we know has HCP structure with different ' $a$ ' and ' $c$ ' lattice parameters where atoms are in arranged in $\mathrm{ABABAB} \ldots$ stacking sequence. Lattice parameters are calculated using the EAM and MEAM potentials in order to propose the best potential for zirconium metal. A structure of zirconium is created assuming periodic boundary conditions in all three directions as shown in figure 7 (a) and (b).
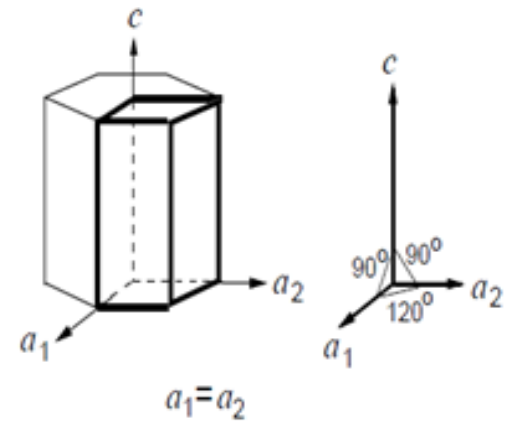

Figure 7: (a) Unit cell HCP.

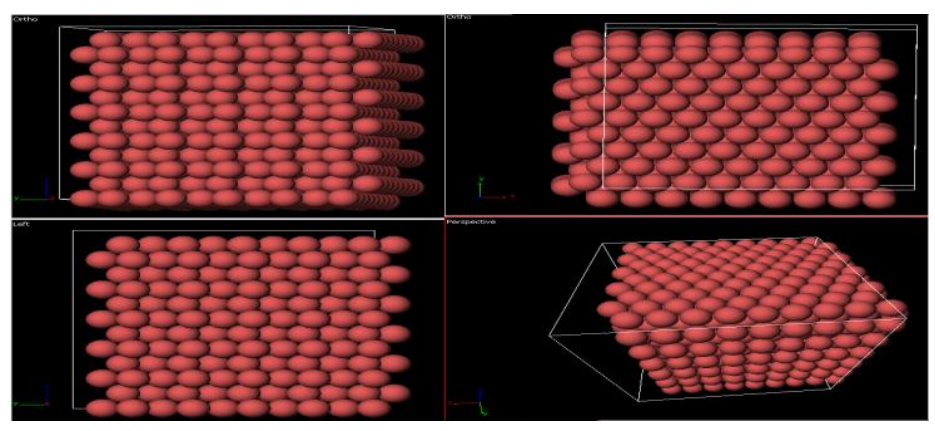

(b) Atomic arrangement of $\mathrm{Zr}$

\subsubsection{Lattice parameters for zirconium using EAM and MEAM potentials}

The lattice parameters are evaluated when the energy of the system is at its minimum. The total energy of the system is its potential energy. An external pressure or stress tensor is 
applied to the simulation box during energy minimization. This allows the box size and shape to vary during the iterations of the minimizer so that the final configuration of structure will correspond to potential energy minimum and the system pressure tensor will be close to the specific external tensor. The volume and energy convergences of EAM for the HCP zirconium structure are shown in Figure 8(a) and (b) respectively. Figure 9(a) and (b) shows energy convergence of MEAM potential. The Lattice parameters and the cohesive energy are calculated from this minimum energy state are compared with the experiments and shown in table 2.
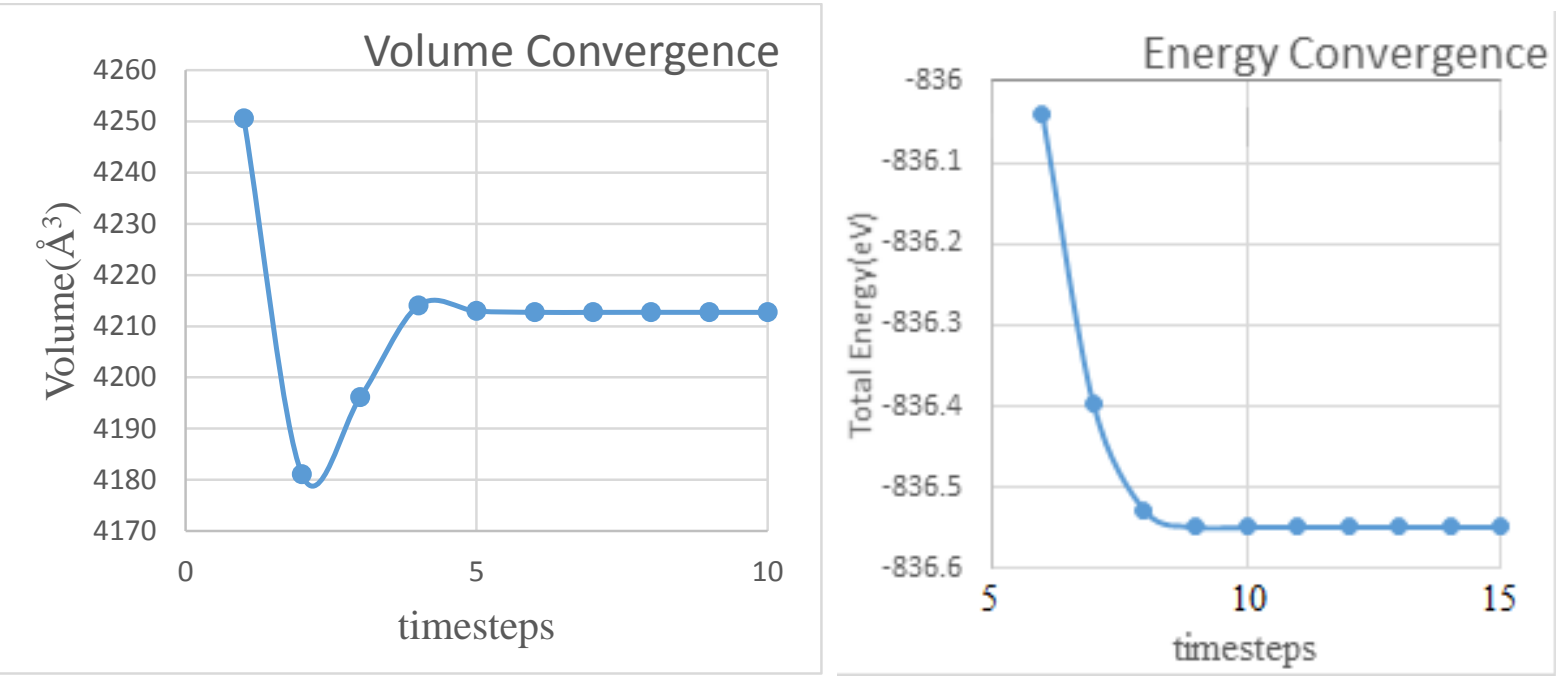

Figure 8: Convergence of EAM potential (a) Volume convergence (b) Energy convergence.
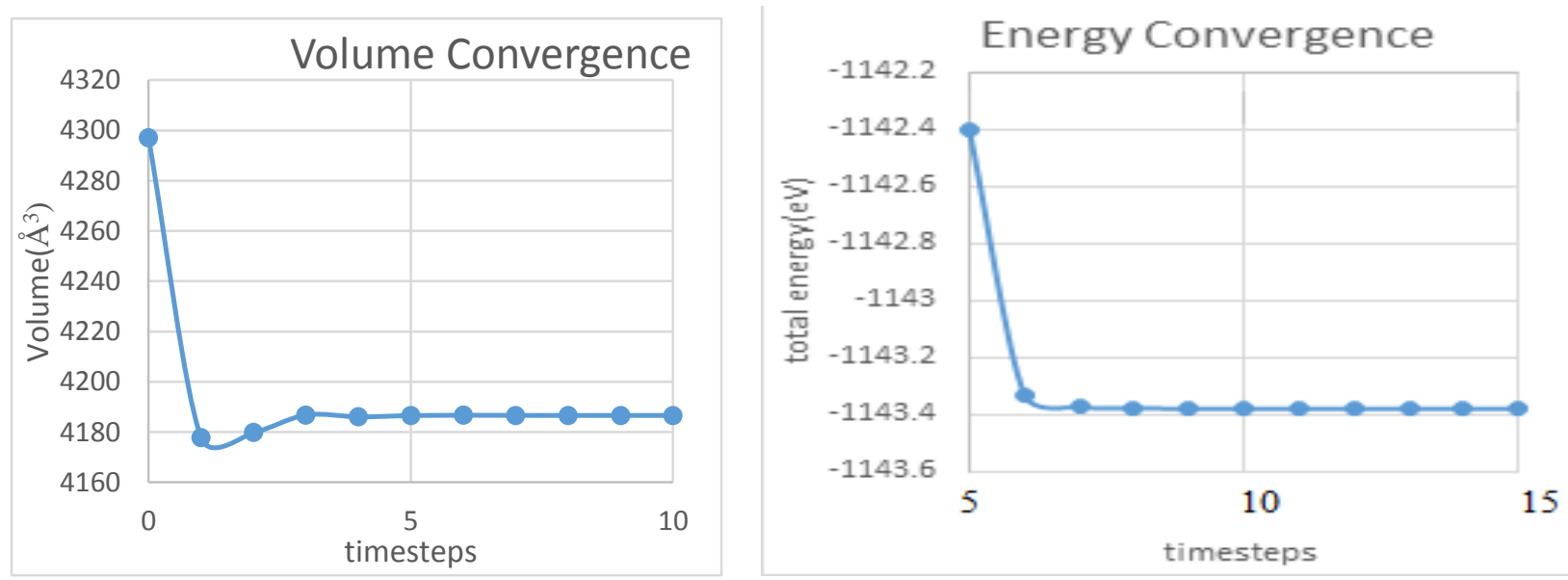

Figure 9: Convergence of MEAM potential (a) Volume convergence (b) Energy convergence

From the above stable state lattice parameters, cohesive energy and the total energy are obtained as shown in Table 2. 
Table 2: Lattice parameters and cohesive energy for zirconium calculated using EAM and MEAM potentials in comparison with experimental values

\begin{tabular}{|l|l|l|l|l|}
\hline \multicolumn{1}{|c|}{ Parameters } & \multicolumn{1}{|c|}{ EAM } & MEAM & $\begin{array}{l}\text { Other } \\
\text { Simulated } \\
\text { works }^{[11]}\end{array}$ & $\begin{array}{l}\text { Experimental } \\
\text { values }\end{array}$ \\
\hline $\mathrm{a}(\AA)$ & 3.2340 & 3.2165 & 3.2340 & $3.232^{[40]}, 3.2331^{[41]}, 3.231^{[48]}$ \\
\hline $\mathrm{c}(\AA)$ & 5.1676 & 5.1916 & 5.1676 & $5.182^{[40]}, 5.14905^{[41]}, 5.148^{[48]}$ \\
\hline $\mathrm{c} / \mathrm{a}$ & 1.5978 & 1.6140 & 1.5978 & $1.6033^{[40]}, 1.5931^{[48]}$ \\
\hline $\begin{array}{l}\text { Cohesive Energy } \\
(\mathrm{eV} / \text { atom })\end{array}$ & -4.647 & -6.352 & -6.6347 & $-6.32^{[40]},-6.36^{[48]}$ \\
\hline Total Energy $(\mathrm{eV})$ & -4182.742 & -5716.898 & -4776.990 & - \\
& & & & \\
\hline
\end{tabular}

The above table presents calculated lattice parameters in 'a' and 'c' crystal directions of zirconium. These values are in a good agreement with the experimental data. MEAM shows lower total energy in compared to EAM. Both MEAM and EAM show good agreement of 'c/a' with the experimental data. These values indicate stable structure of zirconium at room temperature.

\subsubsection{Stiffness constants of zirconium}

The minimum energy structure calculation is then used to verify stiffness of zirconium and to compare it with experimental data. The structure is subjected to positive (tensile) and negative displacements (compressive) and the resultant of the two is calculated for one row of the stiffness matrix. This process is repeated for 3-D structure in order to obtain stiffness/ elastic constants in different directions as zirconium is anisotropic in nature. The code used here is developed by Adian Thompson of Sandia Labs [36]. The stress and strain notation for a cubic cell is shown in figure 11(a) and (b) Stiffness values calculated using EAM and MEAM for HCP zirconium structure are given in Table 3. 


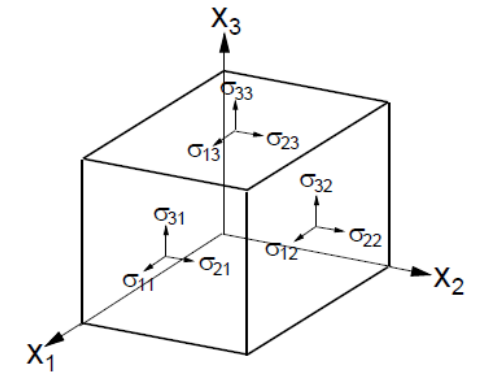

(a)

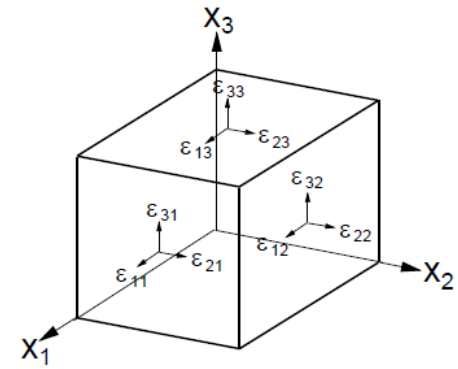

(b)

Figure 10: (a) and (b) Stress and strain directions on cubic crystal in different directions

Table 3 Stiffness values for $\alpha-Z r$ using EAM, MEAM, and comparison with experimental values

\begin{tabular}{|l|l|l|l|l|}
\hline Stiffness values & EAM & MEAM & Literature & Experimental values \\
& & & & \\
\hline $\mathrm{C}_{11}(\mathrm{GPa})$ & 141.59 & 135.27 & $147^{[1]}$ & 153 \\
\hline $\mathrm{C}_{12}(\mathrm{GPa})$ & 74.27 & 88.86 & $69^{[1]}$ & 67 \\
\hline $\mathrm{C}_{13}(\mathrm{GPa})$ & 74.08 & 63.12 & $74^{[1]}$ & 65 \\
\hline $\mathrm{C}_{33}(\mathrm{GPa})$ & 167.72 & 170.1 & $168^{[1]}$ & 172 \\
\hline $\mathrm{C}_{44}(\mathrm{GPa})$ & 43.93 & 18.79 & $44^{[1]}$ & 36 \\
\hline Bulk Modulus $(\mathrm{GPa})$ & 93.61 & 93.30 & $96.8^{[2]}$ & 96.7 \\
\hline
\end{tabular}

The stiffness properties using EAM and MEAM potential for zirconium shows good agreement with experimental data. However MEAM shows lower stiffness along $\mathrm{C}_{44}$ direction indicating weaker interactions of atoms along $\mathrm{C}_{44}$ direction. However, the bulk modulus calculated is in good agreement with experimental data showing the potentials.

\subsubsection{Comparison of Young's Modulus and Poisson's ratio for EAM, MEAM}

The Young's Modulus, 'E' is a material property that describes its stiffness and is therefore one of the most important properties in engineering design. The Young's Modulus of the material is calculated until the elastic limit i.e. until stress is directly proportional to stain. It has wide application in engineering design of materials. The Young's modulus is calculated using the stiffness matrix which is previously calculated. As zirconium is anisotropic the Young's modulus 
varies with directions of crystal structure. These values are calculated for the minimized structure and are compared with available experimental values.

The Matrix below shows a) stiffness and b) Compliance

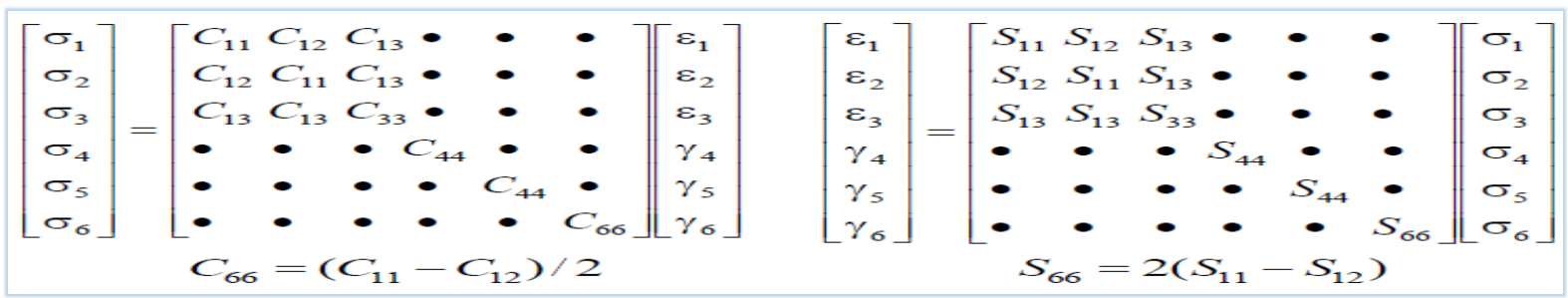
a) Stiffness Matrix
b) Compliance matrix

The calculation of Young's Modulus using stiffness matrix is calculated as shown below

$$
\begin{array}{lll}
\sigma_{1}:\left(\sigma_{2}=\sigma_{3}=0\right): & E_{(2 \overline{1} \overline{1} 0)}=\sigma_{1} / \varepsilon_{1}=\left(S_{11}\right)^{-1} & \varepsilon_{2}=S_{12} \sigma_{1}, \varepsilon_{3}=S_{13} \sigma_{1} \\
\sigma_{2}:\left(\sigma_{1}=\sigma_{3}=0\right): & E_{(01 \overline{1} 0)}=\sigma_{2} / \varepsilon_{2}=\left(S_{11}\right)^{-1} & \varepsilon_{1}=S_{12} \sigma_{2}, \varepsilon_{3}=S_{13} \sigma_{2} \\
\sigma_{3}:\left(\sigma_{1}=\sigma_{2}=0\right): & E_{(0001)}=\sigma_{3} / \varepsilon_{3}=\left(S_{33}\right)^{-1} & \varepsilon_{1}=S_{13} \sigma_{3}, \varepsilon_{2}=S_{13} \sigma_{3}
\end{array}
$$

\section{$\underline{\text { Poisson's Ratio }}$}

Poisson's ratio is another important physical property of a material. Poisson's ratio is the ratio of the relative contraction strain, or transverse strain normal to the applied load, to the relative extension strain, namely axial strain in the direction of the applied load. Poisson's ratio helps us to understand this theory. The Poisson's ratio varies for HCP zirconium structure as the Young's modulus varies as shown in figure 11. It is calculated for different directions according to the equation 7 .

$$
v_{1}=\frac{-\left(\varepsilon_{2}+\varepsilon_{3}\right)}{2 \varepsilon_{1}}=\frac{-\left(S_{12}+S_{13}\right)}{2 S_{11}} ; \quad v_{2}=\frac{-\left(\varepsilon_{1}+\varepsilon_{3}\right)}{2 \varepsilon_{2}}=\frac{-\left(S_{12}+S_{13}\right)}{2 S_{11}} ; \quad v_{3}=\frac{-\left(\varepsilon_{2}+\varepsilon_{1}\right)}{2 \varepsilon_{3}}=\frac{-\left(S_{13}+S_{13}\right)}{2 S_{33}}
$$

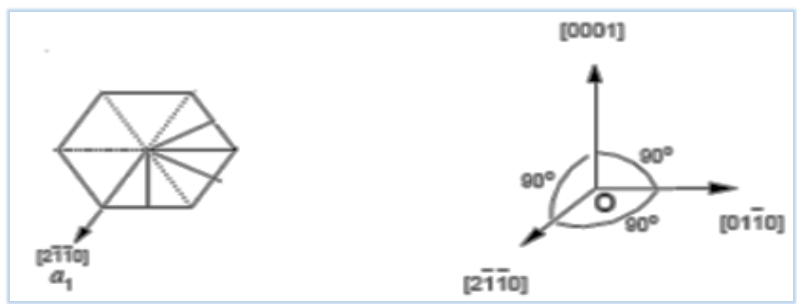

$\mathrm{Eq}(7)$

Figure 11: Directions of hexagonal crystal in which anisotropy is calculated 
Table 4: Young's Modulus and Poisson ratio in comparison with experimental values

\begin{tabular}{|c|c|c|c|}
\hline Young's Modulus & EAM & MEAM & Experimental values ${ }^{[42}$ \\
\hline$\left[\begin{array}{llll}2 & \overline{1} & \overline{1} & 0\end{array}\right],\left[\begin{array}{llll}0 & 1 & \overline{1} & 0\end{array}\right]=\left(1 / \mathrm{S}_{11}\right)(\mathrm{GPa})$ & 91 & 71.5 & 98 \\
\hline$\left[\begin{array}{llll}0 & 0 & 0 & 1\end{array}\right]=\left(1 / \mathrm{S}_{33}\right)(\mathrm{GPa})$ & 111 & 135 & 125 \\
\hline \multicolumn{4}{|l|}{ Poisson's ratio } \\
\hline 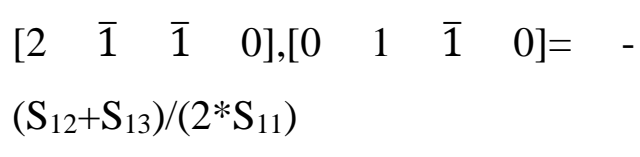 & 0.318 & 0.357 & 0.321 \\
\hline 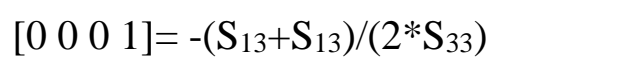 & 0.333 & 0.25 & 0.307 \\
\hline
\end{tabular}

The above parameters show that values calculated for $\left[\begin{array}{llll}0 & 0 & 0 & 1\end{array}\right]$ direction using EAM and MEAM potentials compare well with the experimental values. Molecular dynamics clearly predicts anisotropic behavior of mechanical properties of zirconium. The simulations are further used to calculate temperature dependence of lattice parameters and temperature dependent stresses.

\subsubsection{Variation of lattice parameters with temperature}

The lattice parameters calculated previously for zirconium structure is for zero velocities i.e. the energy of structure is only dependent on potential energy. But as we know, materials in the nuclear reactor core are exposed to very high temperatures, it is therefore important to test the potential at various temperatures and compare it with experimental values. The variation of lattice parameter with temperature is calculated and compared with experimental values in order to evaluate the behavior of potential when atomic velocities increase. First, the energy of the structure is fully minimized to obtain a stable configuration of atoms. The minimized structure is then subjected to initial velocity so that the required temperature is reached in the system. Under NPT (Constant pressure and constant Temperature) ensemble the system is equilibrated until the temperature fluctuations are minimum and the pressure of the system is fluctuating close to zero 
bar. The lattice parameters are evaluated when the volume of the system is constant. Lattice parameters are calculated as shown in table 5

Table 5: Lattice parameters changes with temperature calculated for EAM and MEAM potentials and compared with experimental data

\begin{tabular}{|l|l|l|l|l|l|l|}
\hline Temperature(K) & \multicolumn{2}{|c|}{ MEAM } & \multicolumn{2}{c|}{ EAM } & \multicolumn{2}{c|}{ Experimental $^{[17]}$} \\
& $\mathrm{a}(\AA)$ & $\mathrm{c}(\AA)$ & $\mathrm{a}(\AA)$ & $\mathrm{a}(\AA)$ & \multicolumn{1}{c|}{$\mathrm{c}(\AA)$} \\
\hline 300 & 3.2273 & 5.2106 & 3.2299 & 5.1756 & 3.2331 & 5.1490 \\
\hline 500 & 3.2343 & 5.2219 & 3.2317 & 5.1811 & 3.2364 & 5.1575 \\
\hline 600 & 3.2373 & 5.2272 & 3.2334 & 5.1841 & 3.2380 & 5.1624 \\
\hline 700 & 3.2401 & 5.2326 & 3.2355 & 5.1872 & 3.2397 & 5.1678 \\
\hline 800 & 3.2427 & 5.2379 & 3.2382 & 5.1906 & 3.2414 & 5.1737 \\
\hline 900 & 3.2448 & 5.2440 & 3.2413 & 5.1940 & 3.2431 & 5.1800 \\
\hline 1000 & 3.2471 & 5.2494 & 3.2448 & 5.1978 & 3.2447 & 5.1869 \\
\hline 1125 & 3.2497 & 5.2570 & 3.2499 & 5.2034 & 3.2468 & 5.1963 \\
\hline
\end{tabular}

Molecular dynamics simulations predict increase of lattice parameters with increase of temperature. As seen from above table that increase of temperature shows increase of lattice parameters of HCP crystal of zirconium predicted by both EAM and MEAM potentials. The potentials show good stability in the structure even at higher temperatures.

\subsubsection{Anisotropic thermal expansion in zirconium using EAM and MEAM}

Variation of lattice parameters with temperature is calculated and compared with the experimental data .The Thermal expansion coefficients of zirconium as calculated with EAM and MEAM is observed to be in good agreement with experimental data. These values are used to calculate the variation of thermal expansion along ' $a$ ' and ' $c$ ' directions of HCP crystal as shown in Figure 12 (a) and (b). 

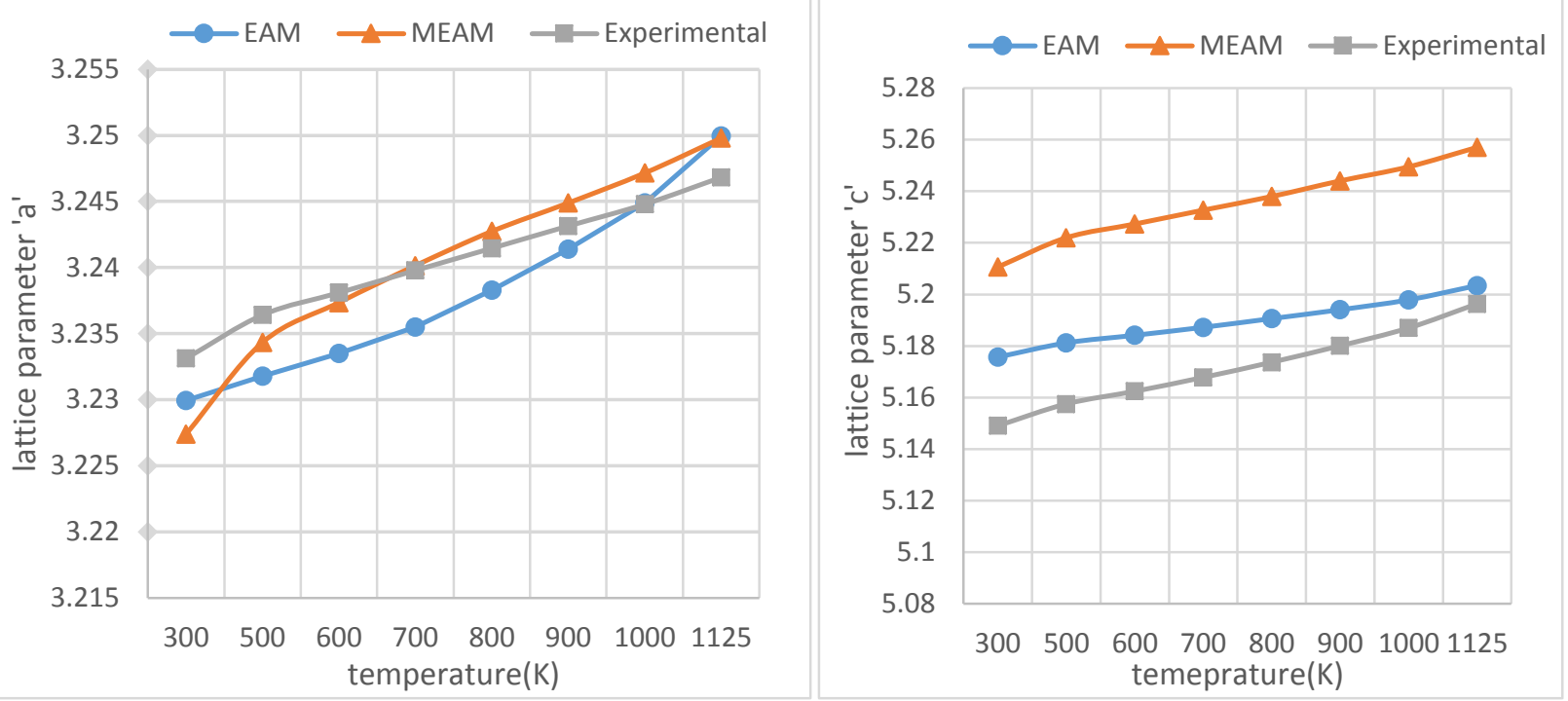

Figure 12: (a) Variation of lattice parameter 'a' with temperature (b) Variation of lattice parameter 'c' with temperature

These values are used to calculate variation of lattice parameter in ' $a$ ' and 'c' directions. From the minimized lattice parameters of zirconium, EAM potential $\left(\mathrm{a}_{\mathrm{T}} / \mathrm{a}_{\min }\right)=1.001268(1 / \mathrm{K})$ and $\mathrm{c}_{\mathrm{T}} / \mathrm{c}_{\min }=1.004187(1 / \mathrm{K})$ and for MEAM potential $\left(\mathrm{a}_{\mathrm{T}} / \mathrm{a}_{\min }\right)=1.007436(1 / \mathrm{K})$ and $\mathrm{c}_{\mathrm{T}} / \mathrm{c}_{\min }=$ $1.0083(1 / \mathrm{K})$. Experimental calculations by Goldak et al [17] indicate $\left(\mathrm{a}_{\mathrm{T}} / \mathrm{a}_{\mathrm{min}}\right)=1.0032(1 / \mathrm{K})$ and $\mathrm{cT} / \mathrm{c}_{\min }=1.0057(1 / \mathrm{K})$. From the above values anisotropy thermal expansion is higher along ' $\mathrm{c}$ ' direction when compared to ' $a$ ' direction of zirconium and is in good agreement with experimental data.

\subsection{Structure of zirconium hydride}

\subsubsection{EAM (Zr) and EAM $\left(\mathrm{H}_{2}\right)$ mixing potential}

An initial structure of face centered tetragonal (fct) zirconium hydride is created with lattice parameters $\mathrm{a}=4.981 \AA$ and $\mathrm{c}=4.451 \AA$ and with hydrogen at tetrahedral sites. The structure consists of 324 atoms with $108 \mathrm{Zr}$ and 216 hydrogen atoms with periodic conditions applied in all directions as shown in figure 13. The tests here are performed in order to find a stable lattice parameters and stable structure. 


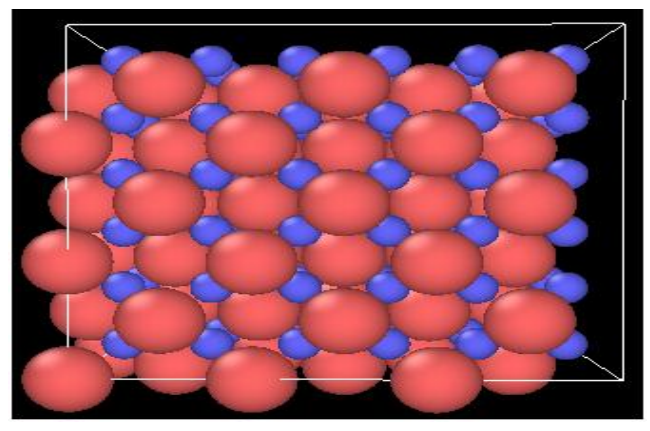

Figure 13: Structure of face centered hydride zirconium (red) and hydrogen (blue) in tetrahedral sites

\subsubsection{Failure of EAM $(\mathrm{Zr})$ and $\operatorname{EAM}\left(\mathrm{H}_{2}\right)$ mixing potential}

An initial setup zirconium hydride was made to calculate various parameters such as lattice parameter and stiffness values. A mixing potential is used for $\mathrm{Zr}$ with EAM potential and with hydrogen potential. A structure used in simulation is made with $108 \mathrm{Zr}$ atoms and 216 atoms of hydrogen. Upon minimizing this structure the hydrogen atoms are still unstable and finally the structure becomes unstable as shown in figure 14 and 15 respectively.

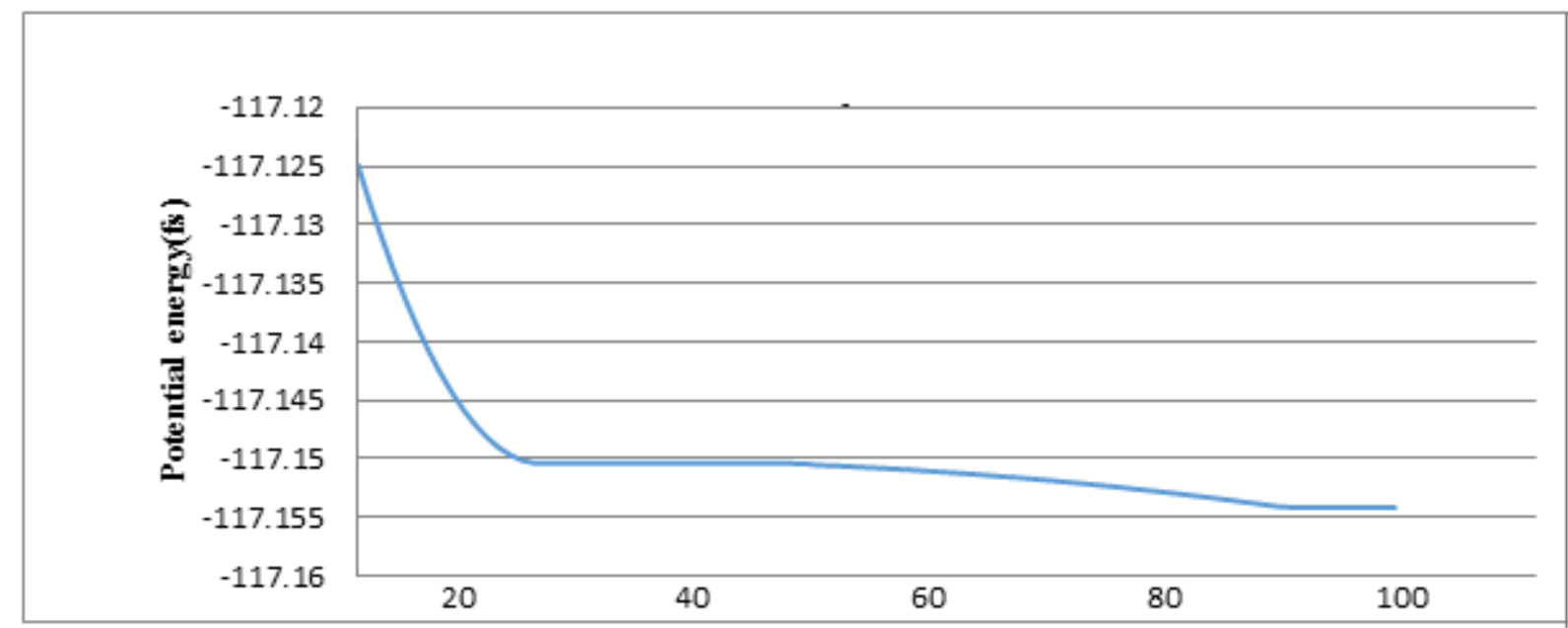

Timesteps

Figure 14: Minimized energy for hydride using EAM potential 


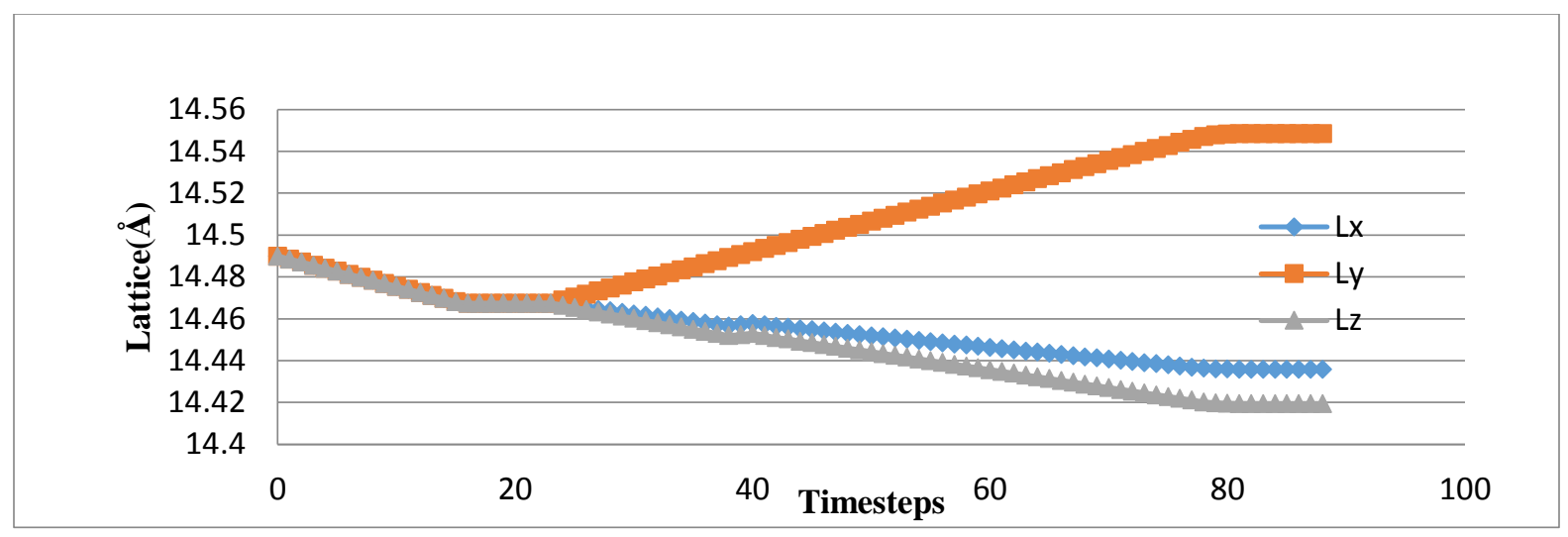

Figure 15: Minimized lattice parameters for hydride using EAM potential indicates unstable lattice structure. Lx, Ly and Lz represent lattice parameters of the structure

\section{Stiffness calculations using EAM $(\mathrm{Zr})$ and EAM $\left(\mathrm{H}_{2}\right)$ mixing potential}

The stiffness of EAM $(\mathrm{Zr})$ and EAM $\left(\mathrm{H}_{2}\right)$ is further tested in order check the stability of the potential using minimized structure. Hydrogen is placed in all tetrahedral sites of zirconium and structure is tested for calculation of stiffness values using script developed by Aidan Thompson. The calculated stiffness values $\mathrm{C}_{11}=121.07 \mathrm{GPa}, \mathrm{C}_{12}=126.79 \mathrm{GPa}, \mathrm{C}_{44}=-65.79 \mathrm{GPa}$. The Negative value along $\mathrm{C}_{44}$ direction indicates instability given by potential along that direction. So EAM potential in this research cannot be used for zirconium hydride.

\subsubsection{Test of stability of zirconium hydride using MEAM potential}

Face centered tetragonal (FCT)

FCT zirconium hydride is tested for convergence and lattice parameters. The MEAM potential shows good convergence and forms a stable FCC structure upon minimization as shown in figure 16 and 17 respectively.

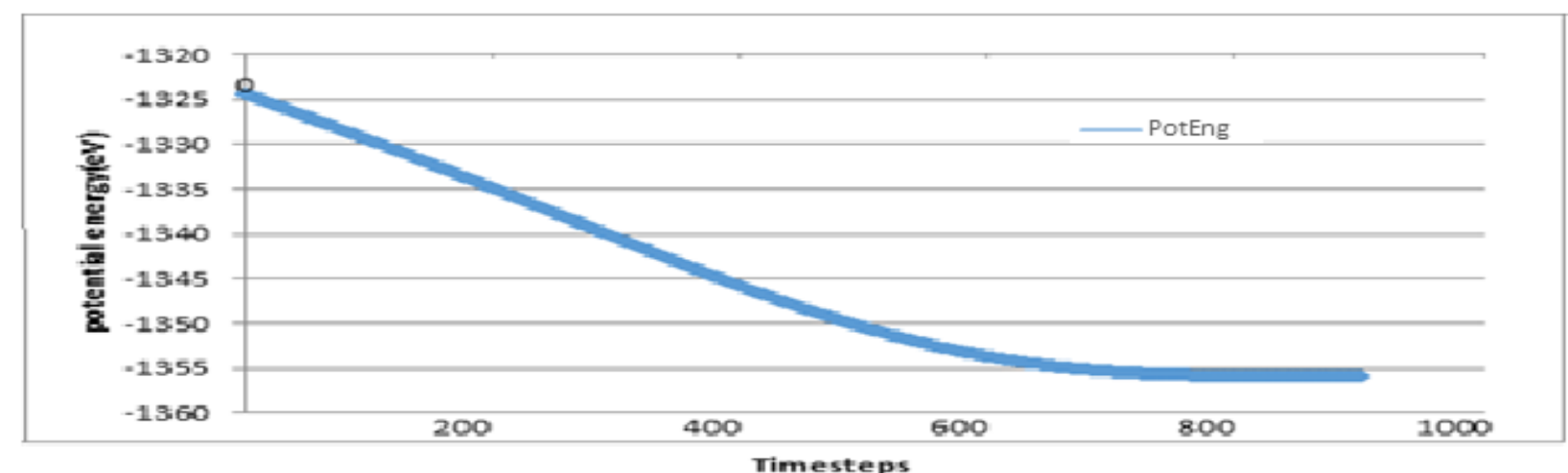

Figure 16: Convergence of potential energy for face centered hydride (fct) using MEAM potential 


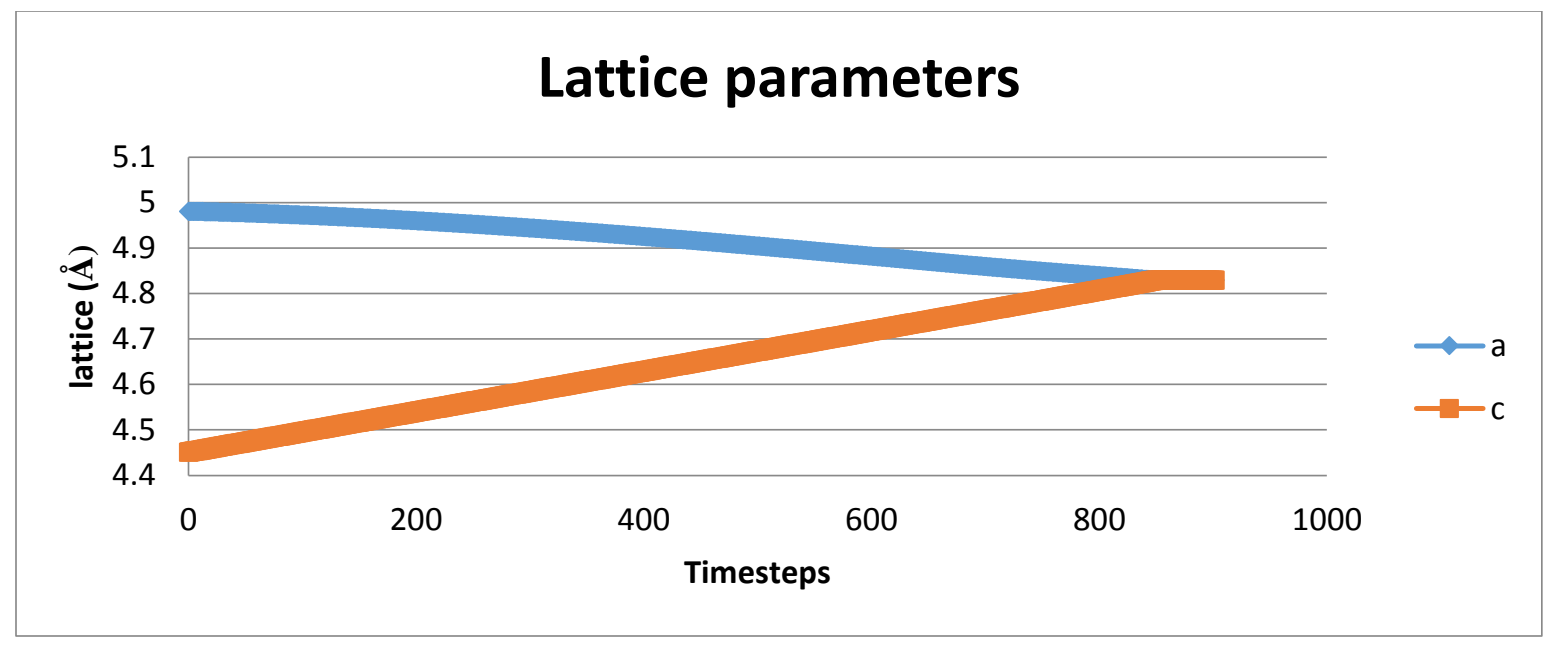

Figure 17: Convergence of lattice parameters from face centered tetragonal to face centered cubic hydride using MEAM potential along ' $a$ ' and 'c' directions

Face centered cubic (FCC) zirconium hydride

The convergence of energy and lattice parameters for FCC zirconium hydride is tested and is compared with FCT (figure 16 and 17) to FCC converted structure. They both converge to same potential energy and also same lattice parameters as shown in figure 18. This shows that the potential predicts FCC hydride as a stable structure. From the minimized energy structure the lattice parameters for hydride is calculated as shown in table 6.

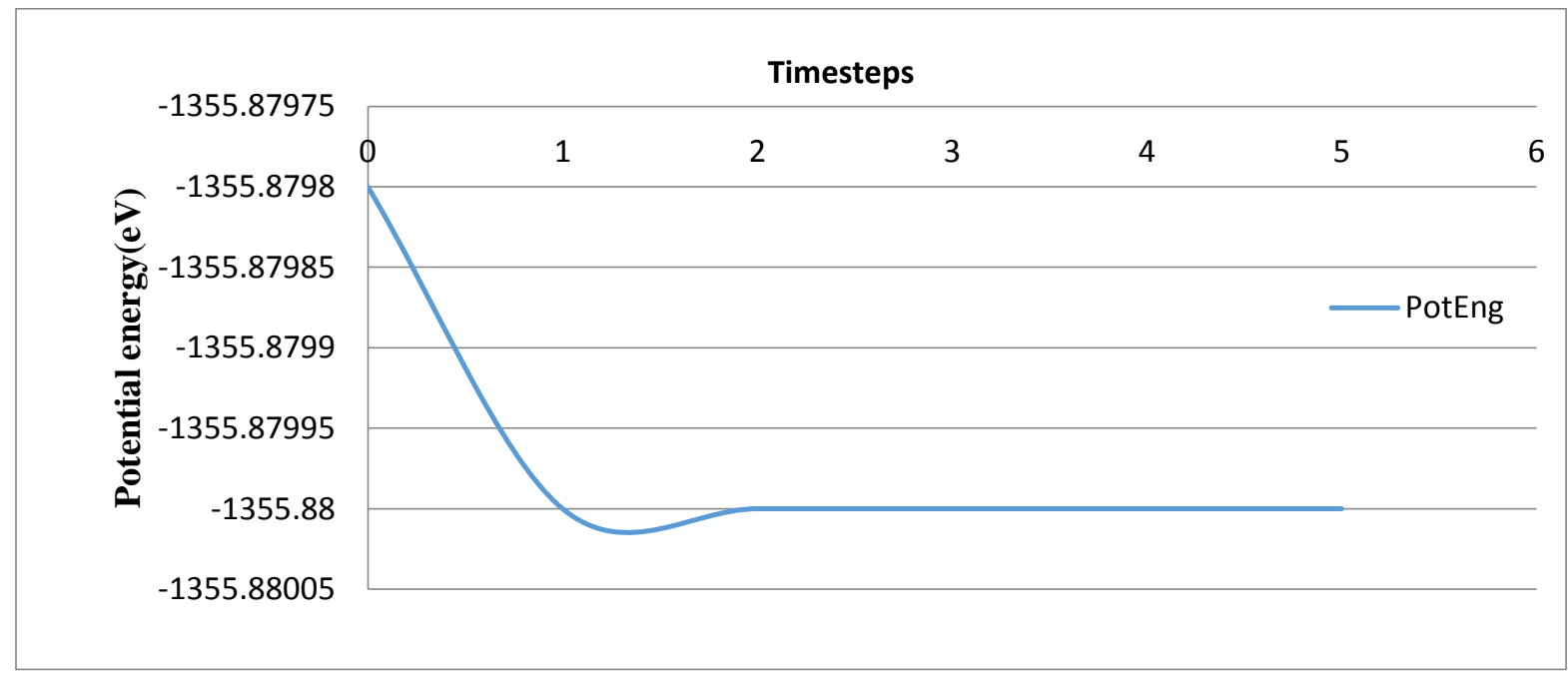

Figure 18: Convergence of the potential energy for face centered cubic (fcc) using MEAM 
Table 6: Comparison of lattice parameters $(\AA)$ and cohesive energy (eV/atom) with experimental values

\begin{tabular}{|l|l|l|l|l|}
\hline Compound & Lattice Parameter & $\begin{array}{l}\text { MEAM } \\
\text { Calculated }\end{array}$ & Experimental & $\begin{array}{l}\text { Theoretical } \\
\text { calculations }\end{array}$ \\
\hline$\delta-\mathrm{ZrH}_{2}$ & $\mathrm{a}(\AA)$ & 4.829 & $4.78^{[21]}$ & $\begin{array}{l}4.83^{[12,23]} \\
4.817^{[13]}\end{array}$ \\
\hline$\varepsilon-\mathrm{ZrH}_{2}$ & $\mathrm{a}(\AA)$ & - & $4.985^{[13]}$, & \\
& & & $4.975^{[20],}$ & $5.017^{[12]}$ \\
\hline & $\mathrm{c}(\AA)$ & - & $4.430^{[13]}$, & $4.447^{[12]}$ \\
& & & $4.447^{[20],}$ & \\
\hline
\end{tabular}

The above results indicate a good agreement of lattice parameters with experimental and theoretical data MEAM potential used in this research are correct for zirconium hydride and can be used for further analysis on mechanical properties of hydride.

\subsection{Stiffness values for $\delta-\mathrm{ZrH}_{2}$}

The minimized structure is used to check for stiffness constants for $\delta-\mathrm{ZrH}_{2}$ and compare obtained data with experimental values to verify the potential used. The structure is elongated in a particular direction until the elastic limit is reached and this is followed by energy minimization after each step of deformation, and then the same procedure is applied to the compression. Figure 19 shows directions of FCC hydride in which stiffness is calculated as shown in table 7.

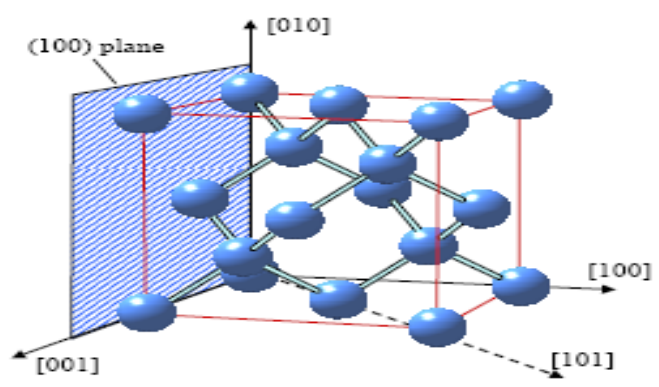

Figure 19: Face centered cubic structure hydride showing different crystal directions 
Table 7.Stiffness constants for $\delta-\mathrm{ZrH}_{2}$ structure and comparison with theoretical data

\begin{tabular}{|l|l|l|}
\hline $\begin{array}{l}\text { Stiffness Constants } \\
(\mathrm{GPa})\end{array}$ & $\begin{array}{l}\text { Calculated } \\
(\mathrm{GPa})\end{array}$ & Theoretical values $^{[13]}$ \\
\hline $\mathrm{C}_{11}(\mathrm{GPa})$ & 209.91 & 210 \\
\hline $\mathrm{C}_{12}(\mathrm{GPa})$ & 89.94 & 96 \\
\hline $\mathrm{C}_{44}(\mathrm{GPa})$ & 20.41 & 26 \\
\hline
\end{tabular}

The stiffness values show a good agreement with the theoretical values as there are no experimental data available. From the stiffness matrix we calculate mechanical properties and compare them with theoretical calculations as shown in table 8 and 9 .

Table 8: Young's Modulus and Poisson's ratio of zirconium hydride

\begin{tabular}{|l|l|l|}
\hline Direction[expression] & $\begin{array}{l}\text { Young's } \\
\text { Modulus } \\
(\mathrm{GPa})\end{array}$ & $\begin{array}{l}\text { Poisson's } \\
\text { Ratio }\end{array}$ \\
\hline $\mathrm{E}_{1}(100)\left[\left(\mathrm{C}_{11}-2^{*} \mathrm{C}_{12} * \mathrm{C}_{12}\right) /\left(\mathrm{C}_{11}+\mathrm{C}_{12}\right)\right]$ & 156 & 0.2999 \\
\hline $\begin{array}{l}\mathrm{E}_{2}(110) \\
{\left[4 *\left(\left(\mathrm{C}^{2}{ }_{11}+\mathrm{C}_{12} \mathrm{C}_{11}-2 \mathrm{C}^{2}{ }_{12}\right) \mathrm{C}_{44}\right) / 2 \mathrm{C}_{44} \mathrm{C}_{11}+\mathrm{C}^{2}{ }_{11}+\mathrm{C}_{12} \mathrm{C}_{11}-2 \mathrm{C}^{2}{ }_{12}\right]}\end{array}$ & 69 & - \\
\hline $\mathrm{E}_{3}(111)\left[3 * \mathrm{C}_{44}\left(\mathrm{C}_{11}+2 * \mathrm{C}_{12}\right) /\left(\mathrm{C}_{11}+2 \mathrm{C}_{12}+\mathrm{C}_{44}\right)\right]$ & 58.18 & - \\
\hline
\end{tabular}

Table 9: Calculated mechanical properties in comparison with theoretical calculations

\begin{tabular}{|l|l|l|}
\hline Mechanical properties & Calculated & $\begin{array}{l}\text { Theoretical } \\
\text { calculations }^{[13]}\end{array}$ \\
\hline Young's Modulus(GPa) $\left[\left(\mathrm{E}_{1}+\mathrm{E}_{2}+\mathrm{E}_{3}\right) / 3\right]$ & 94.39 & 93 \\
\hline Shear Modulus(GPa) $\left[\left(\mathrm{C}_{11}-\mathrm{C}_{12}\right) / 3\right]$ & 39.99 & 33 \\
\hline Bulk Modulus(GPa) $\left[\left(\mathrm{C}_{11}+2 * \mathrm{C}_{12}\right) / 3\right]$ & 130 & 130 \\
\hline
\end{tabular}

The above results indicate a good agreement of calculated mechanical properties with theoretical data from literature. The obtained results show that zirconium hydride has much higher stiffness 
and lower Young's modulus than pure zirconium which indicates that hydride is a brittle material.

\subsection{Variation of Lattice parameter with Temperature}

The Face centered cubic structure of zirconium hydride is then used to calculate the variation of lattice parameter with temperature to check for stability at higher temperatures. At $300 \mathrm{~K}$ the lattice parameters are $4.8578 \AA$ and at $800 \mathrm{~K}$ the lattice parameters are $4.909 \AA$. Increase of lattice parameters seen with increase in temperature and the structure remained stable. There are no experimental values to compare variation of lattice parameters with temperature.

\subsection{Conclusions}

- Minimized lattice parameters and cohesive energies for $\alpha-\mathrm{Zr}$ using MEAM and EAM potentials are found to be in good agreement with experimental data.

- The stiffness values, Young's Modulus, Poisson's ratio, bulk Modulus for $\alpha-\mathrm{Zr}$ is calculated using MEAM and EAM and compared with literature data, it was found to be in good agreement.

- Both EAM and MEAM potentials used in this research are validated for $\alpha-\mathrm{Zr}$ and further tested for presence of hydrides in order to evaluate the best potential for $\alpha-\mathrm{Zr}$ and the hydride.

- Thermal expansion of zirconium using EAM and MEAM potentials is found to be in good agreement with experimental data

- Stable face centered cubic structure using MEAM potential with lattice parameters 4.829 $\AA$ are obtained.

- Comparison of lattice parameters and stiffness constants for zirconium hydride with experimental data shows satisfactory agreement. Mechanical properties of hydride are calculated and hydride has higher bulk modulus compared to zirconium which means that hydride is brittle.

- MEAM potential used in this research for zirconium hydride is found to be very good and is further used in applications like stress analysis, diffusion and interfacial fracture and processes of hydride reorientation. 


\section{CHAPTER 5}

\section{STRESS ANALYSIS IN ZIRCONIUM USING MEAM AND EAM POTENTIALS}

During the reactor operating conditions zirconium is subjected to high pressure and temperatures. The variation of stresses with temperature is an important factor, because it determines the strength of zirconium and time to failure of the reactor components.

\subsection{Uniaxial tensile test for $\alpha-\mathrm{Zr}$ using MEAM}

We now investigate the variation of stresses under different temperatures using MEAM potential .Initially the structure and strain rates are tested in order to optimize the structure for variations in the temperature. The periodic boundary conditions are applied along $\mathrm{x}, \mathrm{y}$ and $\mathrm{z}$ directions. Initially, the structure is fully minimized and then it is equilibrated at the required temperature using NPT ensemble (constant pressure and temperature). A strain rate $0.005 \AA$ picoseconds is chosen with 6400 zirconium atoms. The equilibration is done at constant temperature and pressure. Then NPT (Constant atoms, constant pressure and temperature) is applied in $\mathrm{x}$ and $\mathrm{y}$ directions so as to calculate stresses in the $\mathrm{z}$ direction i.e. [0001] crystal direction. The variation of stress with temperature for zirconium is calculated using MEAM as shown in figure 20.

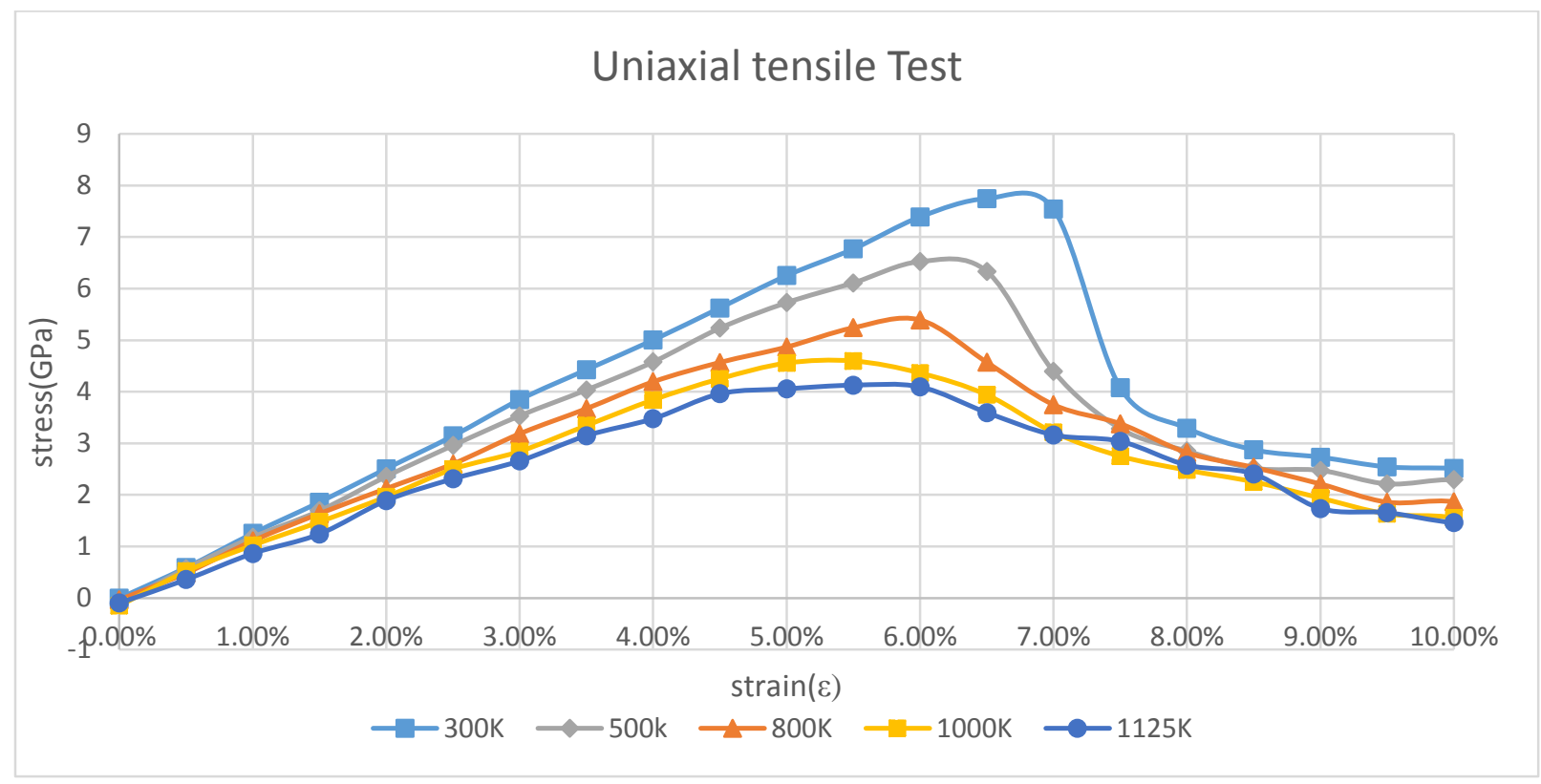

Figure 20: Variation uniaxial tensile stress for zirconium using MEAM potential at different temperatures 
As seen in the above graph the stresses are higher at lower temperatures and drop to lower values at higher temperatures as the material is plastically deformed. With the increase in temperature the yielding point is early. At 300K, molecular dynamics predicts yielding takes place at $7 \%$ strain and at $1125 \mathrm{~K}$ yielding is $4.5 \%$.

\subsection{Uniaxial compression test for $\alpha$-Zr using MEAM}

A compression test on zirconium using MEAM potential is conducted in order to see variation of stress with temperature and also to calculate stiffness constant along [0001] direction. A similar approach with compressive strain rate of $-0.005 \AA$ Apicoseconds is applied on the structure. The compression test are conducted at temperature range of (300-1125) K. Figure 21 shows changes of compressive stress with temperature.

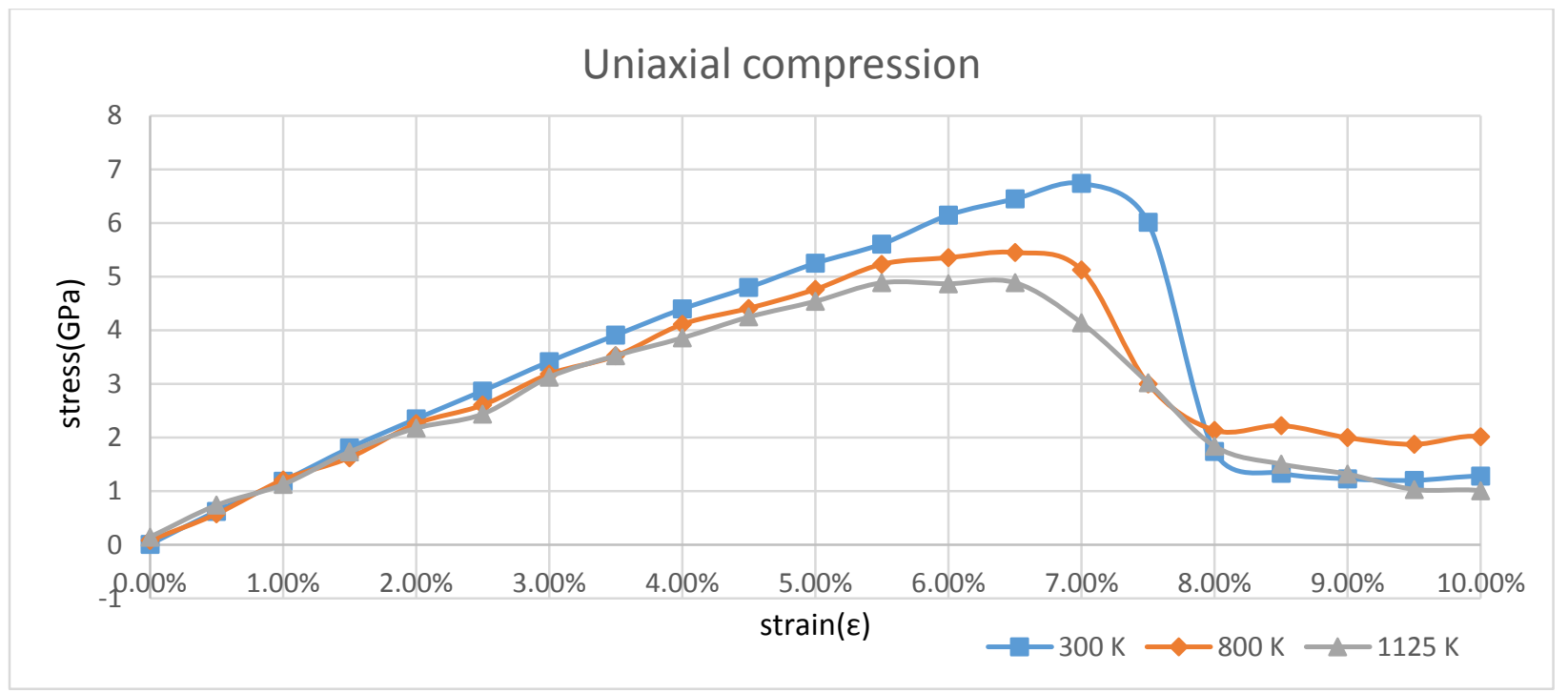

Figure 21: Variation uniaxial compressive stress for zirconium using MEAM potential at different temperatures

\subsection{Anisotropic stress of zirconium at $300 \mathrm{~K}$}

MEAM potential is used to evaluate the anisotropy of zirconium and see the variation of stress with direction, [11 $\overline{2}$ 0] and [0001] directions were chosen in order to compare stress and yielding point in the zirconium. Figure 22 show that [0001] has higher stress at the same strain rate and temperature. 


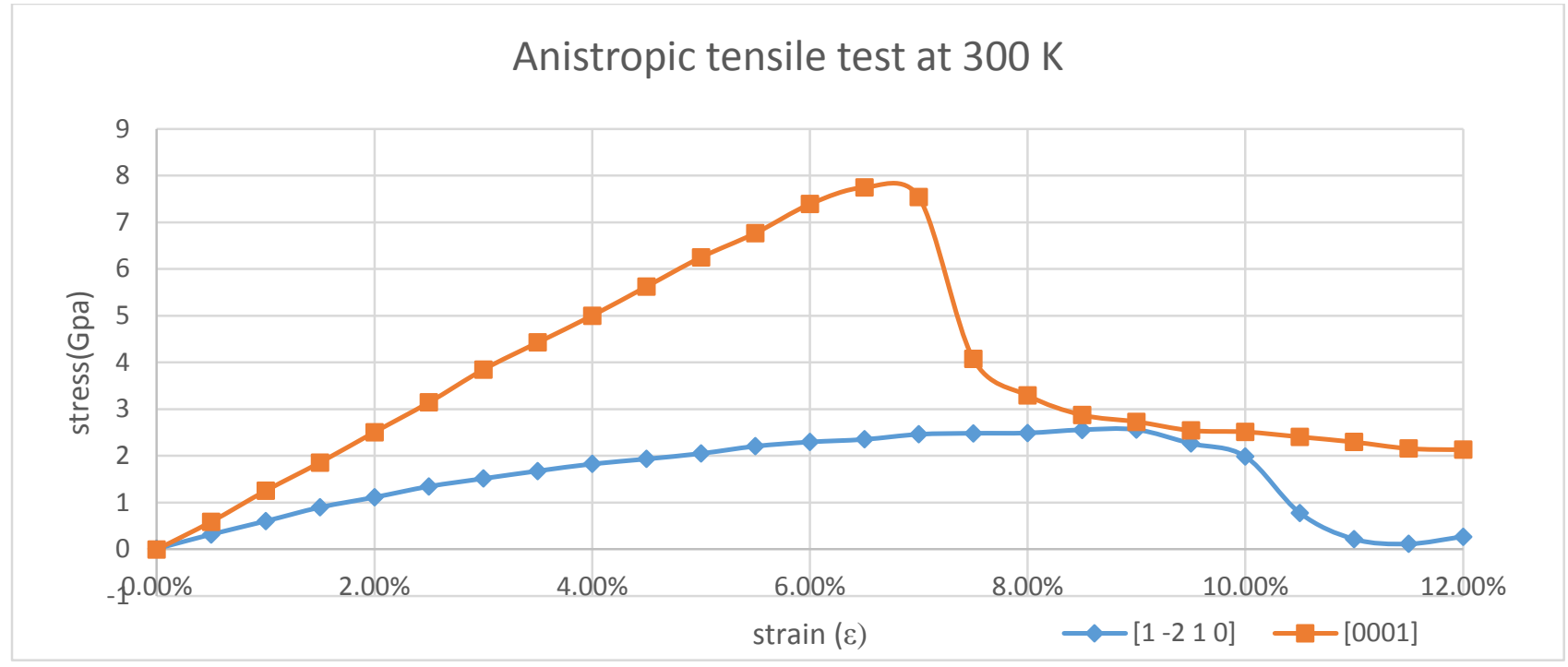

Figure 22: Anisotropic variation of stress for zirconium at constant temperature.

The above figure also indicates that failure of the material takes place earlier when tensile stress is applied along [1-2 10$]$ when compared to [0001].

\subsection{Uniaxial tensile test for $\alpha-\mathrm{Zr}$ using EAM}

Since EAM potential can be still used to test pure zirconium, we calculated stress strain curves for different temperatures. Similar calculations were performed using EAM potential. Tensile stress-strain curves calculated for different temperatures are plotted as shown in figure 23.

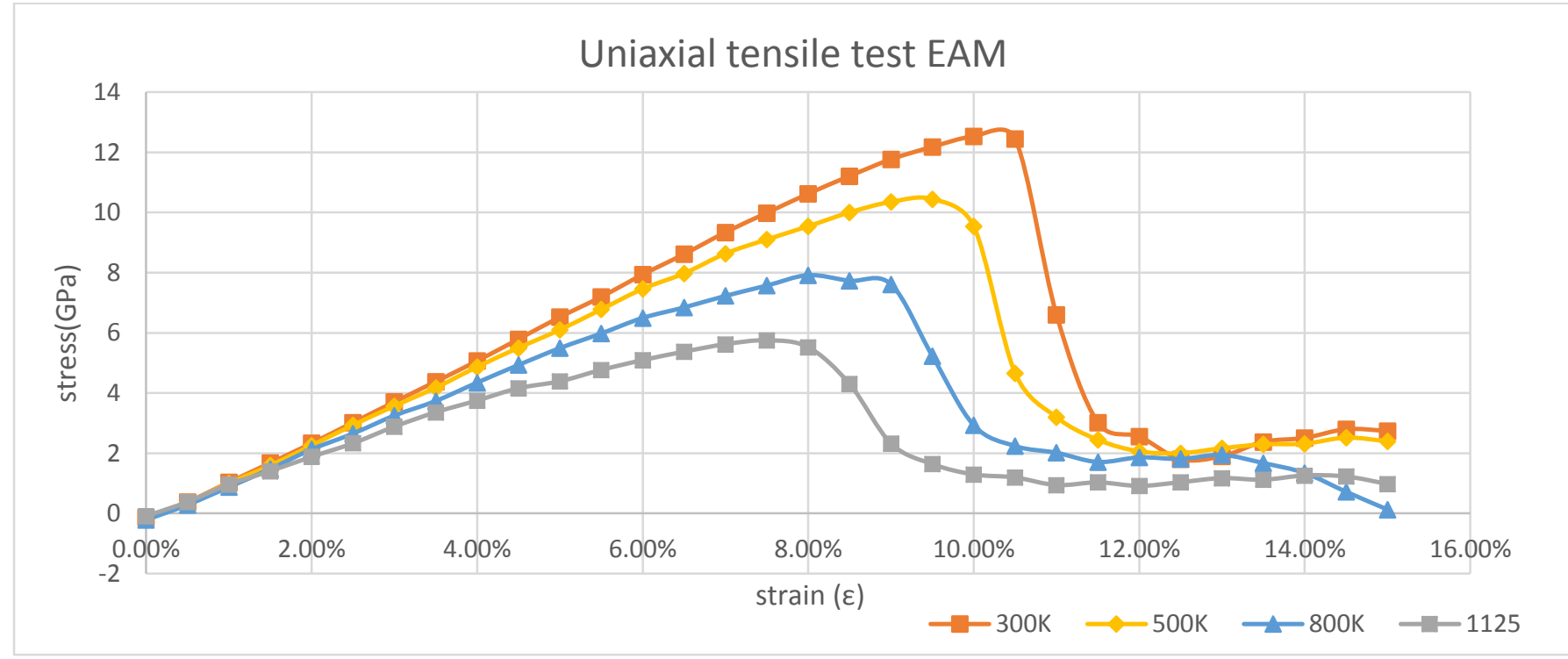

Figure 23: Uniaxial tensile stress-strain curves of zirconium calculated for different temperatures using EAM potential 
A similar variation of deformation with increase in temperature is observed when EAM potential is used. However, these MEAM potential curves fail earlier than those calculated using EAM potential. For example the tensile strength calculated with MEAM at $300 \mathrm{~K}$ is $8.0 \mathrm{GPa}$ while EAM is $12.44 \mathrm{GPa}$. The maximum deformation with MEAM potential at $300 \mathrm{~K}$ could reach $6.5 \%$ while with EAM elongation is $10.0 \%$

\subsection{Uniaxial compressive test using EAM potential}

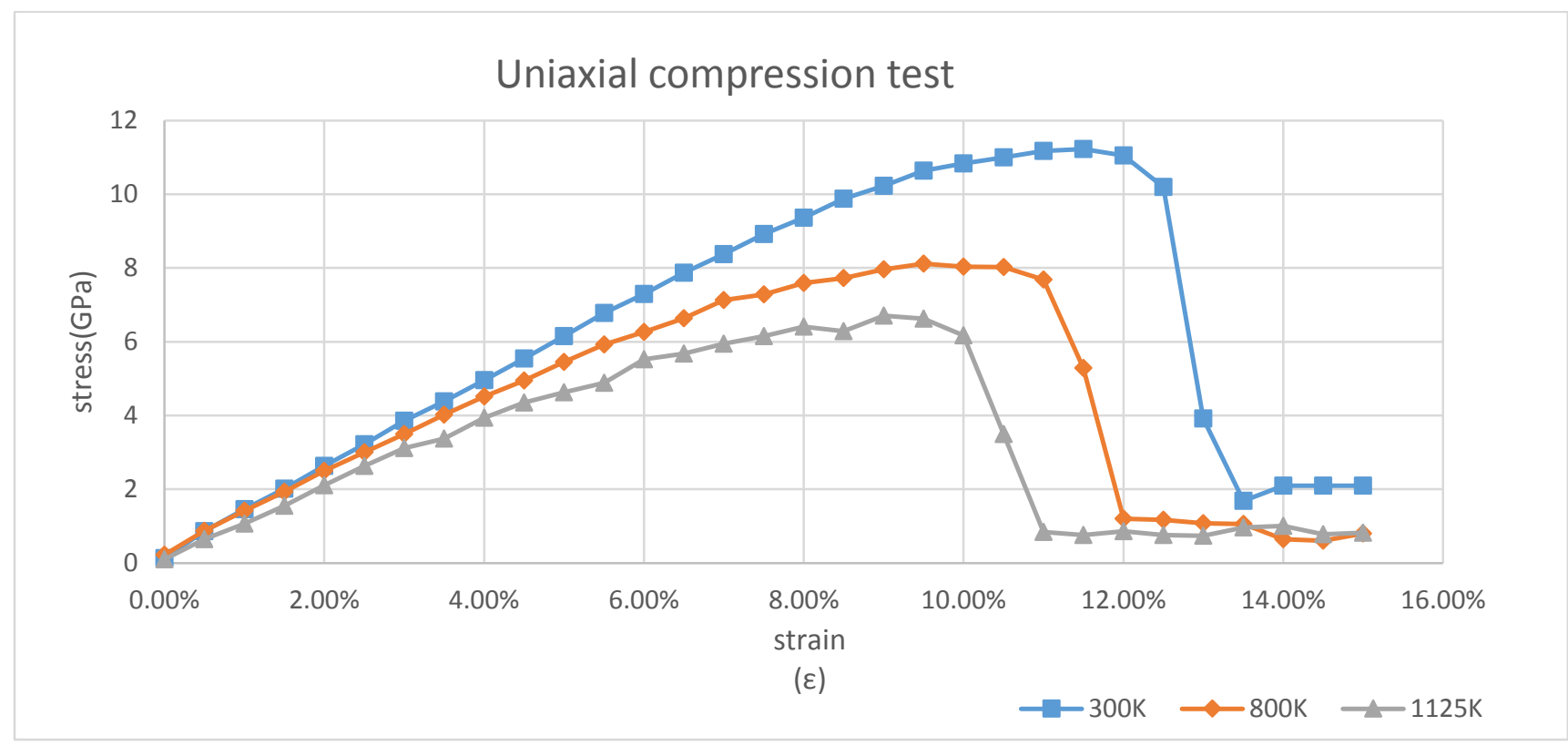

Figure 24: Uniaxial compression test on zirconium using EAM potential indicate early yielding with increase for a temperature range of $300-1125 \mathrm{~K}$

Compression tests performed on zirconium using EAM potential indicate yielding point decrease with increase in temperature as seen in figure 24. A similar trend has also been observed with MEAM potential. A maximum compressive stress of $11.22 \mathrm{GPa}$ at $300 \mathrm{~K}$ is observed using EAM potential on zirconium while using MEAM it was observed to be $6.73 \mathrm{GPa}$.Also the maximum strain before yielding starts using EAM is $12 \%$ while MEAM is $7 \%$

\subsection{Anisotropy of zirconium using EAM potential}

Differences in calculated mechanical properties are also observed when testing in different crystallographic directions as shown in figure 25. High stresses under the same strain are 
observed along [0001] direction and failure of material along both directions take place at around $10 \%$ strain.

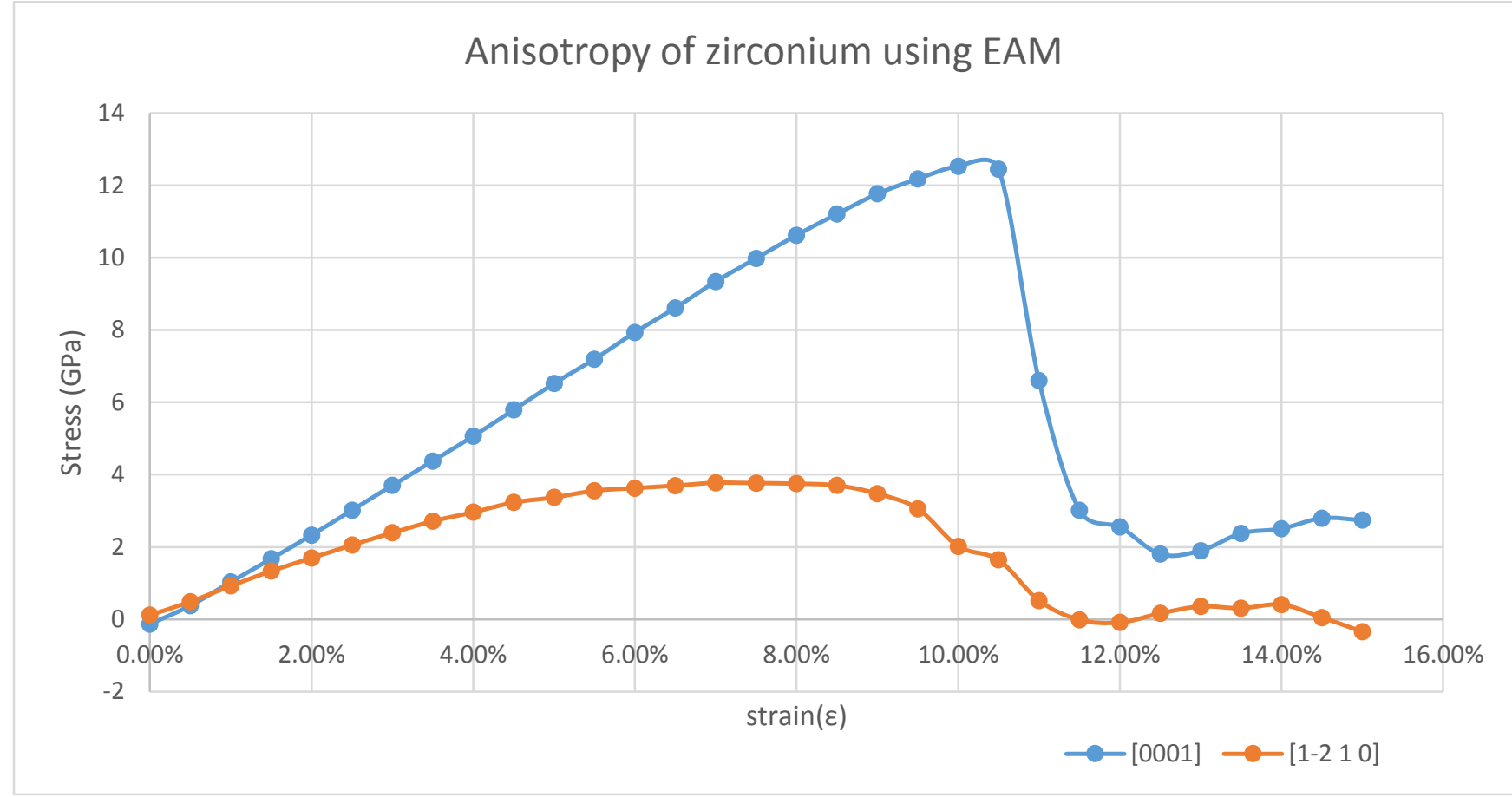

Figure 25: Stress-strain curves in different directions of zirconium calculated using EAM potential.

\subsection{Conclusions}

From the above analysis we can conclude that:

- Increase in temperature causes lower stress and early yielding.

- Tensile and compression stresses are lower at higher temperatures.

- Anisotropy of mechanical properties of zirconium is clearly observed using both EAM and MEAM potentials.

- Differences in Stress and Strain are observed between use of MEAM potential and EAM potential on zirconium. EAM potential seem to have higher stresses and is stable even at higher stains. 


\section{CHAPTER 6}

\section{DIFFUSION OF HYDROGEN IN ZIRCONIUM}

Hydrogen in zirconium or its alloys induces brittleness and limits fatigue life. The main source of hydrogen in zirconium alloys is the process of oxidation, also coolant and gases surrounding calandria tubes in nuclear reactors and hydrogen ingress during welding are sources of hydrogen. Diffusion coefficient of hydrogen was calculated at temperature ranges of $275^{\circ} \mathrm{C}$ to $700^{\circ} \mathrm{C}$ [49]. It has been demonstrated that diffusion coefficients is different along ' $a$ ' and 'c' direction of zirconium. Ab intio simulations were performed on zirconium oxide to study hydrogen motion into cracks and other defects [12]. In zirconium and its alloys brittleness is caused by hydrides solid transformation products formed in the interaction between the zirconium and by hydrogen. The understanding of hydrogen solubility and diffusion at atomistic level is not well understood and mechanism of reorientation of hydrides under stress is still not explained. However, because of imitation of experimental conditions, it is difficult to study the motion of hydrogen in zirconium. Molecular dynamics simulation is an important method for investigating behavior of hydrogen atoms in zirconium at atomic scale.

The diffusivity of hydrogen in metals is extremely high and is particularly high along interfaces and triple junction of grains, depends also on crystallographic direction. Diffusivity of hydrogen in zirconium increases with temperature by several orders of magnitude.

\subsection{Diffusion of hydrogen in zirconium using EAM and MEAM}

The diffusion of hydrogen in hexagonal structure of zirconium is modeled using 6400 atoms of zirconium and 100 atoms of hydrogen as shown in figure 26. MEAM, $\mathrm{Zr}-\mathrm{H}$ potential used were developed by Szpunar et al [13] and EAM zirconium (Zr) and EAM Hydrogen $(\mathrm{H})$ is used in this research. 

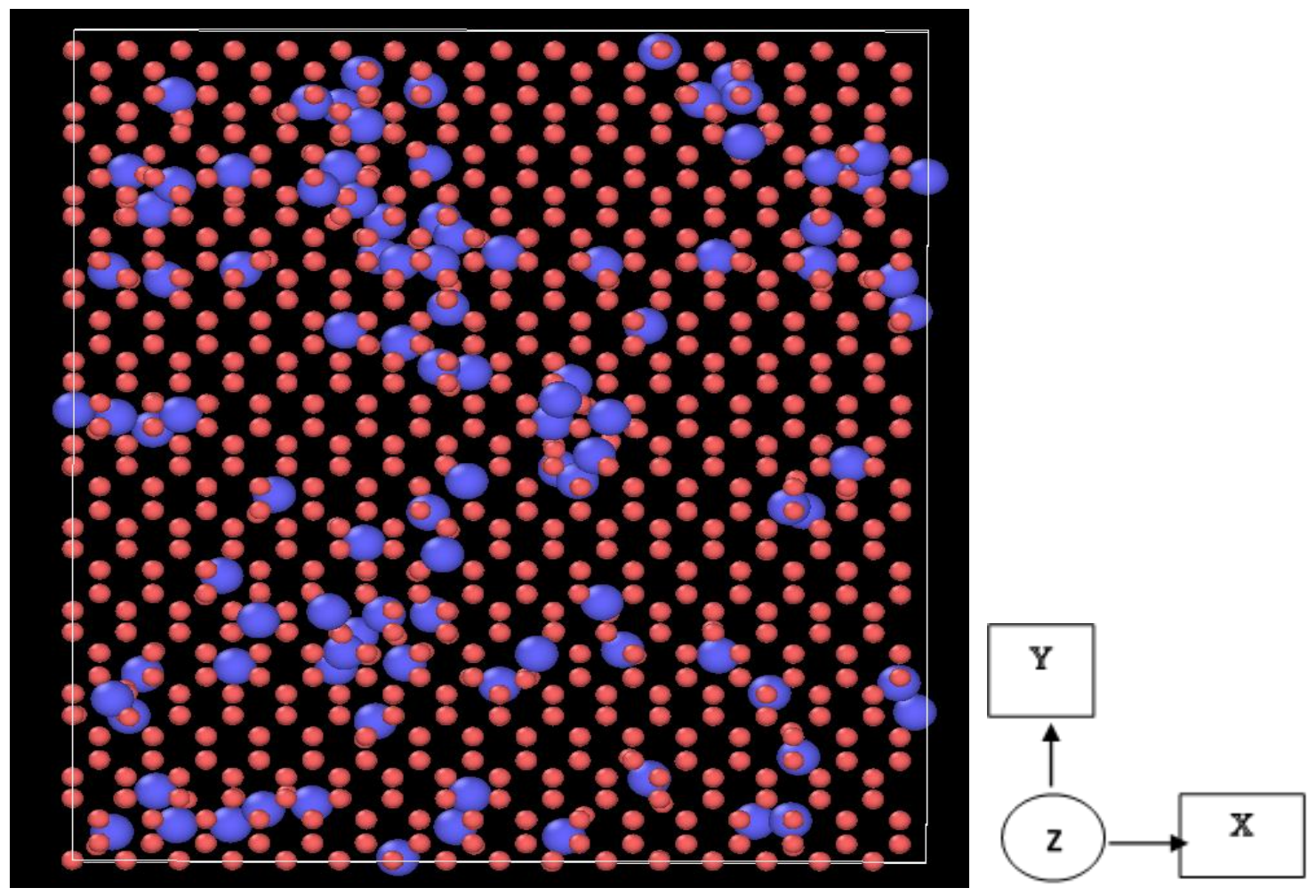

Figure 26 : Hydrogen (blue) is distributed randomly in zirconium (brown)

\subsection{Mean square displacement (MSD)}

Mean square displacement is the measure of the average distance a given atoms in a system is displaced. LAMMPS calculates MSD for group of atoms, including all complicated events where atoms are passing through limits of periodic boundaries used in simulation. Computation MSD in LAMMPS calculates a vector of four quantities. The first 3 elements of the vector are the squared $\mathrm{dx}, \mathrm{dy}, \mathrm{dz}$ displacements, summed and averaged over atoms in the group. The $4^{\text {th }}$ component is the total square displacement, i.e. $(d x * d x+d y * d y+d z * d z)$, summed and averaged over atoms in the group coefficients.

\subsection{Hydrogen displacement in zirconium}

A hexagonal closed packed zirconium is created with $6400 \alpha-\mathrm{Zr}$ atoms and 100 hydrogen atoms that are randomly distributed. Upon minimizing most of the hydrogen atoms prefers to occupy tetrahedral sites. The system is equilibrated at the desired temperature. Constant volume and energy (NVE) ensemble is applied in order to study hydrogen diffusion. Any drift of hydrogen atom was subtracted during mean square displacement calculation. The averaging of MSD is 
done for every 1000 timesteps up to $500 \mathrm{ps}$ timesteps for hydrogen atoms. The resultant average MSD as a function of time is shown in figure 27. This shows that diffusivity of hydrogen depends strongly on temperature and is higher at higher temperatures.

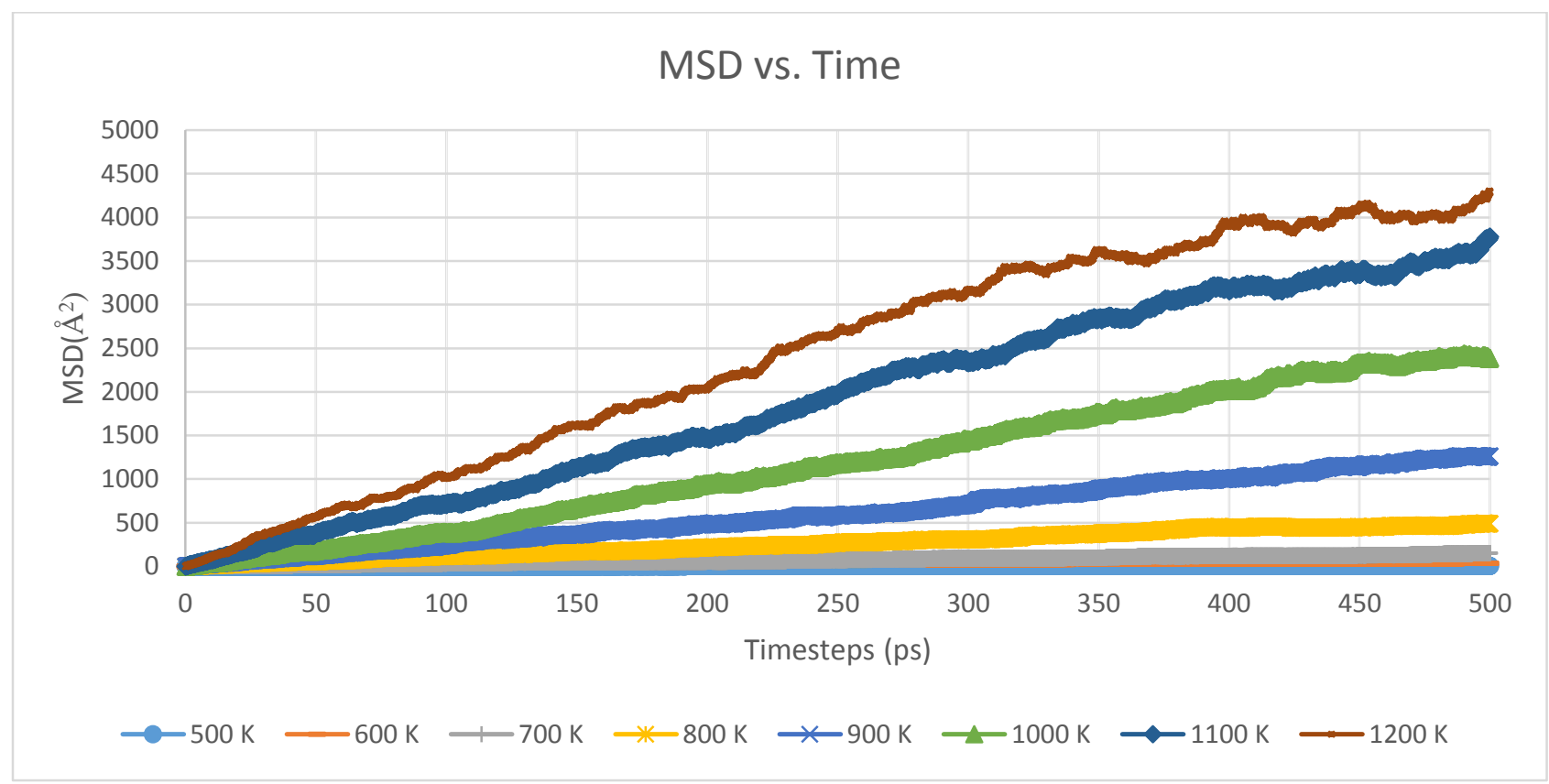

Figure 27: Variation of mean square displacement (MSD) of hydrogen inside zirconium for a temperature range of (500 -1200) K. Graph indicates hydrogen jumps (variation in slope) inside zirconium

MEAM is tested for hydrogen diffusion inside pure zirconium is tested for lower timesteps from 0.0001 to $0.00001 \mathrm{ps}$ in order to obtain a stable structure. As shown in figure 28(a) the structure seen to be unstable as zirconium atoms starts moving. However, EAM as figure 28(b) seen to be perfectly stable even at higher temperatures. Calculations for hydrogen diffusion in zirconium is done using EAM potential. 


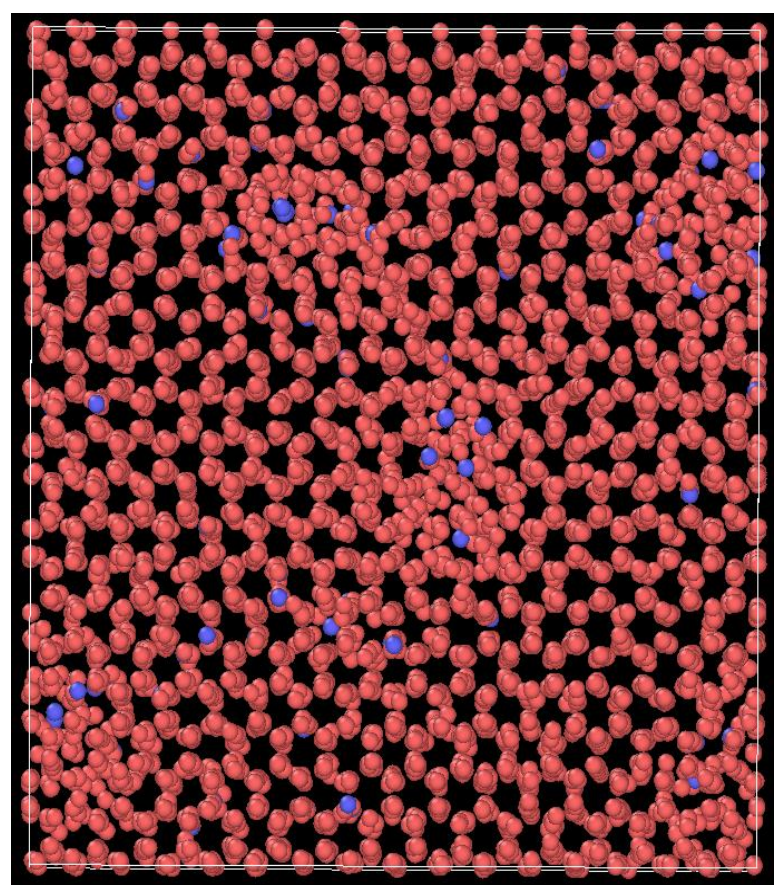

(a)

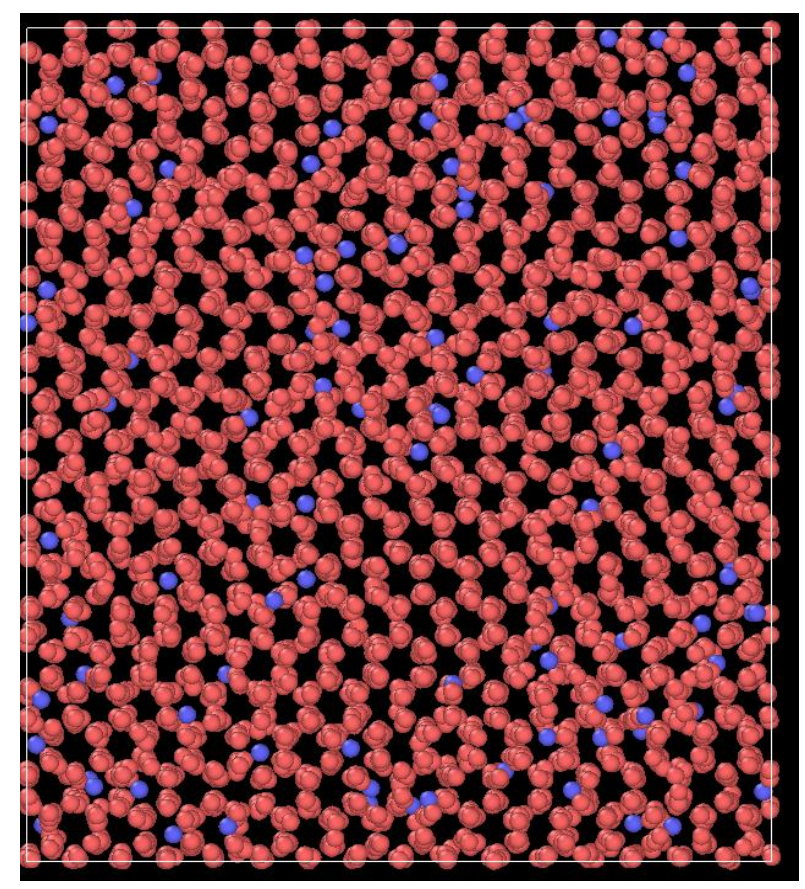

(b)

Figure 28: At $1200 \mathrm{~K}$ unstable structure using MEAM (b) EAM shows perfectly stable structure and higher displacements of hydrogen.

\subsection{Diffusion coefficients of hydrogen and activation energy of hydrogen}

The diffusion coefficients (D) are calculated using Einstein relation [6.3] as shown in equation 6.3

$$
D=\lim _{t \rightarrow \infty}\left\langle\frac{r^{2}(t)}{2 d t}\right\rangle
$$

Where $\left\langle r^{2}(t)\right\rangle=\left[r_{i}\left(t+t_{0}\right)-r_{i}\left(t_{0}\right)\right]^{2}$, $t_{0}$ is the average over all possible initial times, $d$ is the dimensionality of space ( $\mathrm{d}=3$ for bulk diffusion). The slope of plots of the MSD versus time has been measured for a temperature range of $(500-1200) \mathrm{K}$. The diffusion coefficient is calculated and compared with experimental data as shown in figure 29. 
Diffusion coefficients of hydrogen in $\alpha-\mathrm{Zr}$

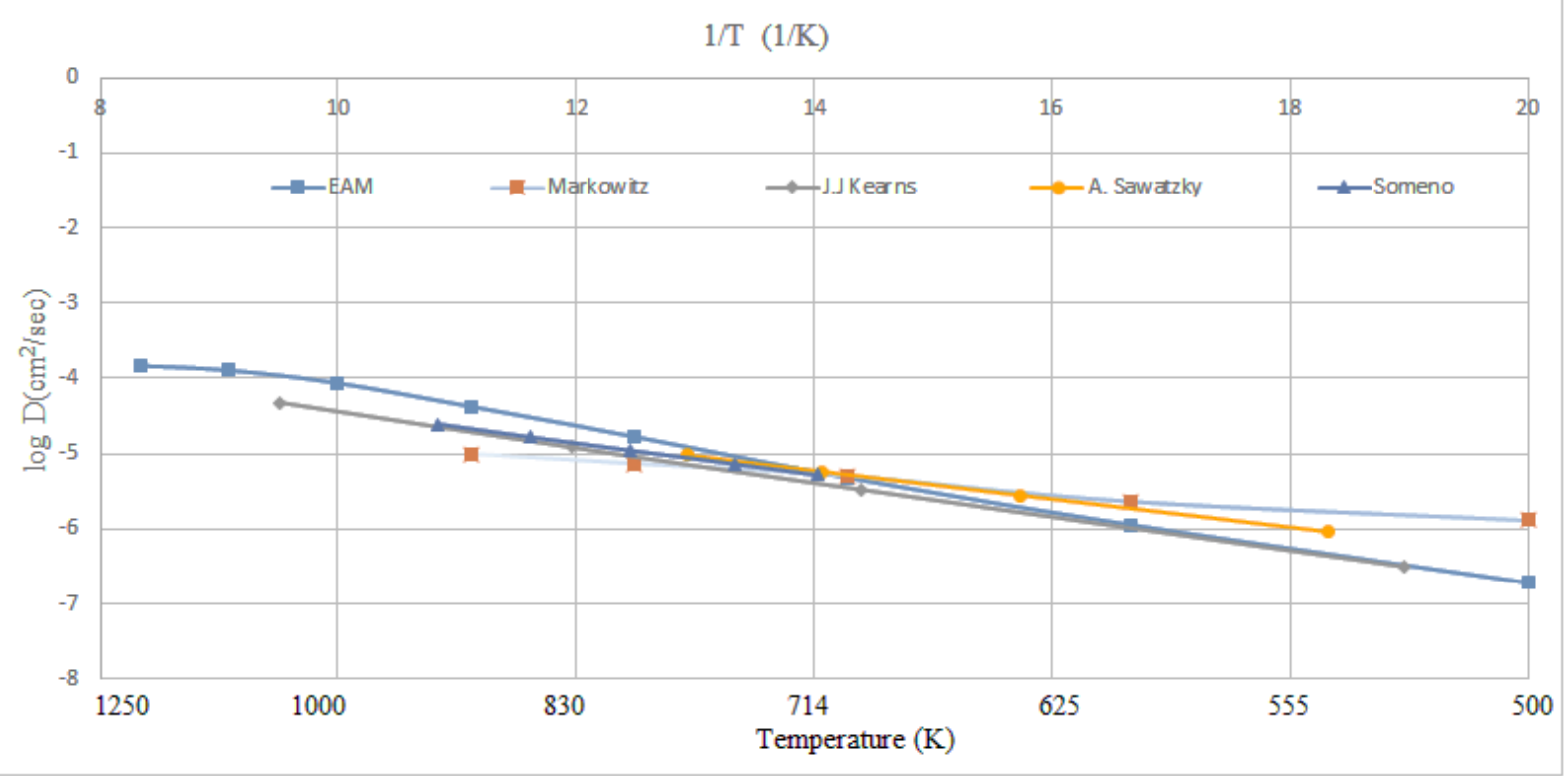

Figure 29: Arrhenius plot of hydrogen in zirconium for a temperature range of $(500-1200) \mathrm{K}$ indicates good diffusion coefficients of hydrogen when compared with the experimental data.

Activation energy of hydrogen is calculated for a temperature range 500- $1200 \mathrm{~K}$. As shown in table 10 calculated activation energy of hydrogen is in good agreement with available experimental data.

Table 10 : Calculated activation energy of hydrogen in zirconium is within the range of observed experimental data by various authors

\begin{tabular}{|l|l|}
\hline & Activation energy(kcal/ mol) \\
\hline EAM calculated & 11.302 \\
\hline Markowitz[61] & 1.6 to 6.5 \\
\hline Johnston[62] & 6.4 \\
\hline J.J Kearns[49] & 10.83 \\
\hline A. Sawatzky[60] & 3.8 to 6.1 \\
\hline Barbara et al(hydrogen in Nickel) & 11.618 \\
\hline
\end{tabular}




\subsection{Anisotropic Hydrogen diffusion coefficient}

Experimental results indicate hydrogen diffusion in zirconium single crystal is anisotropic [49]. The MSD of randomly distributed hydrogen atoms is calculated also in [11 $\overline{2} 0],\left[\begin{array}{llll}0 & 1 & \overline{1} & 0\end{array}\right]$ and [0001] crystal directions and the diffusion coefficient is calculated for these directions at various temperatures.

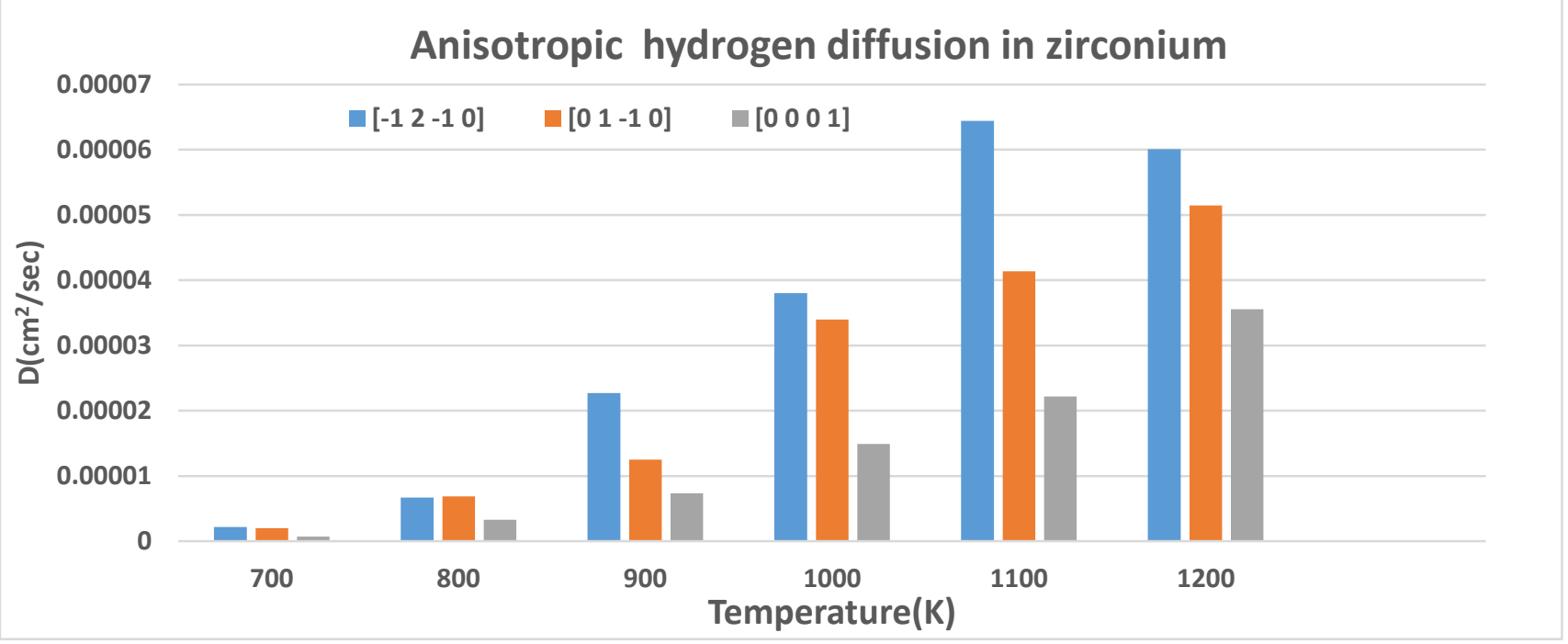

Figure 30: Anisotropic hydrogen diffusion coefficient's inside zirconium with increase in temperature

As shown in figure 30 hydrogen diffusion coefficients are higher along [1120], [lllll

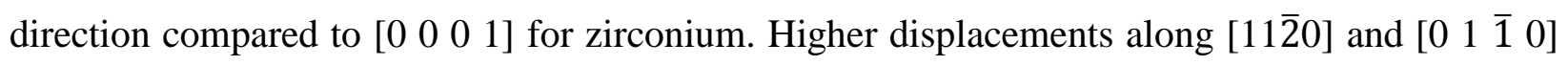
can lead to formation of hydrides along basal plane as observed experimentally [8].

\subsection{Strain induced diffusion of hydrogen in zirconium}

Many researchers have studied the effect of hydrides reorientation on the mechanical properties of the hydrides. But, the mechanism behind the hydrogen diffusion on stress at the level of molecular dynamics simulation of diffusion is still unclear. In the present study, the molecular model of hydrogen atoms in $\alpha$-zirconium is generated and this system is subjected to strain along [11 $\overline{2} 0]$ and [0001] directions of HCP directions of zirconium. The study of process of hydrogen diffusion in molecular dynamics can be studied through the process of strain dependent diffusion. 


\subsection{Hydrogen atoms in $\alpha$-zirconium}

In order to study the strain diffusion process hydrogen atoms are placed in the spherical hole inside the zirconium matrix. This spherical hole has radius of $1 \AA$ is made in zirconium. Figure 31 shows hole with hydrogen atoms inside $\alpha$-zirconium. The structure is then minimized until the pressure and energy of the system is minimum. Further the structure is equilibrated to $800 \mathrm{~K}$.
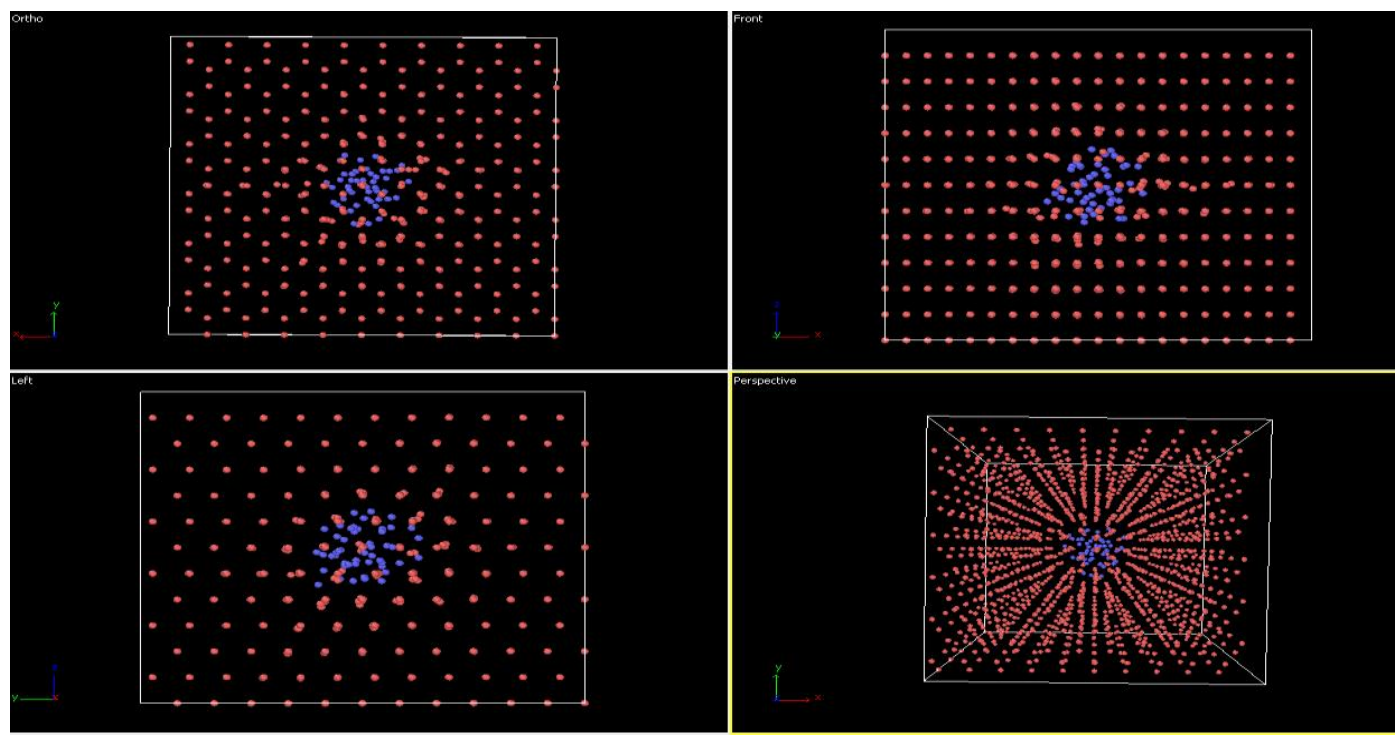

Figure 31: Different orthogonal views of hydrogen atoms (blue) placed in spherical box inside zirconium (brown) in order to study hydrogen diffusion with strain

\subsection{Strain along $[11 \overline{2} 0]$ direction of the structure}

The process of diffusion on application of strain is an important factor that helps to understand reorientation process in molecular dynamics simulation. The zirconium which contains hydride phase is strained along [11 $\overline{2} 0]$ and a temperature of $800 \mathrm{~K}$ is maintained in structure.

\subsubsection{Diffusion of hydrogen atoms before strain}

Initial displacements of hydrogen atoms are calculated after equilibration. The hydrogen atoms are seen to move in all the three directions as seen in figure 32. 


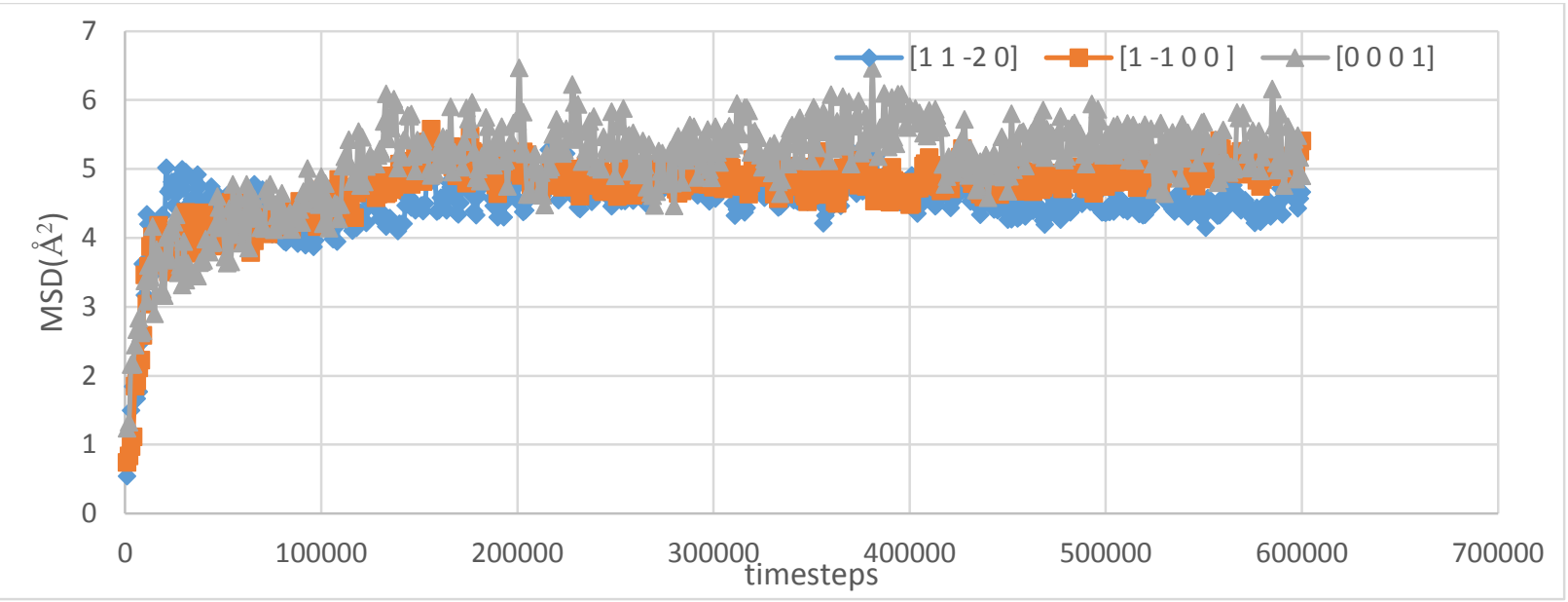

Figure 32: Hydrogen displacement is similar in all directions during equilibration and when no strain is applied

\section{$\underline{\text { 6.7.2 Calculation of Diffusion under uniaxial strain along [11 } 20] \text { direction }}$}

The structure is deformed uniaxial until the stress of $300 \mathrm{MPa}$ is reached. A $0.04 \%$ change in volume is observed on the application strain. The structure which is deformed under uniaxial tensile strain is then kept under NVT ensemble to calculate the mean square displacement (MSD) of hydrogen atoms. The mean square displacement of hydrogen atoms is calculated for timesteps of 5000000 ps after the structure is subjected to strain.

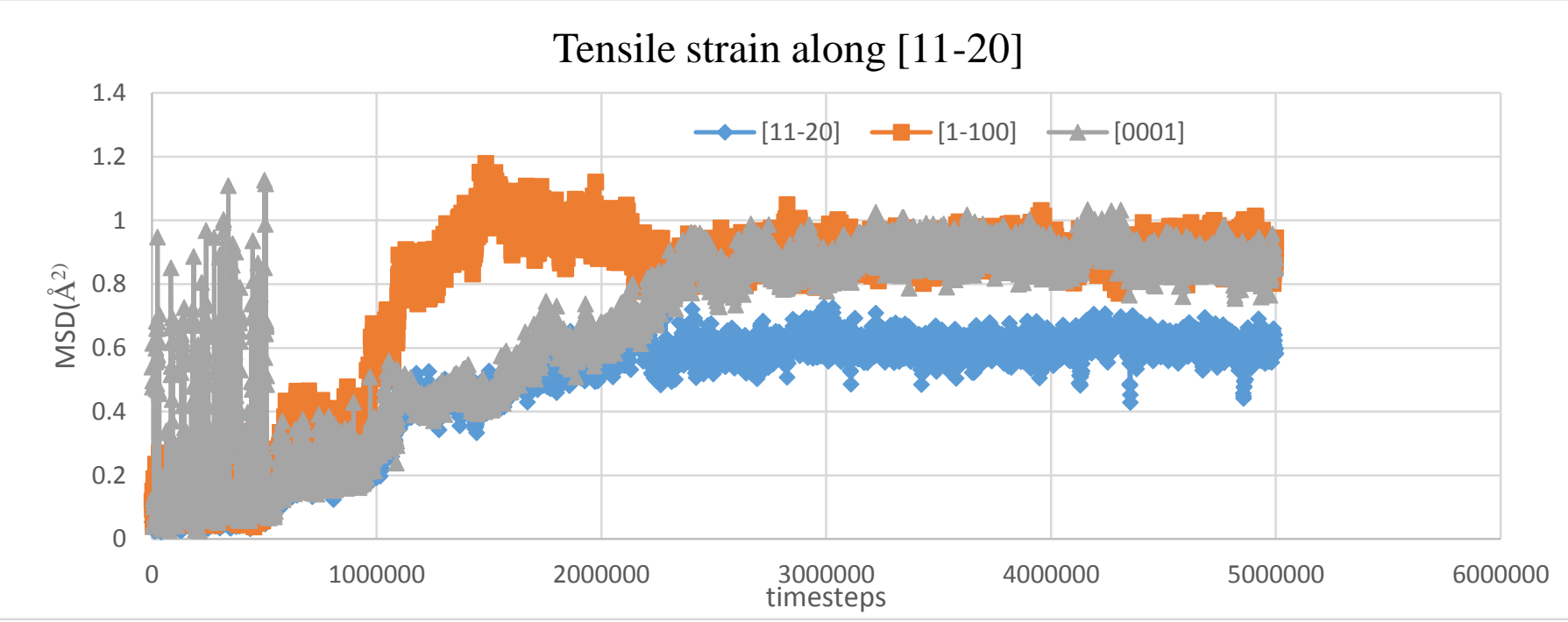

Figure 33: Mean square displacement of hydrogen atoms in 3 different directions of zirconium when applying the tensile strain along [1120].Jump in MSD indicate movement of hydrogen atoms. 
The above figure 33 shows the MSD displacement along three crystallographic directions on application of uniaxial stress along [1120], illustrates the hydrogen's preferentially moves along [0001] and [ $\left[\begin{array}{lll}1 & 1 & 0\end{array}\right]$ direction. The higher jump in MSD along [ $\left[\begin{array}{llll}1 & 1 & 0 & 0\end{array}\right]$ could be due to higher displacements of hydrogen along that direction. This observation can be used to explain the preferential formation of hydrides in that direction on application of tensile stress.

\subsubsection{Diffusion calculation under compression along [11 $\overline{2} 0]$ direction}

The structure is compressed along [11 20$]$ in order to observe the diffusion direction of hydrogen atoms. Uniaxial compression is applied along [112̄0] until the compression stress of $288 \mathrm{MPa}$ is reached. The strain of $0.25 \%$ is applied along [11 20 ] direction and then the deformed structure is used to calculate diffusion of hydrogen atoms. The figure 34 shows calculated MSD of hydrogen atoms diffusing along three crystallographic directions.

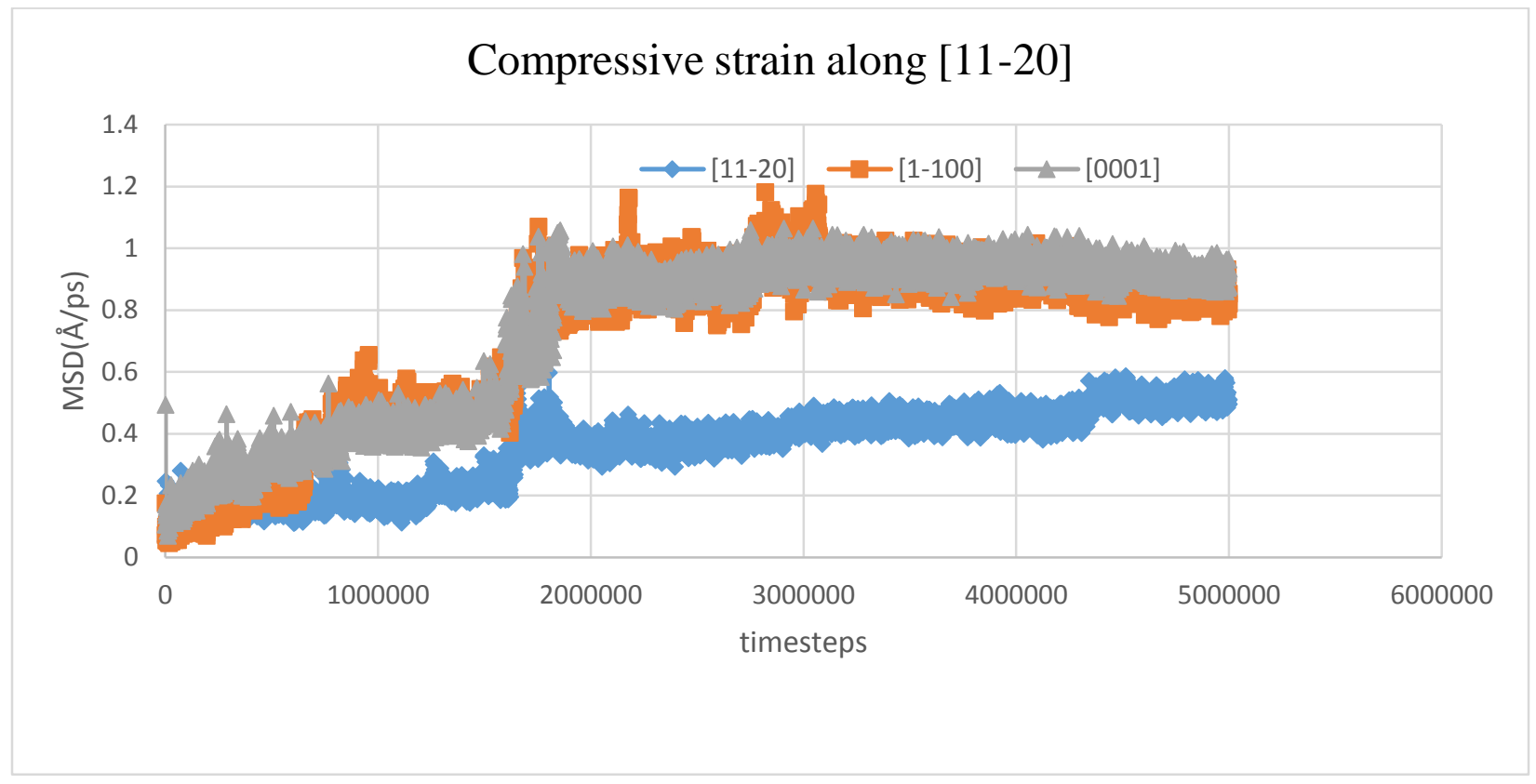

Figure 34: Mean square displacement of hydrogen atoms in 3 different directions of zirconium

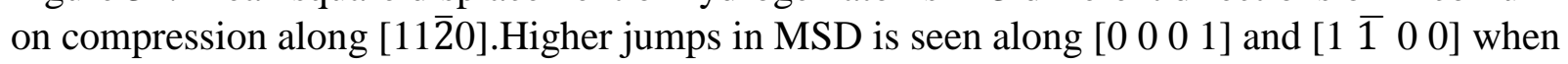
structure is compressed.

The compression along [11 $\overline{2} 0]$ direction shows hydrogen atoms moving in [0 $\left.\begin{array}{llll}0 & 0 & 0 & 1\end{array}\right]$ and $\left[\begin{array}{llll}1 & \overline{1} & 0 & 0\end{array}\right]$ crystal direction of zirconium similar to tensile stain. The jumps in MSD is due to movement of hydrogen along that direction. Variation of hydrogen diffusion due to compression and tensile stress calculated using Einstein equation is shown in table 11 
Table 11: Comparison of diffusion coefficient's on tension and compression strains.

\begin{tabular}{|l|l|}
\hline Type of strain & Diffusion Coefficient $(\AA / \mathrm{ps})$ \\
\hline Tensile & $4.01 \mathrm{E}-07$ \\
\hline Compression & $4.03 \mathrm{E}-07$ \\
\hline
\end{tabular}

The above table shows diffusion coefficient is slightly higher when compressive strain is applied than tensile.

\subsection{Strain along [0001] direction of the structure}

The structure is strained along [0001] direction in order to understand the effect of stress on diffusion along [0001] direction of crystal. A Uniaxial tensile strain is applied along [0001] until the strain of 0.004 is reached and the pressure inside the system is $612.06 \mathrm{MPa}$. The volume change of $0.14 \%$ is observed. The deformed system is further used to calculate mean square displacement of hydrogen atoms.

6.8.1. Calculation of diffusion under uniaxial tensile strain along [0001] direction The deformed structure is kept at constant volume using NVT ensemble and mean square displacement is calculated. A similar approach is used as in case of the uniaxial tension along [11̄̄0].

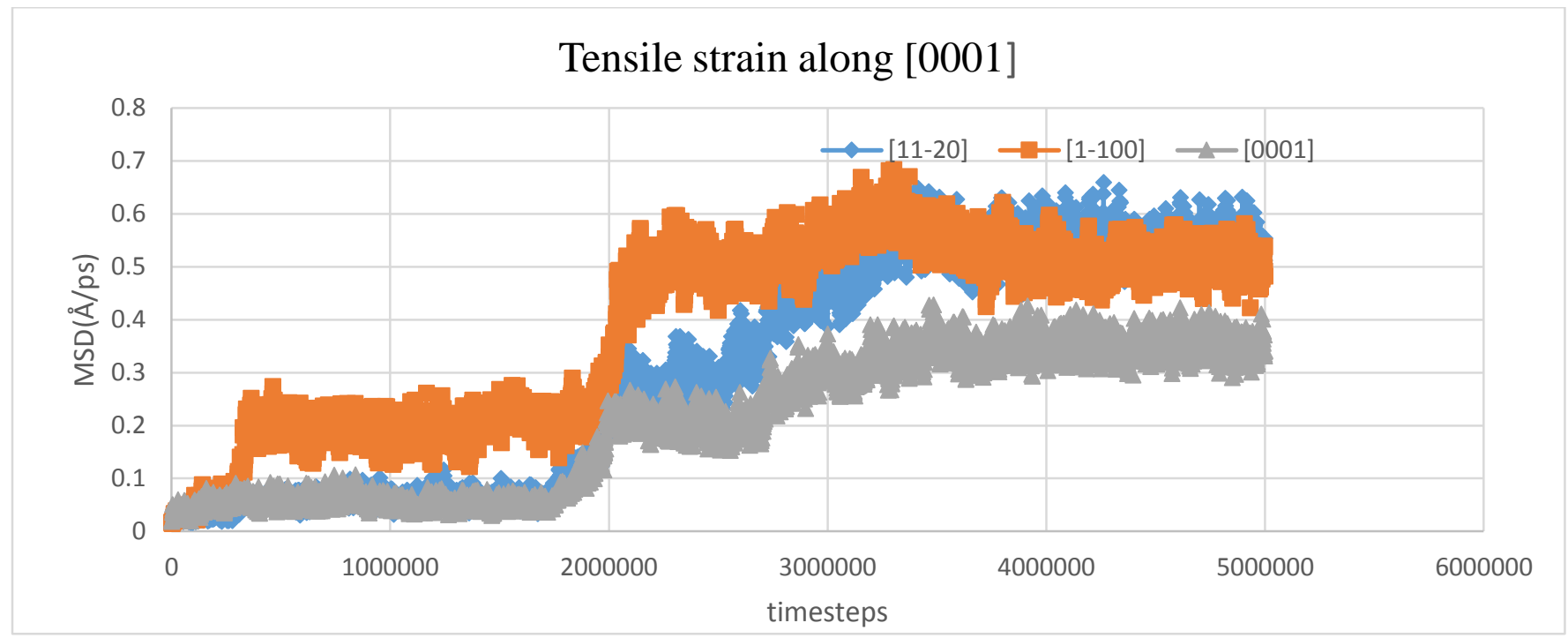

Figure 35: Mean square displacement of hydrogen atoms for tensile strain applied along [0001] direction in zirconium. Jumps are seen to be higher along [11 $\overline{2} 0]$ and [1 $\overline{1} 00]$ due to movement of hydrogen in that direction. 
The figure 35 shows that hydrogen jumps. Applying tension along [0001] direction the diffusion coefficient of hydrogen atoms is higher along [1120] and [1-100] directions. The hydrogen atoms prefer to move along these both directions.

6.8.2 Hydrogen diffusion calculation under compression stress along [0001] direction

The structure is compressed along [0001] in order to observe differences in the MSD of hydrogen atoms along different directions. The $0.25 \%$ strain is applied in compression along [0001] direction and then in this deformed structure the diffusion of hydrogen atoms is calculated, as shown in figure 36 .

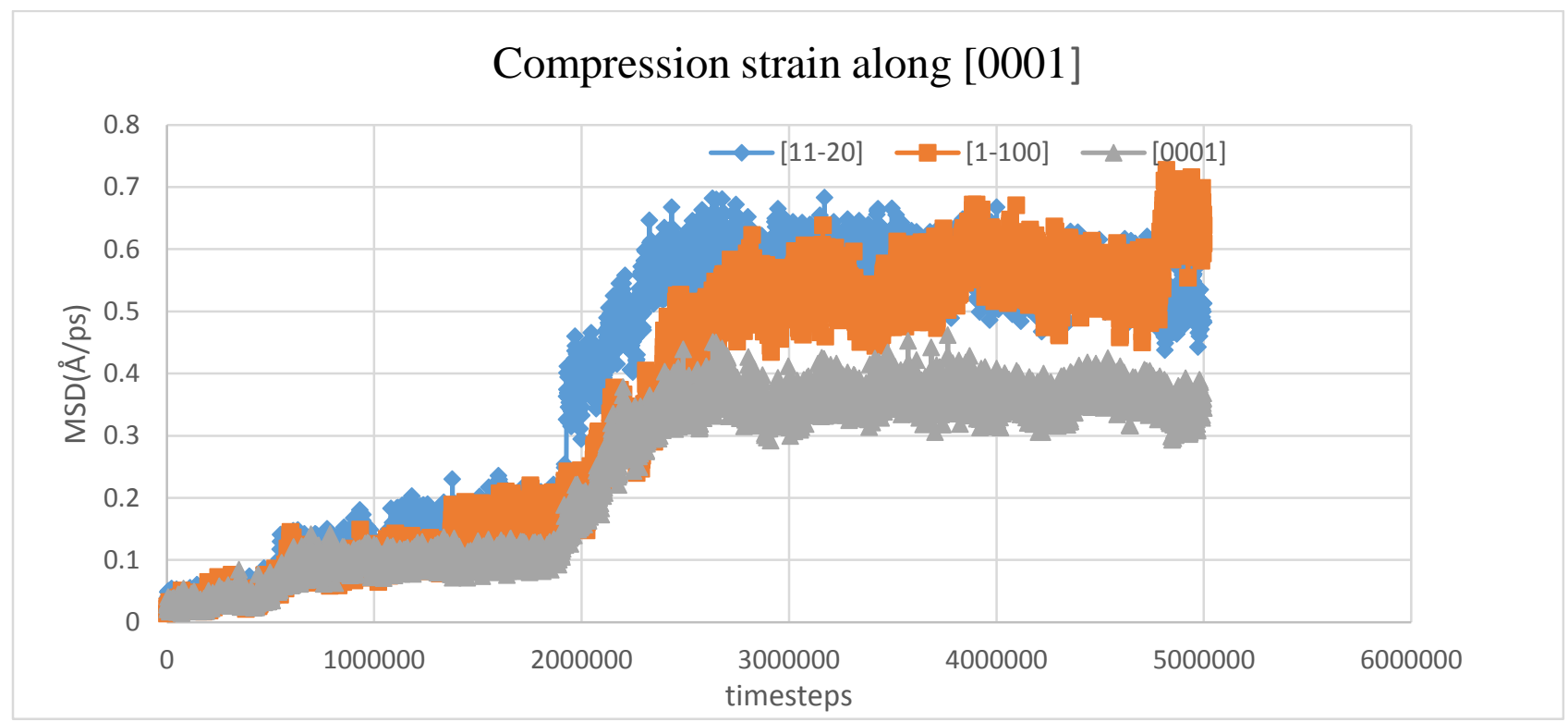

Figure 36: Mean square displacement of hydrogen atoms in 3 directions when compressed along [0001] direction of zirconium. Higher jumps in MSD indicate movement of hydrogen atoms along [ $[11 \overline{2} 0]$ and $\left[\begin{array}{llll}1 & \overline{1} & 0 & 0\end{array}\right]$.

The above graph indicates upon compression of structure the hydrogen atoms preferentially move in $[11 \overline{2} 0]$ and $\left[\begin{array}{ll}1 \overline{1} & 0\end{array}\right]$ direction. The table 12 shows that compression has higher effect of hydrogen diffusion along [0001] of zirconium.

Table 12 Diffusion coefficient of tension and compression

\begin{tabular}{|l|l|}
\hline Type of Strain & Diffusion coefficient $(\AA / \mathrm{ps})$ \\
\hline Tensile & $3.30 \mathrm{E}-07$ \\
\hline Compression & $3.57 \mathrm{E}-07$ \\
\hline
\end{tabular}




\subsection{Conclusions}

- Mean square displacement was used to calculate coefficients of hydrogen diffusion in zirconium. According to MD calculation diffusion of hydrogen increases with increase in temperature from $500 \mathrm{~K}\left(4.08 \mathrm{E}-08 \mathrm{~cm}^{2} / \mathrm{s}\right)$ to $1125 \mathrm{~K}\left(6.66 \mathrm{E}-07 \mathrm{~cm}^{2} / \mathrm{s}\right)$.

- Calculated activation energy is seen to in reasonable agreement with experimental data.

- Hydrogen diffusion coefficients are different along crystallographic directions. Higher diffusion coefficients of hydrogen were obtained along [11 $\overline{2} 0]$ and $\left[\begin{array}{llll}0 & 1 & \overline{1} & 0\end{array}\right]$ crystal direction.

- Molecular dynamics shows that hydrogen diffusion is affected by the compression and tensile strain applied to the $\alpha-\mathrm{Zr}$ single crystal system.

- Diffusion is higher under compressive strain than tensile strain.

- Since the calculation of hydrogen diffusion were performed on the system that simulate the orientation of crystals in $\alpha-\mathrm{Zr}$ based fuel cladding and pressure tubes in the fuel channel of nuclear reactors the results presented can be used to justify the reorientation of hydrides under stress. Such reorientation along radial direction contributes to embrittlement and failure of these tubes. 


\section{CHAPTER 7}

\section{STUDY OF ORIENTATION OF HYDRIDE}

Stress induced reorientation process of hydride has been studied extensively by many researchers [4, 27-30, 52, 53]. Measurements through EBSD [52] and Synchrotron [28] indicate that stress plays a major role in reorientation of hydride at the matrix of zirconium and zirconium hydride. The reoriented hydride is always seen to precipitate perpendicular to applied stress. Many possible explanations have been provided for the cause of this kind of behavior by hydrides. [9, 27- 30]. One theory suggests that diffusion of hydrogen inside hydride upon stress causes reorientation [28]. Other theory suggests that strains resulting from the volume misfit between hydrides and matrix are thought to be partly responsible hydride reorientation [53]. However, no clear evidence has been provided on the effect of strain on the oriented hydride itself. The research here provides an atomistic explanation on the cause of hydride reorientation through change in strain energy.

\subsection{Electronic structure of hydride}

In order to study the reorientation process the hydride is oriented as $\mathrm{x}\left[\begin{array}{lll}1 & \overline{1} & 0\end{array}\right], \mathrm{y}\left[\begin{array}{lll}1 & 1 & \overline{2}\end{array}\right]$ and oriented $z$ [ $\left.\begin{array}{lll}1 & 1 & 1\end{array}\right]$. The 3 -dimentsional view of hydride is shown in figure 37 total of 384 atoms are created.

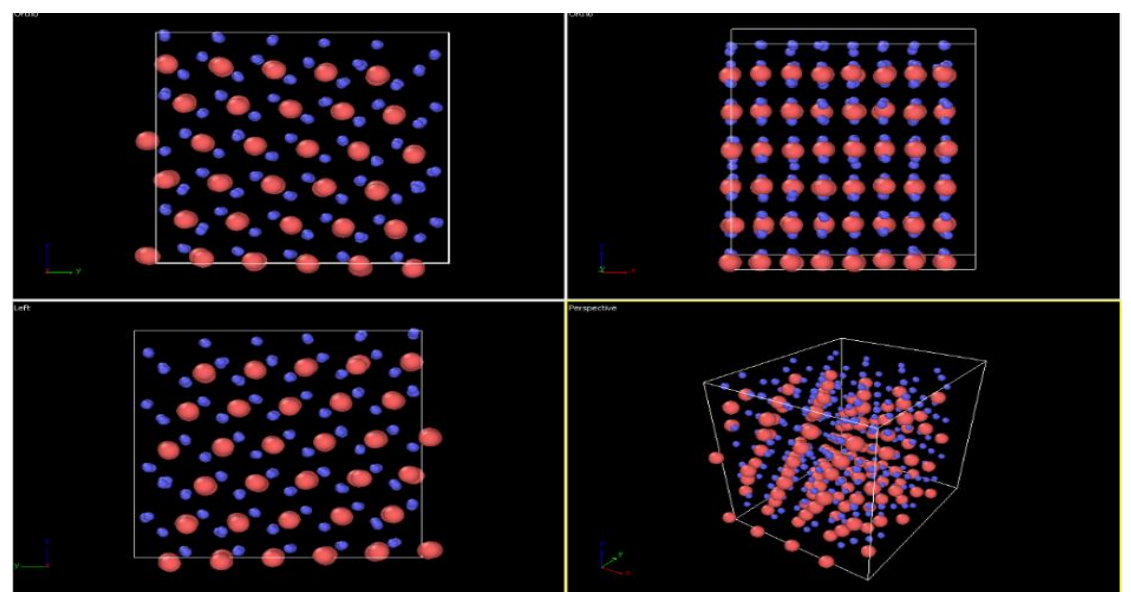

Figure 37: Hydride orientation along $\{111\}$ plane, zirconium is marked as brown, hydrogen is marked as blue. 


\subsection{Uniaxial tensile strain applied to hydride along different crystal directions}

Periodic boundary conditions (PBC) are applied in $\mathrm{x}, \mathrm{y}$ and $\mathrm{z}$ directions. The hydride structure is first relaxed using energy minimization and then is equilibrated using constant pressure and temperature NPT ensemble. The equilibrated hydride structure is studied for variation of energy with strain along different directions. A constant strain rate of $0.0001 \AA /$ picoseconds is applied on the structure. The energy of the structure is calculated at each strain value. Figure 38 indicates that with increase of strain along [ $\left[\begin{array}{lll}1 & \overline{1} & 0\end{array}\right]$ direction the energy of system is higher when compared to the energy of the system for the strain applied in [111] direction.

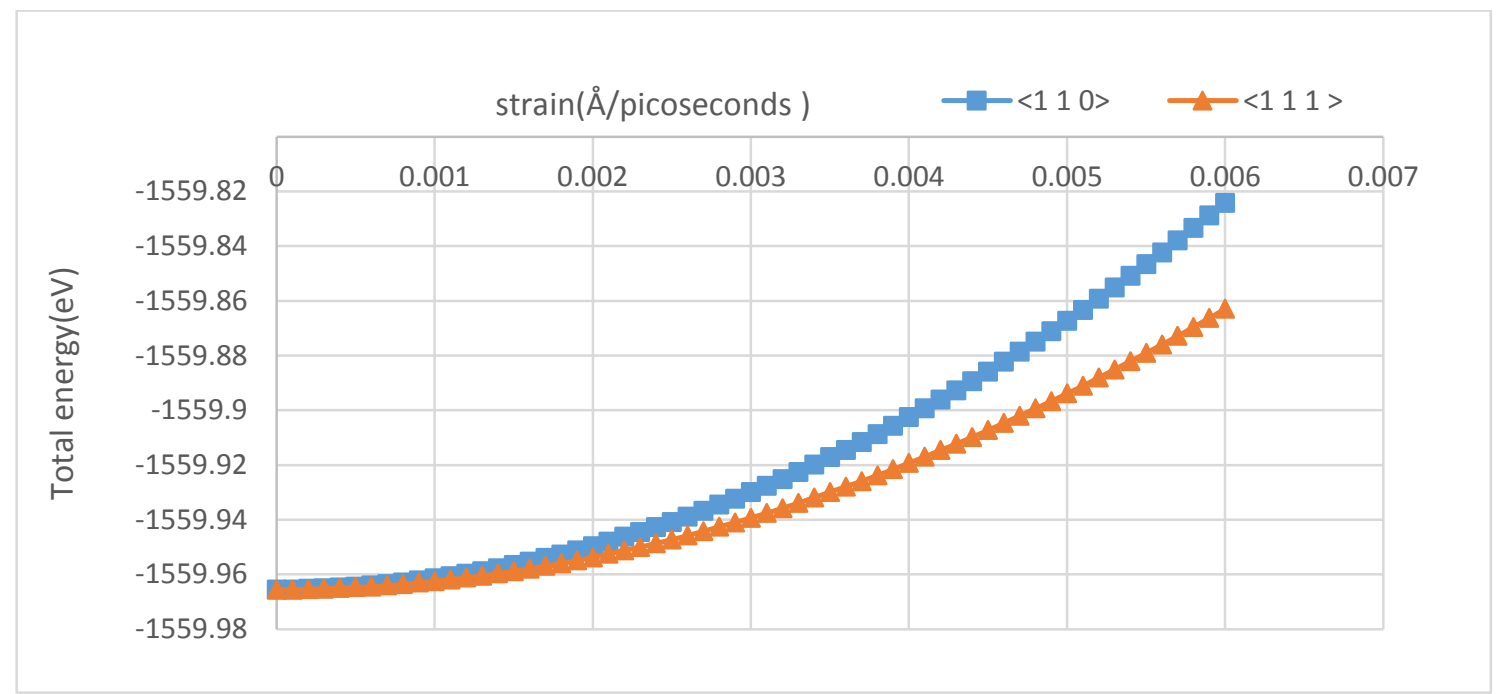

Figure 38: Energy variation with strain along [ $\left[\begin{array}{lll}1 & \overline{1} & 0\end{array}\right]$ and [111] crystal directions of hydride

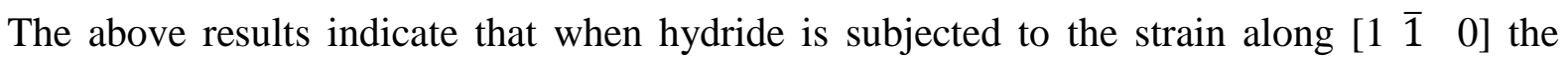
energy of the hydride is higher this energy induces hydride to reorients in direction perpendicular to application of strain which is having low energy. The graph suggests that at after $0.002\left(\AA /\right.$ picoseconds) strain hydride energy is [ [ $\left.\begin{array}{lll}1 & \overline{1} & 0\end{array}\right]$ increased in compared to [111] direction of application of strain. This theory could be the possible explanation of hydride reorientation on stress as seen by many researchers.

\subsection{Uniaxial tensile stress and strain analysis on hydride}

The hydride is analyzed for stress and strain along different crystal directions. Stress and strain is plotted as shown in figure 39. 


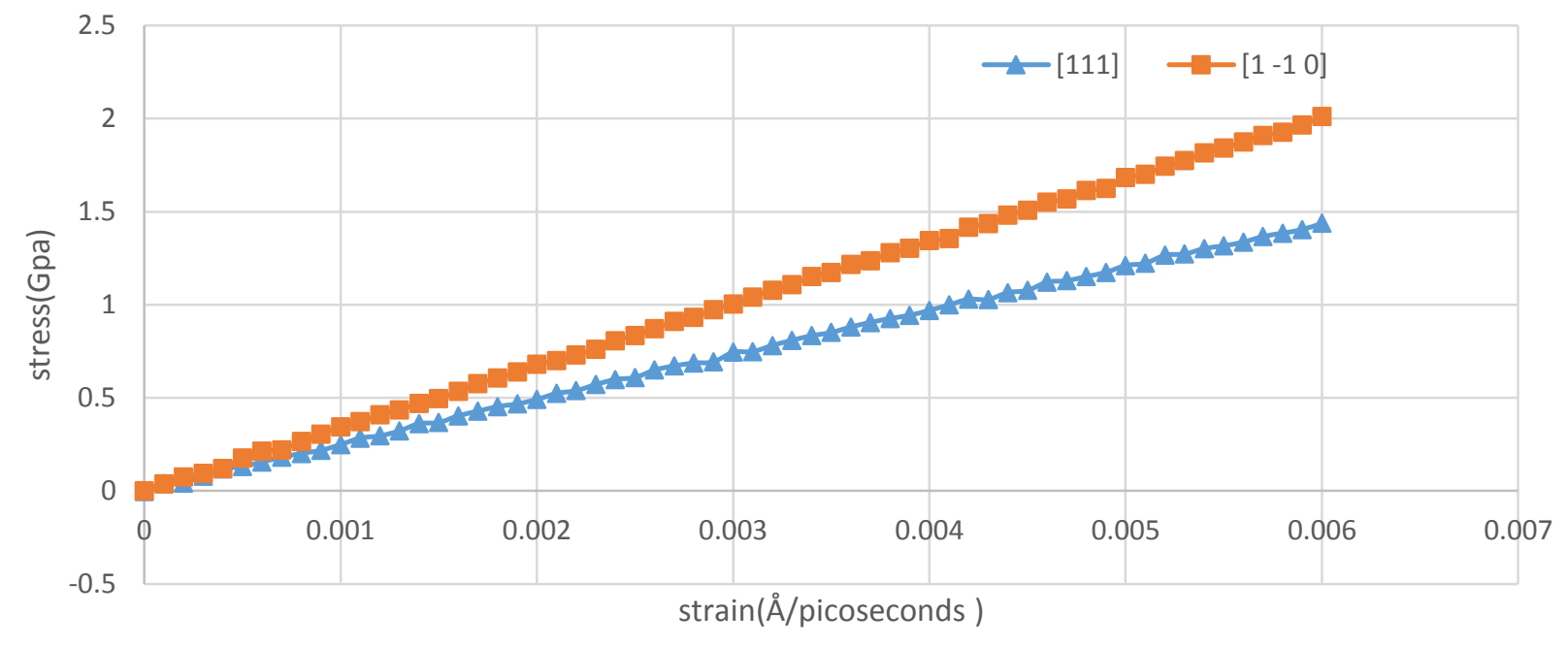

Figure 39: Stress-strain variation along [ $11 \overline{1} 0]$ and [111] crystal directions of hydride

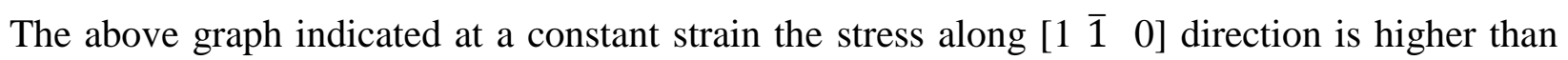
along [111] direction. High stress along [ $\left[\begin{array}{lll}1 & \overline{1} & 0\end{array}\right]$ is due high packing density of atoms when compared to [111] direction.

\subsection{Strain energy at $623 \mathrm{~K}$}

The experimental results indicate that hydride reorientation takes place at temperature $350^{\circ} \mathrm{C}$, for this reason the hydride model is tested at this temperature. A structure with $8.0 \AA * 7.0 \AA * 6.0 \AA$ with 16128 atoms with periodic boundary conditions applied on all 3 directions was build. This structure is minimized with energy minimization and equilibrated to $623 \mathrm{~K}$. A Tensile strain of $0.01 \AA /$ picoseconds is applied to this structure using NPT ensemble. The total energy of the system is calculated with the increase in strain as shown in figure 40 . With the increase in strain the difference in the energy along [ $\left[\begin{array}{lll}1 & \overline{1} & 0\end{array}\right]$ and [111] crystal directions is seen to change. This difference is energy could be responsible for hydride reorientation. 


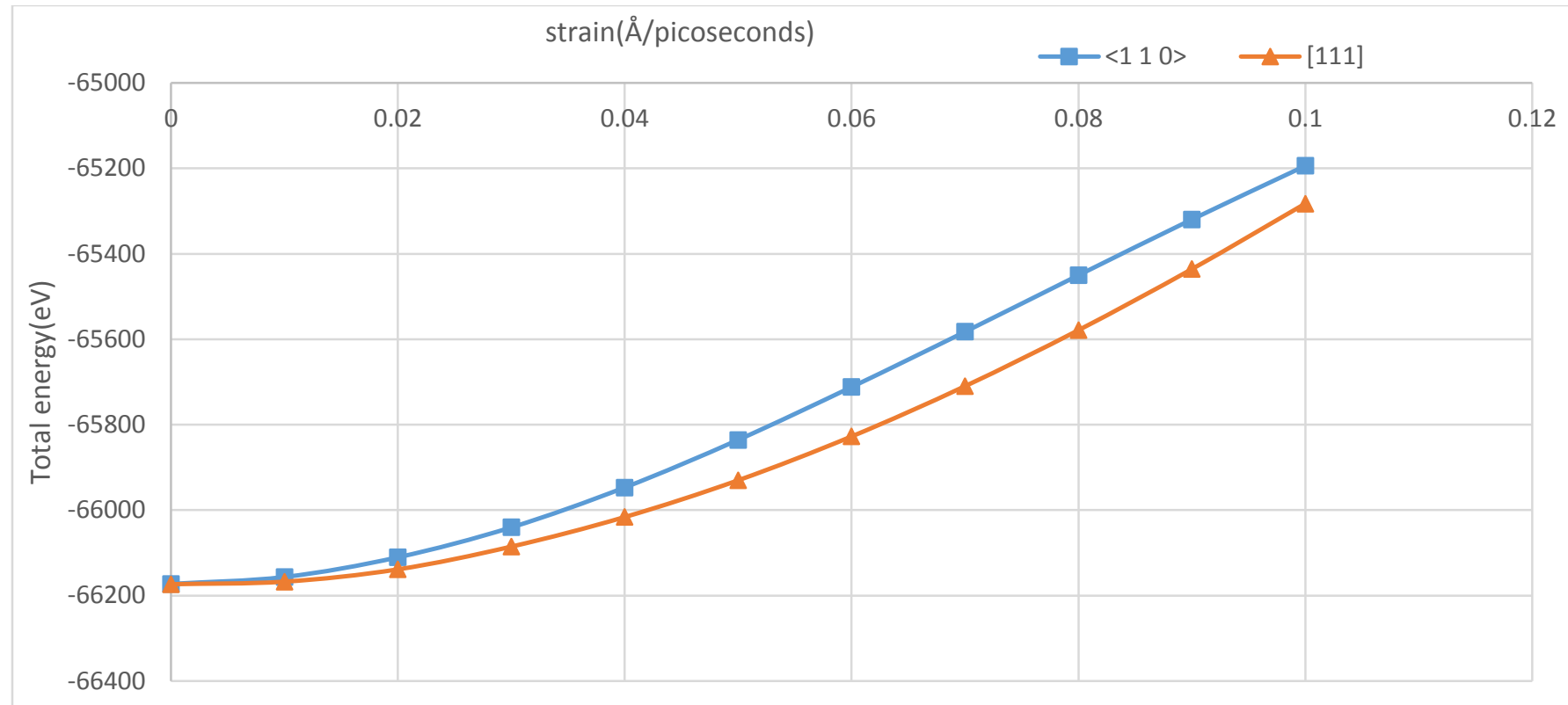

Figure 40: Energy difference in hydride along [ $\left.1 \begin{array}{lll}1 & 1 & 0\end{array}\right]$ and [111] is seen to increase with strain at $623 \mathrm{~K}$

\subsection{Conclusions}

Molecular dynamics simulations performed on hydride indicate that with increase in strain along

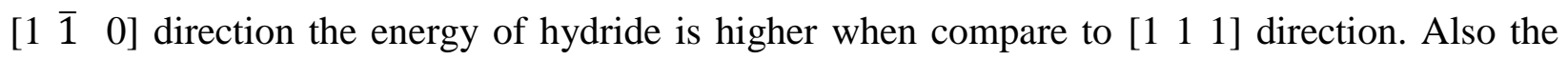
stresses along [ $\left[\begin{array}{lll}1 & 0\end{array}\right]$ is higher compared to [111] direction. The high energy and stress along [1 $\overline{1} 0]$ direction could possibly explain hydride reorientation on application of stress. 


\section{CHAPTER 8}

\section{INTERFACIAL CRACKING OF HYDRIDE IN ZIRCONIUM}

Zircaloy-4 is a key structural material of CANDU fuel-tubes. Hydrides precipitate when the

solubility limit of hydrogen in $\mathrm{Zr}$ alloys is exceeded. These hydrides precipitate at both intergranular boundaries and within the grains causing deleterious impact on the mechanical properties of the zirconium [8]. Research studies [1-3] have shown that intergranular hydrides could play more important role in hydride-induced rupture compared with intra-granular hydrides, because cracking is more likely to take place in intergranular hydrides [1]. Intergranular hydrides could cause rapid intergranular fracture under tensile stress. Experimental studies have shown that hydrides formed are mainly $\delta-\mathrm{ZrH}_{1.5}$ and were located both within the grains and along the grain boundaries. However further analysis at the grain boundaries show that they follow often the crystallographic relation $\left(\begin{array}{llll}0 & 0 & 0 & 1\end{array}\right) \alpha-\mathrm{Zr} / /\left\{\begin{array}{lll}111 & \delta & \delta \\ \mathrm{ZrH}_{1.5}\end{array}\right.$ [8]. The preferential sites for the grain boundary hydrides were basal planes of zirconium matrix located close to grain boundaries. Hydrides formed at a temperature and pressure of $350^{\circ} \mathrm{C}$ and $20 \mathrm{MPa}$ respectively with orientation relationship (0001) $\alpha-\mathrm{Zr} / /\{111\} \delta-\mathrm{ZrH}_{1.5}$ were formed under unstressed condition along radial direction of the zirconium tube. On application of hoop stress the hydrides are found to orient $\{10 \overline{1} 1\} \alpha-\mathrm{Zr} / /$ \{111) $\delta$ - $\mathrm{ZrH}_{1.5}$ relationship [52]. However this type of behavior requires study of the hydrides at the Interface of zirconium at molecular scale.

Then Objective of this work is to investigate fracture of hydrides at the interface with zirconium and to study the process of re-orientation of hydrides under stress and explain these processes at the level of molecular dynamics simulation.

\subsection{Structure of Interface (0001) $\alpha-\mathrm{Zr} / /\{111\} \delta-\mathrm{ZrH}_{2}$}

The experimental studies have shown that hydrides prefers orientation of $(0001) \alpha-\mathrm{Zr} / /\{111\} \delta$ $\mathrm{ZrH}_{1.5}$. Figure 41 shows atomic model of (0001) $\alpha-\mathrm{Zr} / /\{111\} \delta-\mathrm{ZrH}_{2}$ interface with periodic boundary conditions applied in $\mathrm{x}, \mathrm{y}$ and $\mathrm{z}$ directions. The interface consists of 9200 atoms arranged along [0001] direction of zirconium. The distance between the atoms along [0001] direction is $2.595 \AA$. The hydrogen's are placed in tetrahedral sites of hydride. 


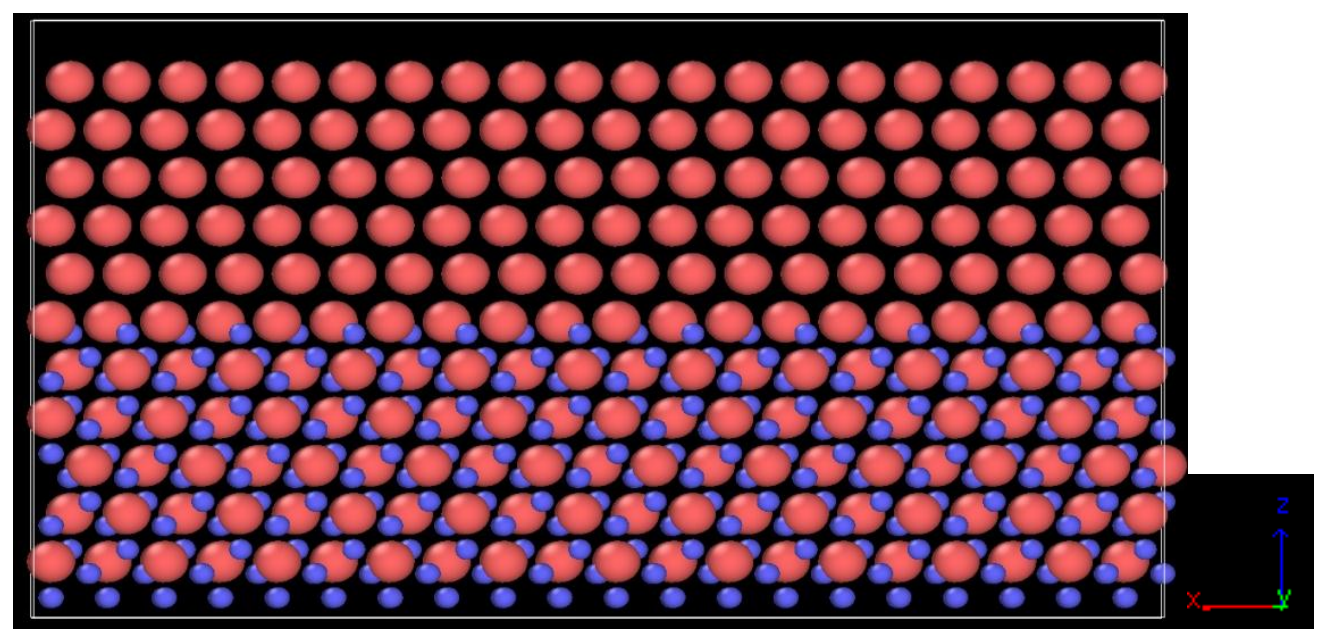

Figure 41: Atomic arrangement of interface (0001) $\alpha-\mathrm{Zr} / /\{111\} \delta-\mathrm{ZrH}_{2}$ with zirconium (brown) and hydrogen (blue) as per experimental data

\subsection{Minimized lattice structure of Interface}

The energy of structure is minimized to relax the positions of atoms at the interface. The minimized energy is used in calculating lattice parameters. The figure 42 is the relaxed atomic coordinates. The converged energy is shown in figure 43 and converged lattice parameters are shown in table 13.

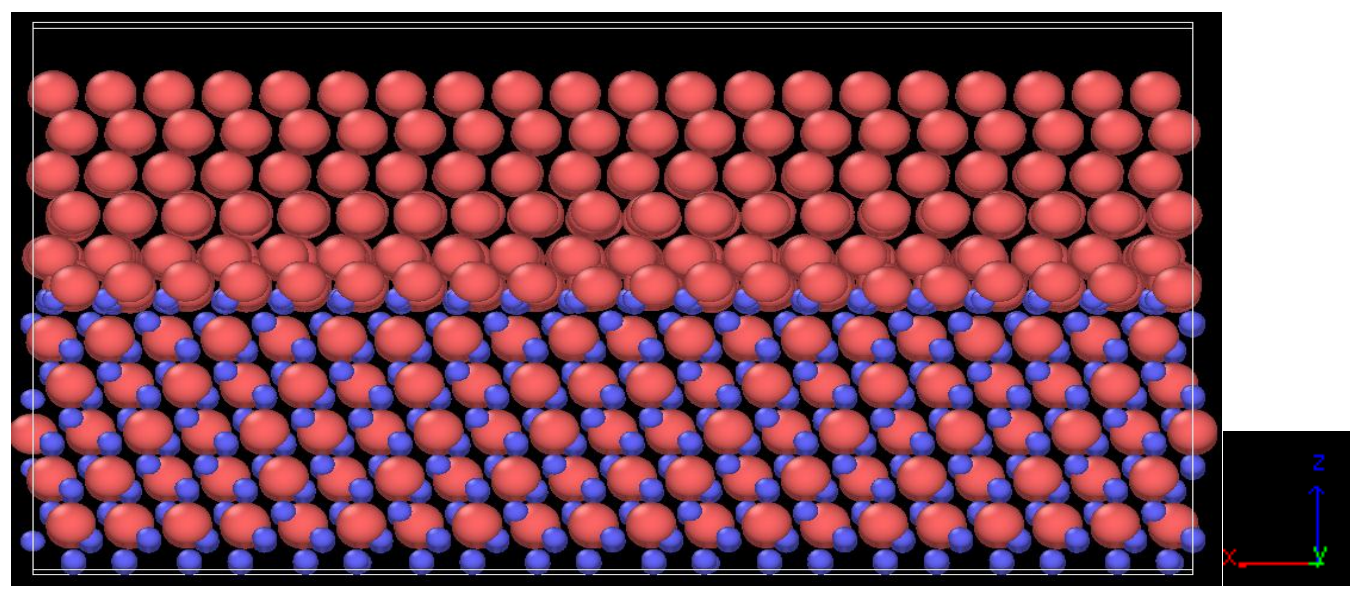

Figure 42: Relaxed Interface (0001) $\alpha-\mathrm{Zr} / /\{111\} \delta-\mathrm{ZrH}_{2}$ with zirconium (brown) and hydrogen (blue) 


\section{Minimized Energy}

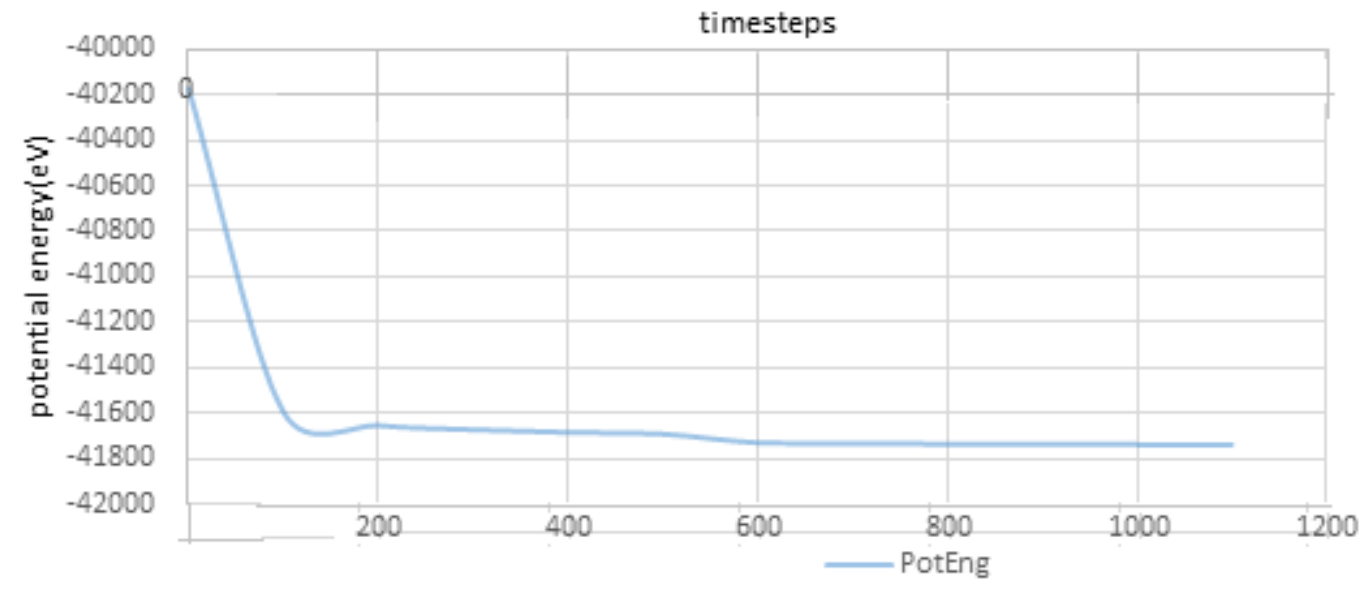

Figure 43: Energy convergence of Interface using MEAM potential

Table 13: Minimized lattice parameters for Interface

\begin{tabular}{|l|l|}
\hline Parameters & Calculated values \\
\hline $\mathrm{x}(\AA)$ & 5.826053032 \\
\hline $\mathrm{y}(\AA)$ & 6.7300343 \\
\hline $\mathrm{z}(\AA)$ & 33.080876 \\
\hline $\begin{array}{l}\text { Cohesive } \\
\text { energy(eV/atom) }\end{array}$ & -4.54700514 \\
\hline
\end{tabular}

The above parameters indicate minimized lattice parameters for the Interface. The energy of formation of this Interface is $-4.547 \mathrm{eV}$.

\subsection{Stress Analysis on the Interface of $\alpha-\mathrm{Zr} / \delta-\mathrm{ZrH}_{2}$}

The Interface is subjected to stress to investigate the crack initiation point or weakest link in the interface. Periodic boundary conditions are applied in all directions of the structure in order to maintain similar neighborhood of the atoms. The system is fully minimized to obtain minimum energy configuration. The structure is annealed to a temperature of $100 \mathrm{~K}$. The structure is then equilibrated using NPT (constant pressure and temperature) ensemble in order to maintain the required temperature to the structure. A constant strain rate of $0.01 \AA /$ picoseconds is applied to the structure with increasing timesteps. After a $40 \%$ deformation in the structure crack initiation 
takes place in brittle zirconium hydride as shown in figure 44 and then it propagates into the zirconium matrix as shown in figure 45.The simulations observed were in good agreement with experimental observation as shown in figure 46.

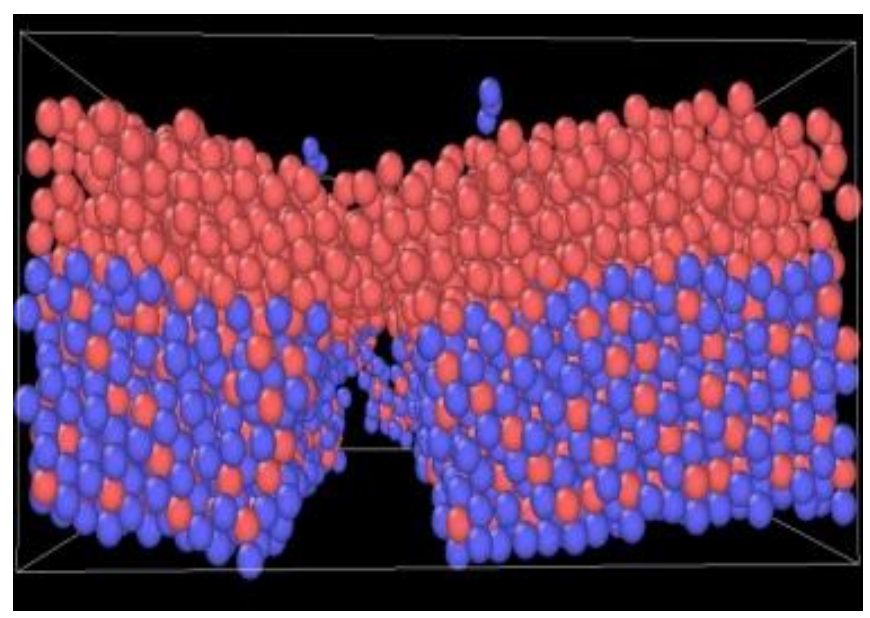

Figure 44: Crack initiation hydride

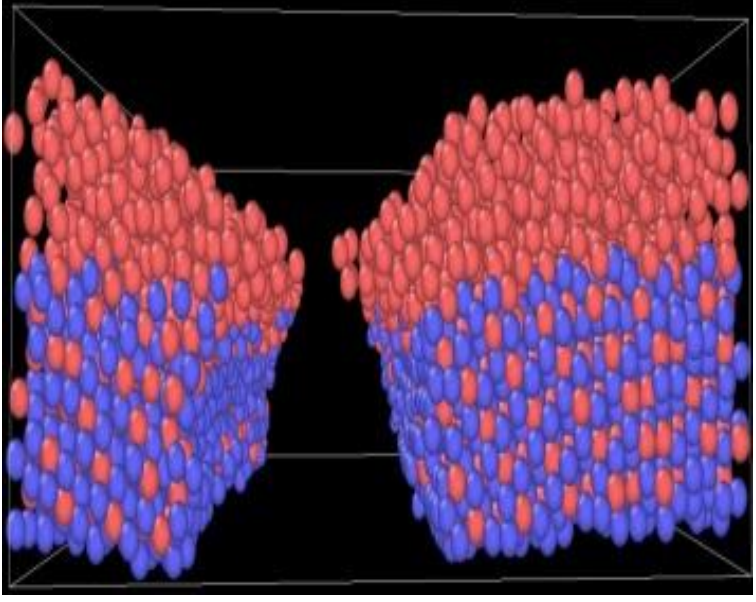

Figure 45: crack propagation from hydride Into zirconium matrix

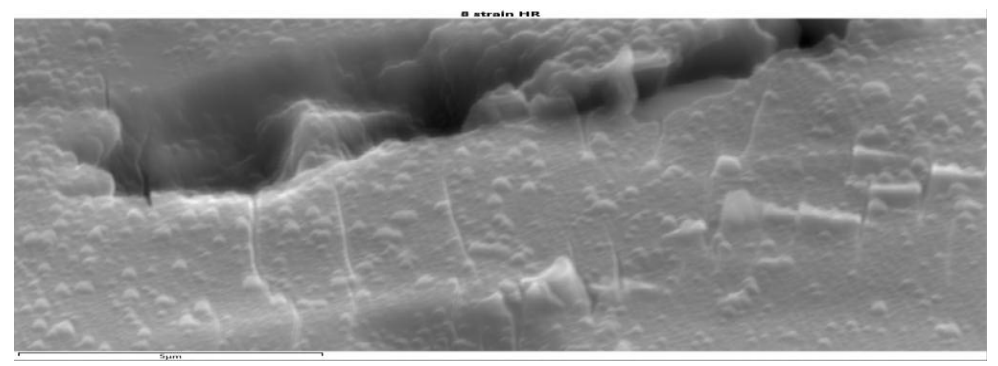

Figure 46: N.A.P. Kiran Kumar, J. A. Szpunar, SEM image small cracks developing in $\delta-\mathrm{ZrH}_{2}$ and propagating into zirconium matrix

\subsection{Stress analysis on Interface at various operating conditions}

Further analysis is done at various operating conditions in order to study the effect of temperature. Figure 47 shows stress and strain at various temperatures indicate that with increase in temperature crack initiation occurs early and the material fractures with lower strain rate. 


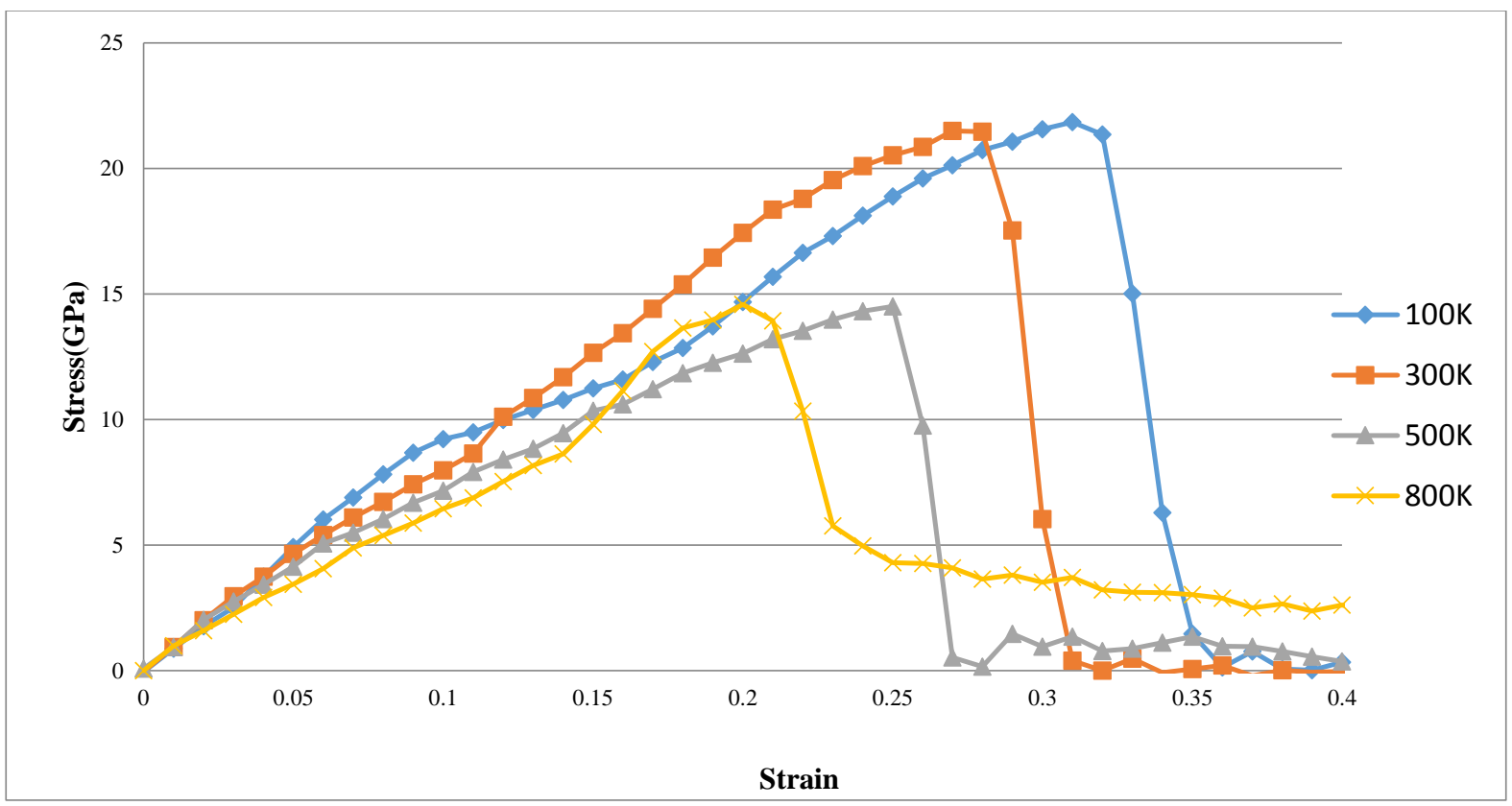

Figure 47: Increase in temperature nucleates crack early in Interface

\subsection{Conclusions}

- Interface (0001) $\alpha-\mathrm{Zr} / /\{111\} \delta-\mathrm{ZrH}_{2}$ is modeled and energy minimized.

- The cohesive energy of the interface is calculated as $-4.547 \mathrm{eV} /$ atom

- Increase stress show crack nucleation in hydride and this crack propagates into zirconium matrix.

- Increase of temperature shows early nucleation of crack causing fracture of zirconium tubes

- Good agreement with experimental observation as is seen validates the results of cracking. 


\section{CHAPTER 9}

\section{CRACK PROPAGATION IN ZIRCONIUM}

Molecular dynamics can be a powerful tool for investigating atomic scale mechanical behavior at a micro-defect front. Kotrechko et al [54] and Huang et al [55] investigated the fracture process of nano-crystalline materials under axial tension. Kitamura et al [56] and Ikeda et al [57] investigated the fracture behavior of different materials and proposed the fracture criterion, which can be used to estimate the conditions of crack propagation. They described the crack growth mechanism at the front of various micro-defects and the fracture process in both BCC and FCC materials. Yet, relatively less work has been carried out on the hexagonal close-packed (HCP) materials.

Cracks and voids in zirconium are the most common defects that could be formed during manufacturing process or during operating conditions in the reactor. Hydrogen ingress into zirconium at higher concentrations generates hydrides. The effect of these hydrides on embrittlement of zirconium alloys has been studied extensively. However, even low concentrations of hydrogen in zirconium have significant influence on crack formation and propagation. The role of the hydrogen on crack propagation in zirconium is yet to be better understood. Lower concentrations of hydrogen also make the metals brittle. The brittle vs. ductile response of crystalline materials is mainly controlled by the competition between crack growth and the mobility of dislocations near the crack tip. The three slip systems of zirconium are (0001) [1 $\overline{2} 10]$, (0001) [11 20$]$ and (0001) [ $\overline{2} 110]$, as shown figure 48 .

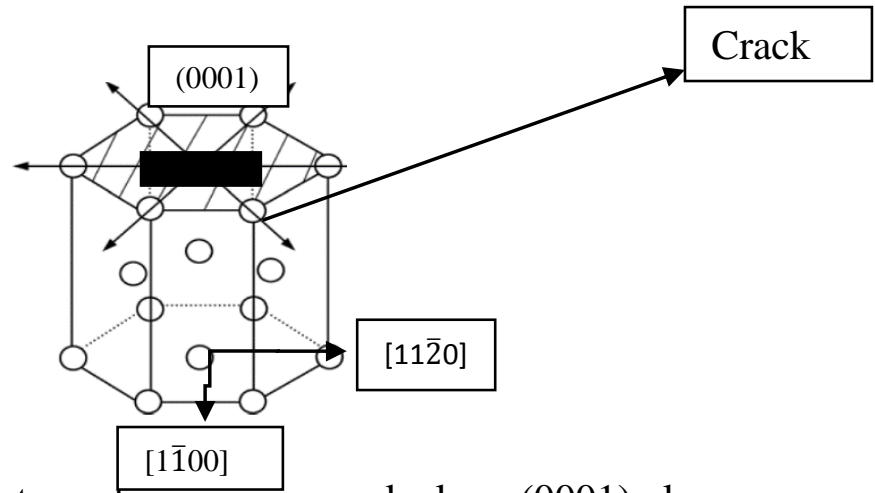

Figure 48: Crystal structure of Zirconium crack along (0001) plane 


\subsection{Bond Angle Analysis}

Bond angle analysis is performed in OVITO using the output from LAMMPS to analyze the local atomic environment of each atom according to the Auckland-Jones bond angle method [58]. This method not only differentiates the type of structure formed like fcc, bcc and hcp but also identifies dislocation defects in structures.

\subsection{Crack propagation in zirconium at different temperatures and timesteps}

\subsection{Temperature 0.01K}

Zirconium of dimension $100 \AA * 40 \AA * 3 \AA$ is setup and a crack of dimension $10 \AA * 1 \AA * 3 \AA$ is placed in zirconium as shown in figure 47 . The systems consist of 48417 atoms. The nonperiodic conditions are used in $\mathrm{x}$ and $\mathrm{y}$ directions and periodic boundary conditions are used along $\mathrm{z}$ direction. The system is fully minimized using energy minimization. The system is equilibrated to $0.01 \mathrm{~K}$. A strain rate of $0.8 \AA$ A/picoseconds is applied along y-direction to study the crack propagation.

From the bond angle analysis we can see that the atoms near the crack are disordered before any deformation strain is applied on the structure as shown in figure 49. The white atoms show the weak bonds and they are surrounding the crack.

At 0 time steps

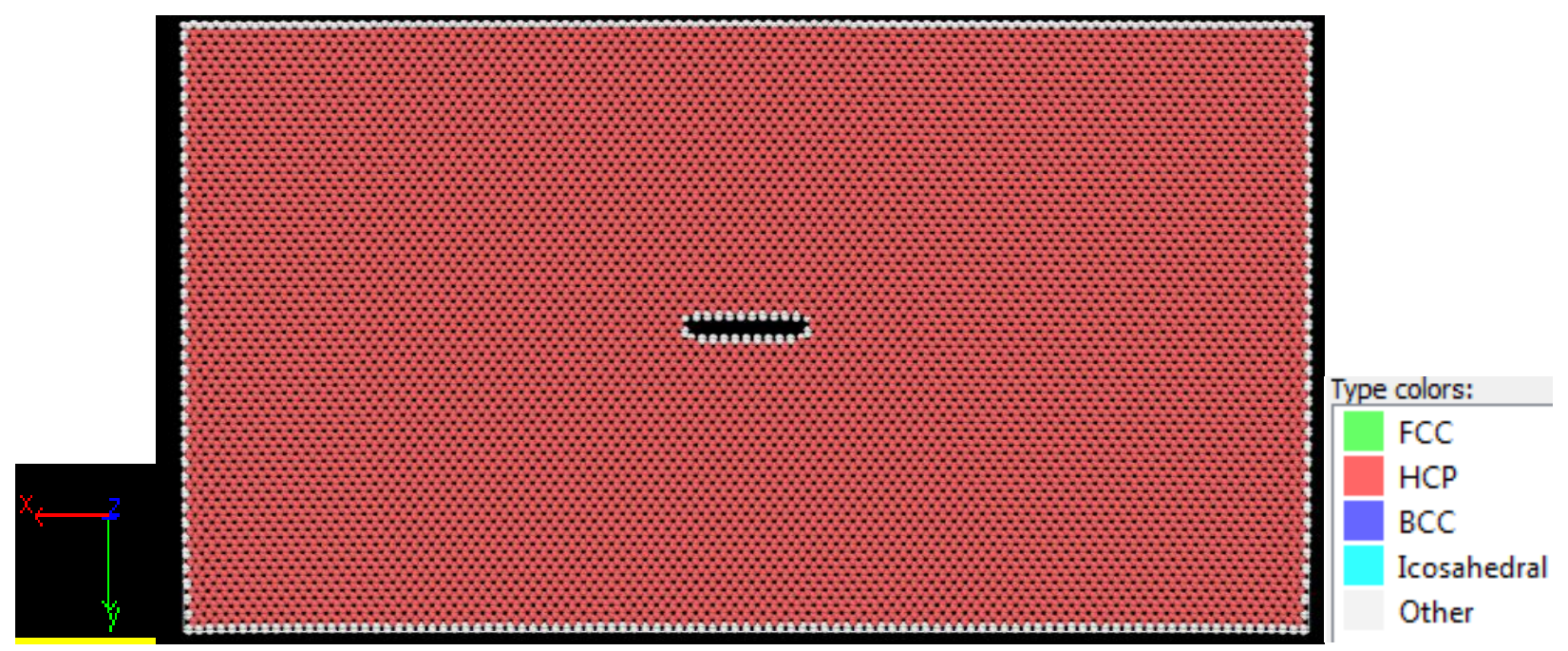

Figure 49: Shows crack inside $\alpha-Z r$ along (0001) plane 
At 100 time steps

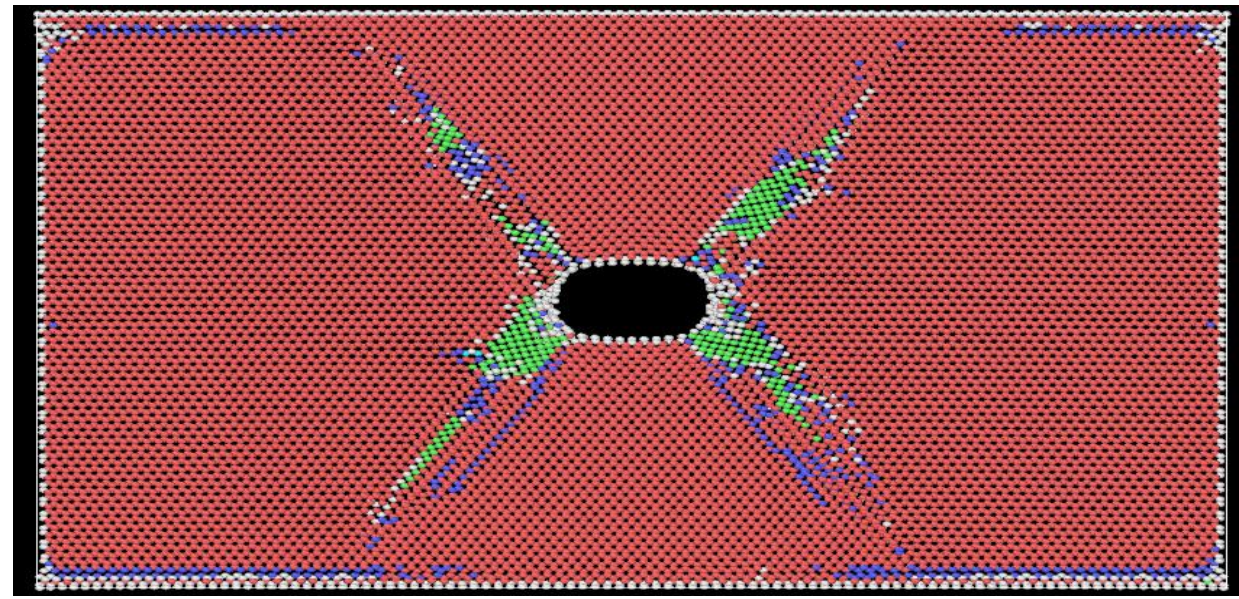

Direction of Strain applied

Figure 50: Crack propagation inside $\alpha-\mathrm{Zr}$ after 100 time steps timestep

The blue color indicates formation of $\mathrm{BCC}$ bonds formed ahead of crack propagation which could be due to emission of dislocations in direction of crack propagation as shown in figure 50. Molecular dynamics predict change in bonds surrounding the crack and crack blunting with increase in stain. As the crack propagates further weaker bonds represented by white atoms. The similar crack blunting is seen in zircaloy-4 as shown by Bertolino et al [59].

\subsubsection{In presence of hydrogen $(1 \%)$ randomly distributed}

500 hydrogen (blue) atoms are randomly distributed in zirconium as shown in figure 51. The same system is set up as above but now with hydrogen atoms inside pure zirconium. The total number of atoms in the system is 48916 atoms. The system is minimized using energy minimization. Most of hydrogen occupies tetrahedral sites as shown figure 51 . The non-periodic conditions are used in $\mathrm{x}$ and $\mathrm{y}$ directions and periodic conditions are used along $\mathrm{z}$ direction. The system is fully minimized using energy minimization . The system is equilibrated to $0.01 \mathrm{~K}$. A similar strain rate of $0.8 \AA /$ picoseconds is applied along $\mathrm{y}$-direction to study the crack propagation. 
At 0 time steps

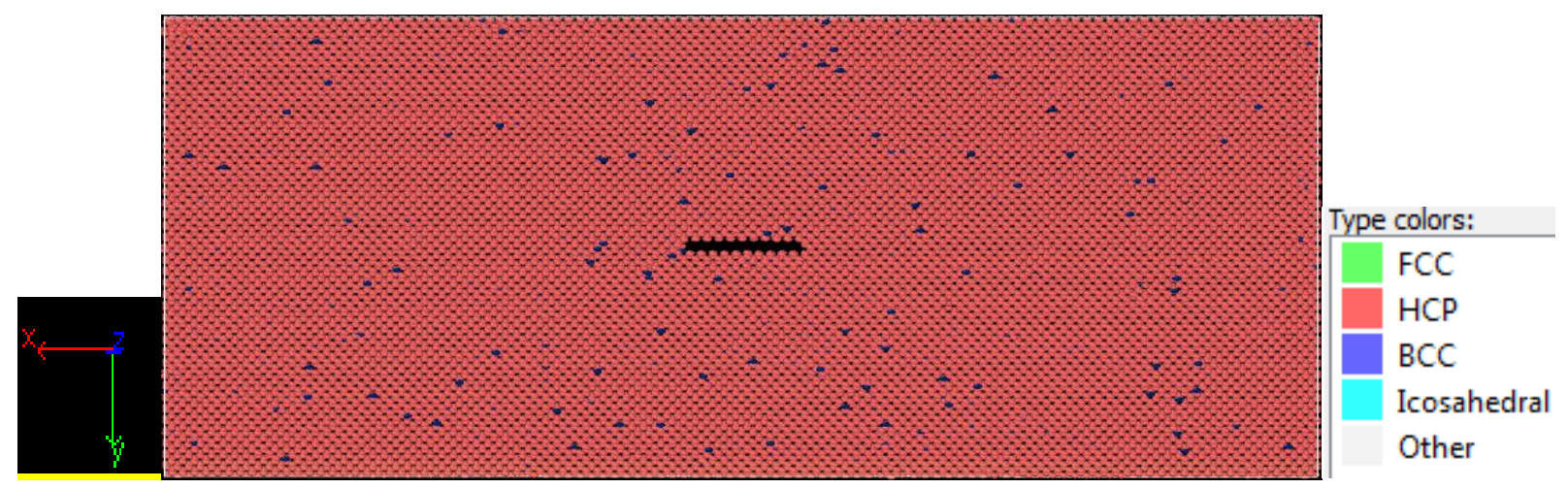

Figure 51 Crack inside $\alpha-\mathrm{Zr}$ and with randomly distributed hydrogen atoms (blue)

Before applying the tensile strain in y direction the bonds surrounding each atom in the system are observed. The sites where hydrogen atoms are present form weaker bonds thereby reduce the strength of zirconium. Now tensile strain is applied in y direction to study the crack propagation as shown in figure 52.

At 0 time steps

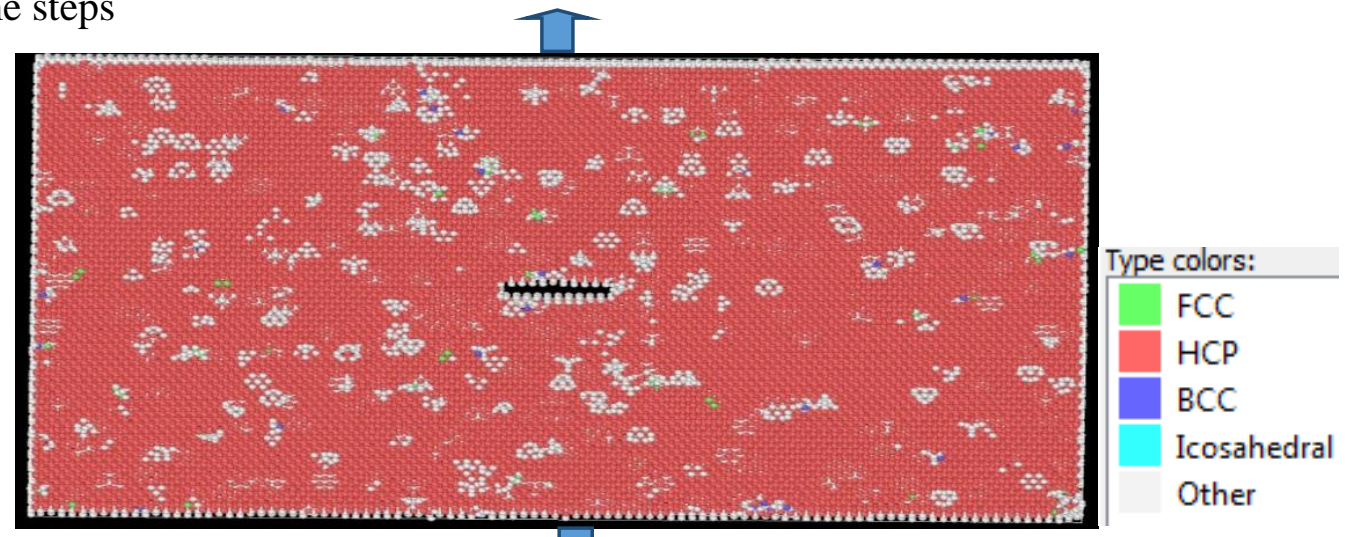

Figure 52: Weaker bonds surrounding hydrogen atoms (white color) inside $\alpha-\mathrm{Zr}$

At 25 time steps

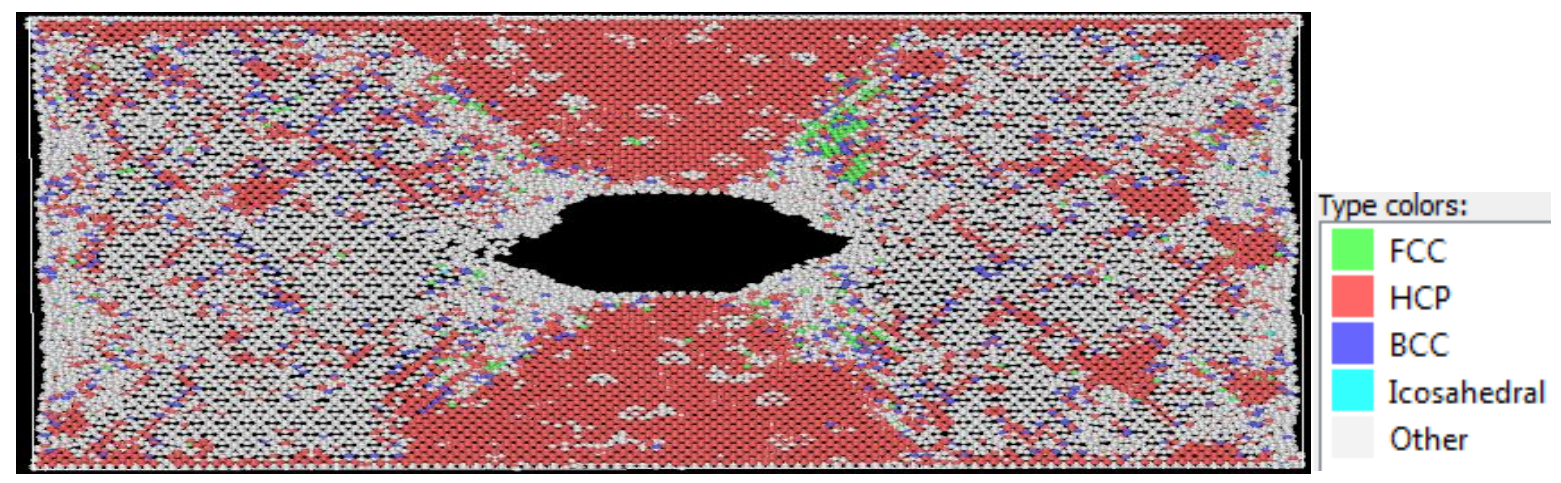

Figure 53: Crack propagation in presence of hydrogen inside $\alpha-\mathrm{Zr}$ at 25 time steps 
Figure 53 shows brittle crack propagation in zirconium due to presence of hydrogen atoms inside zirconium as compared to no hydrogen in zirconium in figure 50. The area ahead of the crack shows weaker bonds which allows crack to propagate easily. The brittle fracture is propagated throughout the zirconium structure and finally the material fails as shown in figure 54. The similar effect is observed through experiments by Beritolio et al [59] where hydrogen makes zircaloy -4 brittle and causes crack to propagate.

At 50 time steps

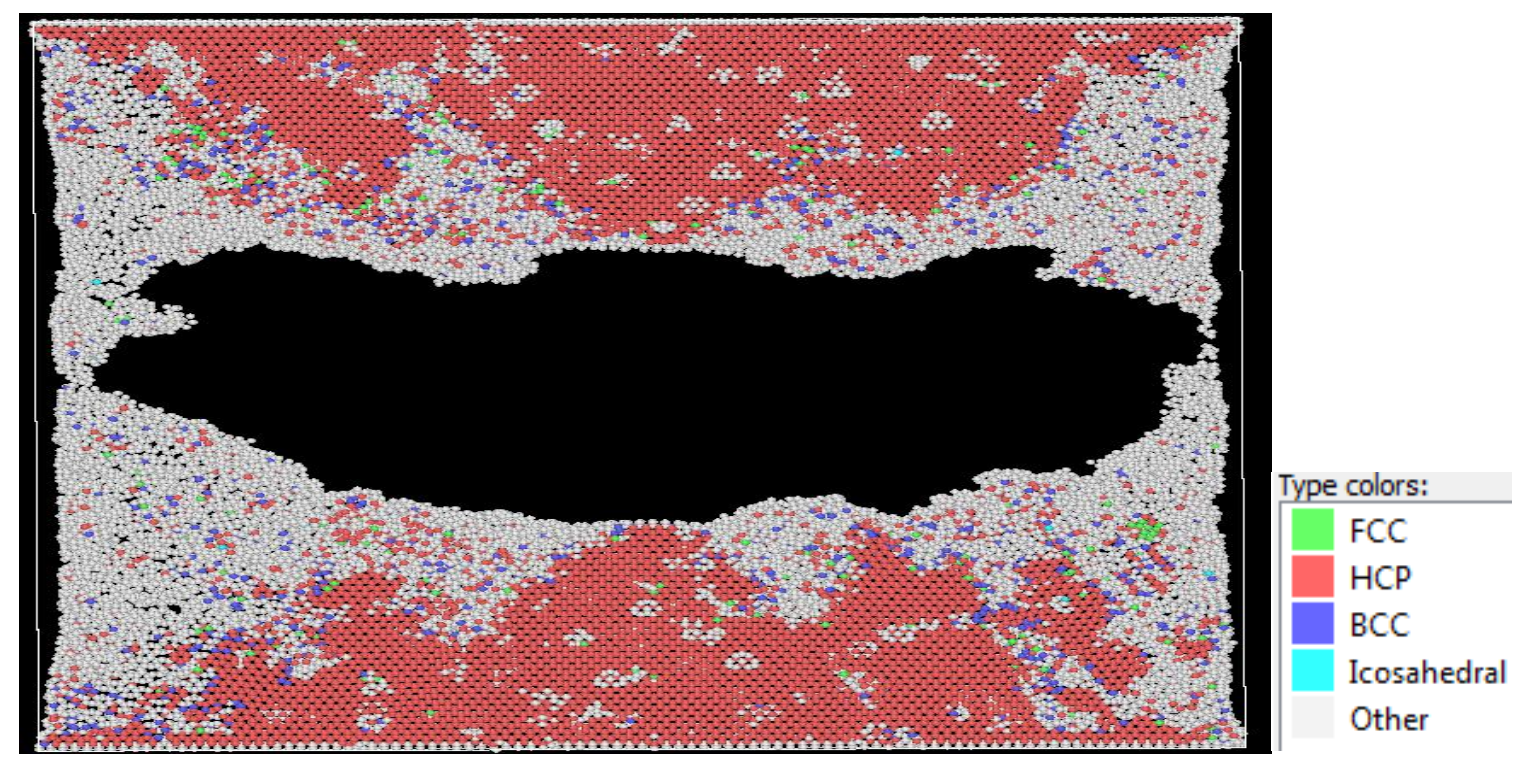

Figure 54: Crack propagation in the presence of hydrogen in $\alpha-\mathrm{Zr}$ at 50 time steps fracturing of zirconium

\subsection{Temperature $300 \mathrm{~K}$}

As we know that nuclear reactors operate at high temperatures, it is important to study the mechanism of crack propagation at higher temperatures. The system is setup for zirconium with the crack and is equilibrated to $300 \mathrm{~K}$. A velocity of $0.8 \AA$ /picoseconds in y-direction is applied to study the crack propagation at constant temperature of $300 \mathrm{~K}$. At $300 \mathrm{~K}$ after 100 time steps, the large crack propagation is seen in zirconium and as the crack propagates it branches out as shown in figure 55. Large numbers of white atoms are formed indicating weaker bonds forming thereby reducing the strength of zirconium. 
At 100 time steps

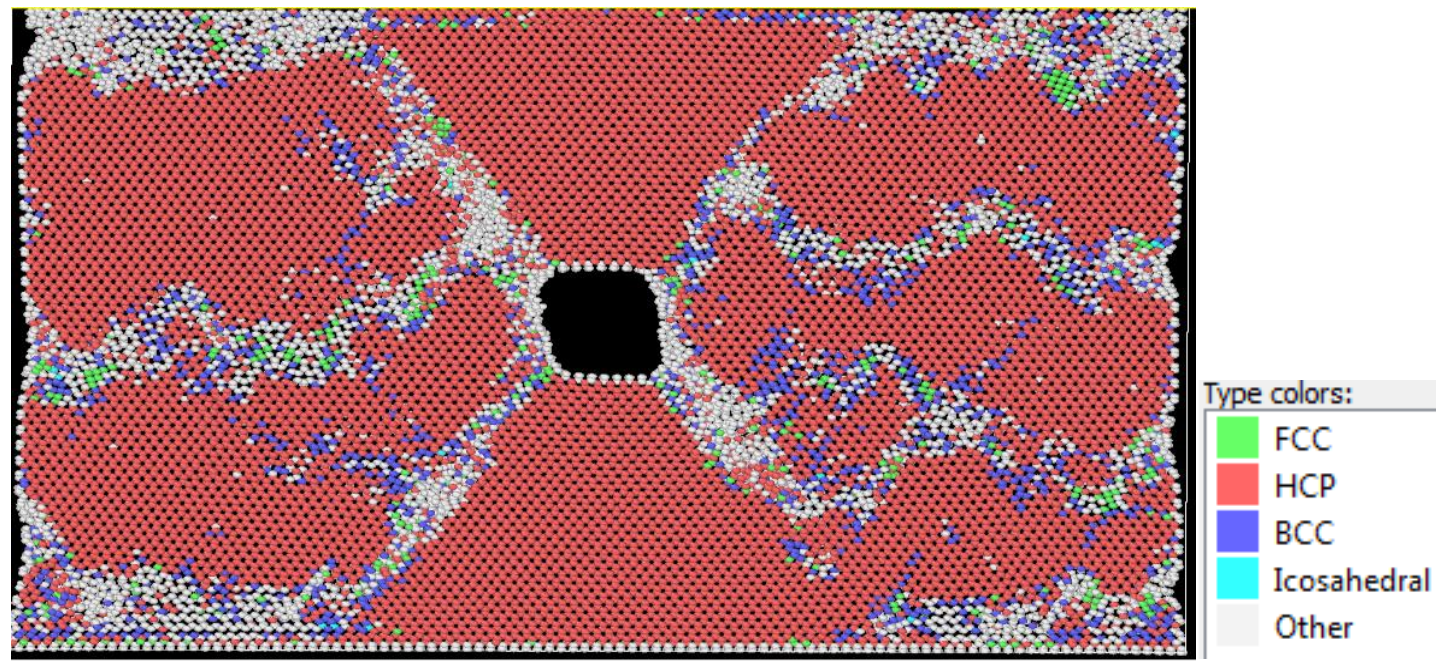

Figure 55: Crack Propagation and branching in zirconium at 300K

\subsubsection{Crack propagation in zirconium and hydrogen $(1 \%)$ at $300 \mathrm{~K}$}

At $300 \mathrm{~K}$ temperature zirconium is tested for crack propagation in presence of hydrogen and is compared to pure zirconium in figure 56. The effect of hydrogen in zirconium is analyzed after 50 timesteps of deformation.

At 50 time steps

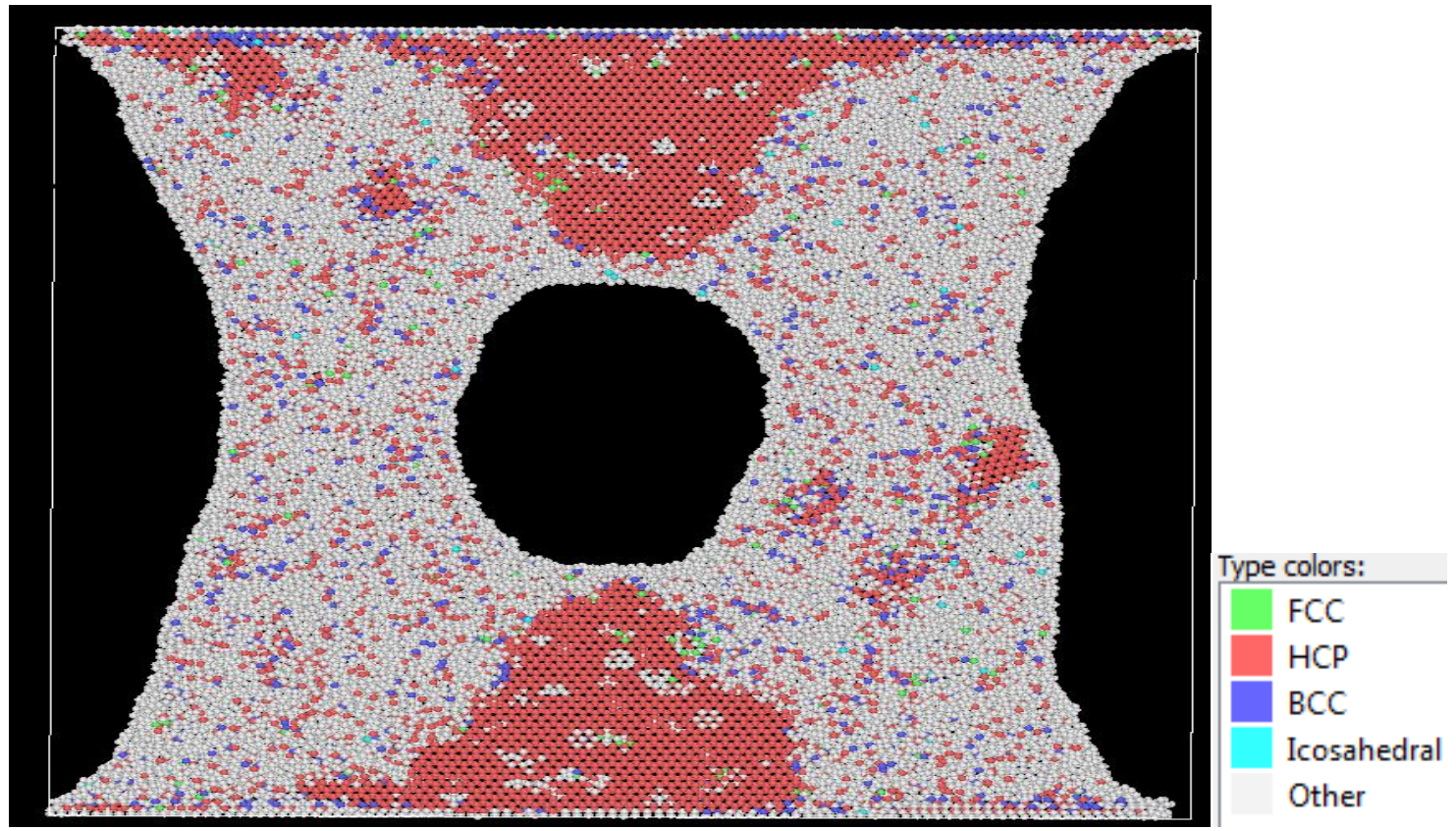

Figure 56: Crack propagation and branching in zirconium in presence of hydrogen at $300 \mathrm{~K}$ 
Due to presence of hydrogen inside zirconium and with a temperature of $300 \mathrm{~K}$ the crack blunting takes place as seen figure 54. The dislocation propagation could be restricted due to hydrogen and the material is deformed heavily. The bonds are broken, and other weaker bonds are formed reducing the strength of the zirconium.

\subsection{Temperature 500K}

The system is now setup for temperature of $500 \mathrm{~K}$ for pure zirconium. Higher branching and faster propagation of crack is seen in figure 57. Crack propagation and blunting is seen to be higher in figure 55 than the system with $300 \mathrm{~K}$.

At 100 time steps

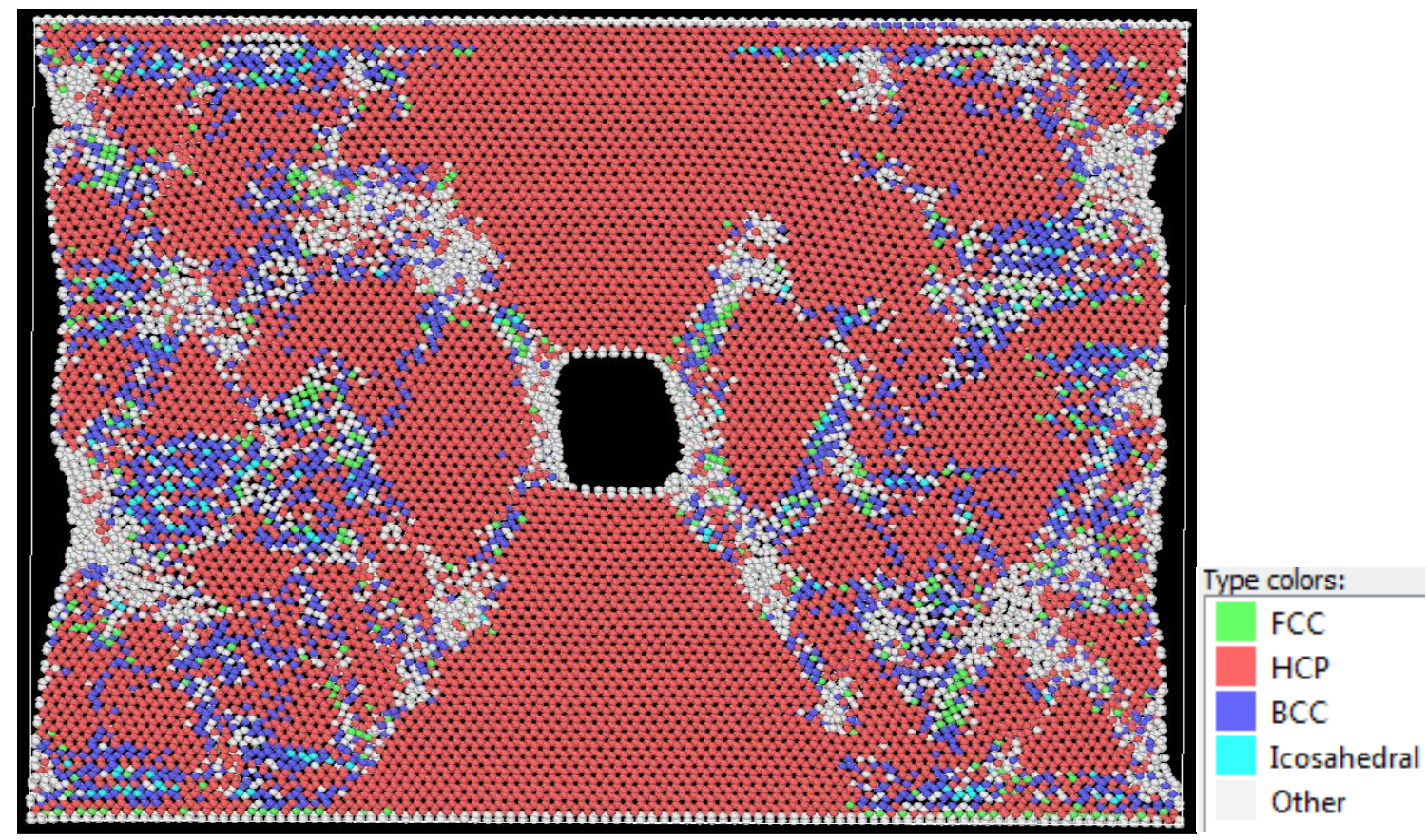

Figure 57: Crack propagation and branching in zirconium at 500K

\subsubsection{Crack propagation in zirconium and hydrogen $(1 \%)$ at $500 \mathrm{~K}$}

At $500 \mathrm{~K}$ in presence of hydrogen zirconium the effect is studied .As the temperature increases the hydrogen diffusion is higher as seen in chapter 6 and the material is more heavily deformed compared to the system without hydrogen. The crack elongation and blunting is seen in figure 58. The bonds are readily broken making the material to lose strength more than in the system without hydrogen. 


\section{At 50 time steps}

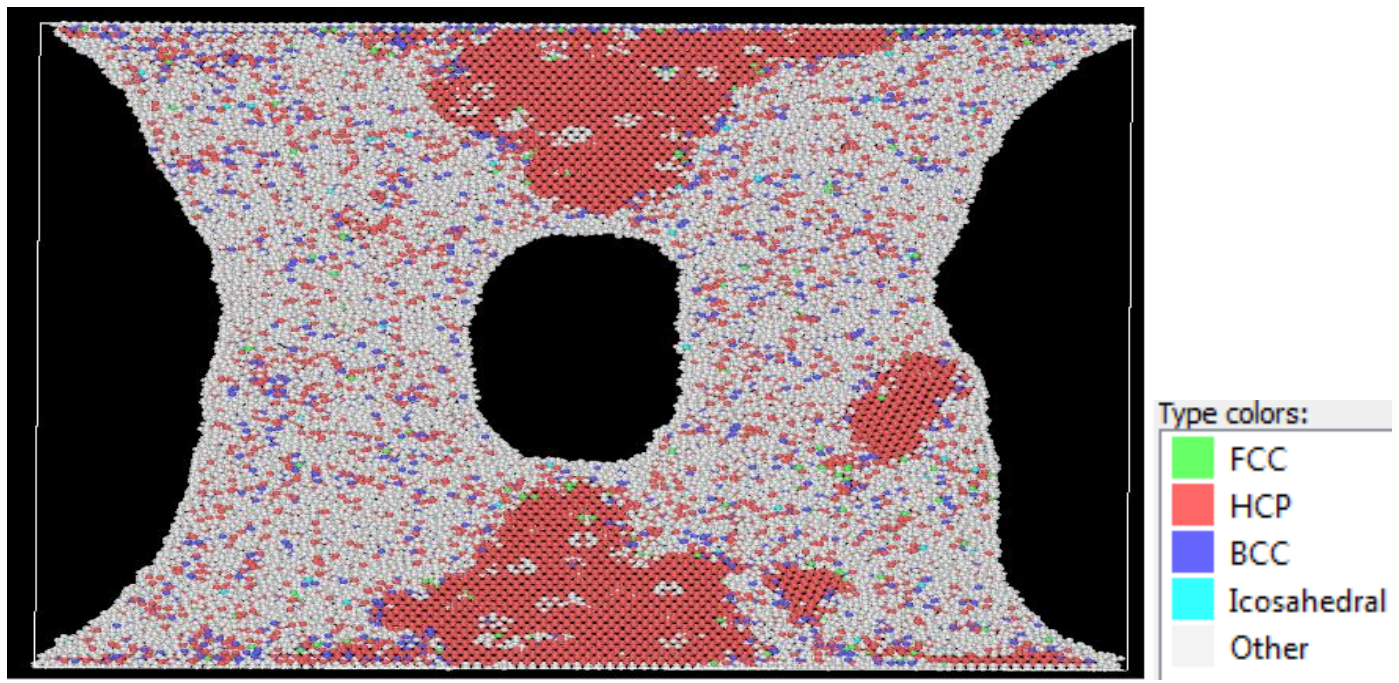

Figure 58: Crack propagation and branching in zirconium in presence of hydrogen at 500K

\subsection{Temperature $800 \mathrm{~K}$}

Further the zirconium now is equilibrated to higher temperatures of $800 \mathrm{~K}$. The temperature affected the area around the crack. Cracks are seen nucleate and material is much more heavily deformed compared to lower temperature. The area around the crack is completely deformed. The shape of the crack is seen to change as seen in figure 59.

At 100 time steps

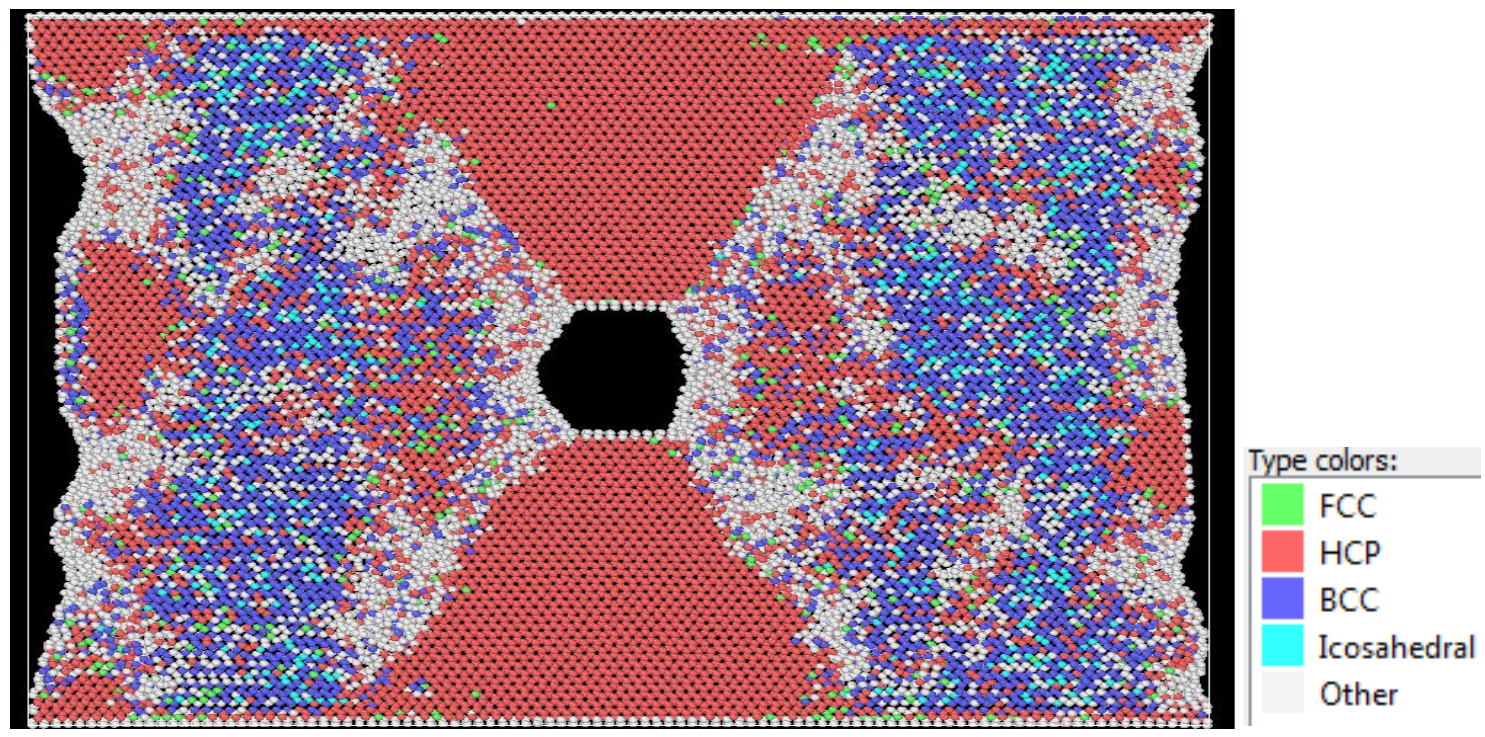

Figure 59: Crack propagation and branching in zirconium at 500K 


\subsubsection{Crack propagation in zirconium and hydrogen $(1 \%)$ at $800 \mathrm{~K}$}

Effect of hydrogen on the crack at $800 \mathrm{~K}$ is studied. The crack blunting is higher in the direction of pull. At higher temperatures hydrogen completely diffusion is much higher in zirconium and makes zirconium loses strength as seen in figure 60 . The elongation of the crack increases with increase in temperature.

\section{At 50 time steps}

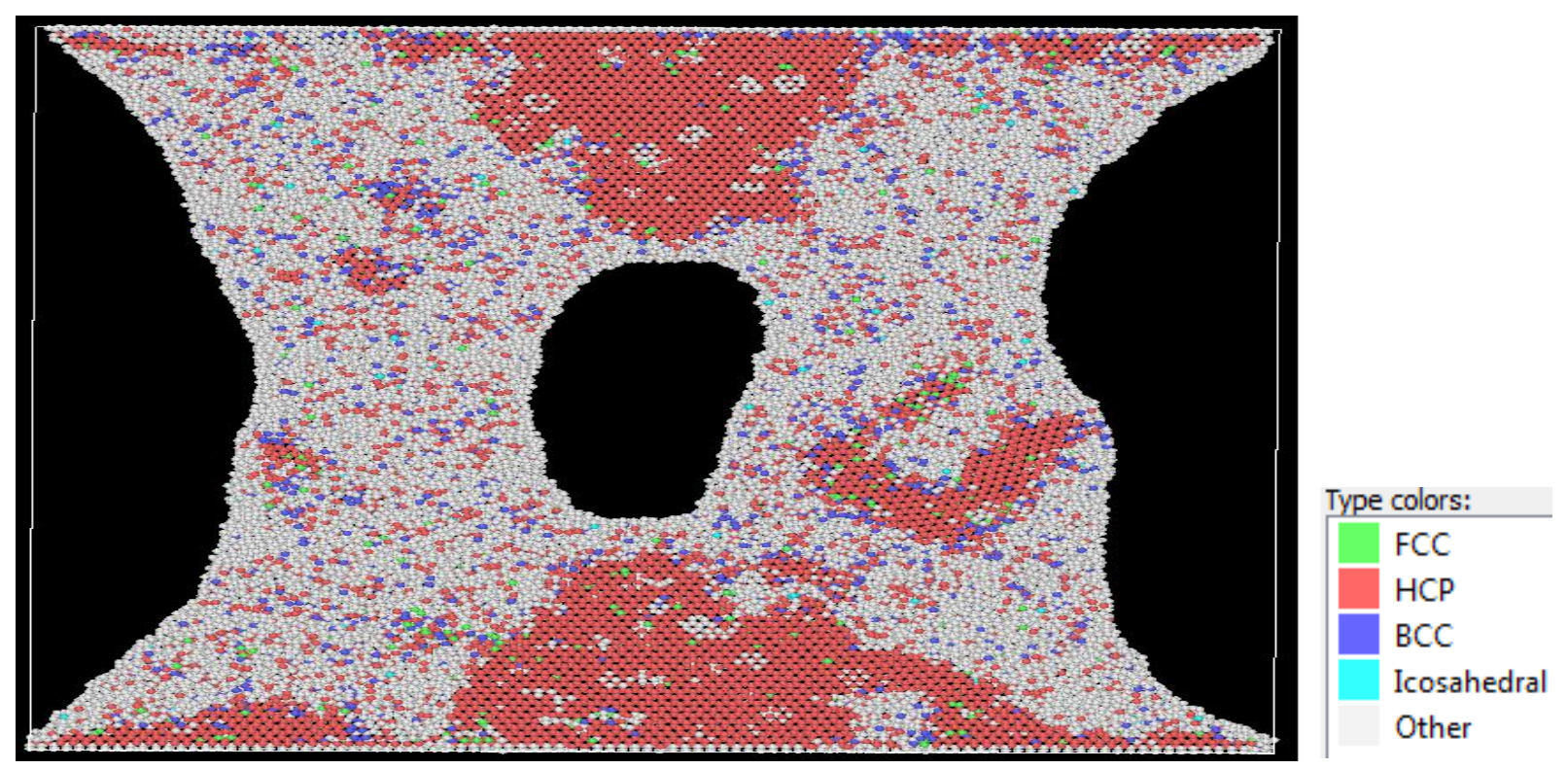

Figure 60: Crack propagation and branching in zirconium at $800 \mathrm{~K}$

\subsection{Study of stress concentration in $\alpha-\mathrm{Zr}$ in presence of hydrogen using VMD software}

A much more detailed study of stress analysis on surrounding the atom upon crack propagation is presented in this research. Stress analysis gives effect of stress on crack propagation in zirconium in the absence and presence of hydrogen at atomistic level and at the temperatures from $300 \mathrm{~K}$ to $800 \mathrm{~K}$. The output of LAMMPS is used in VMD software to study stress concentration surrounding the atoms.

\subsubsection{At 0.01K}

The figures below show stress concentration surrounding an atom when the strain loading is applied to zirconium structure in presence of the crack as seen in figure 61 


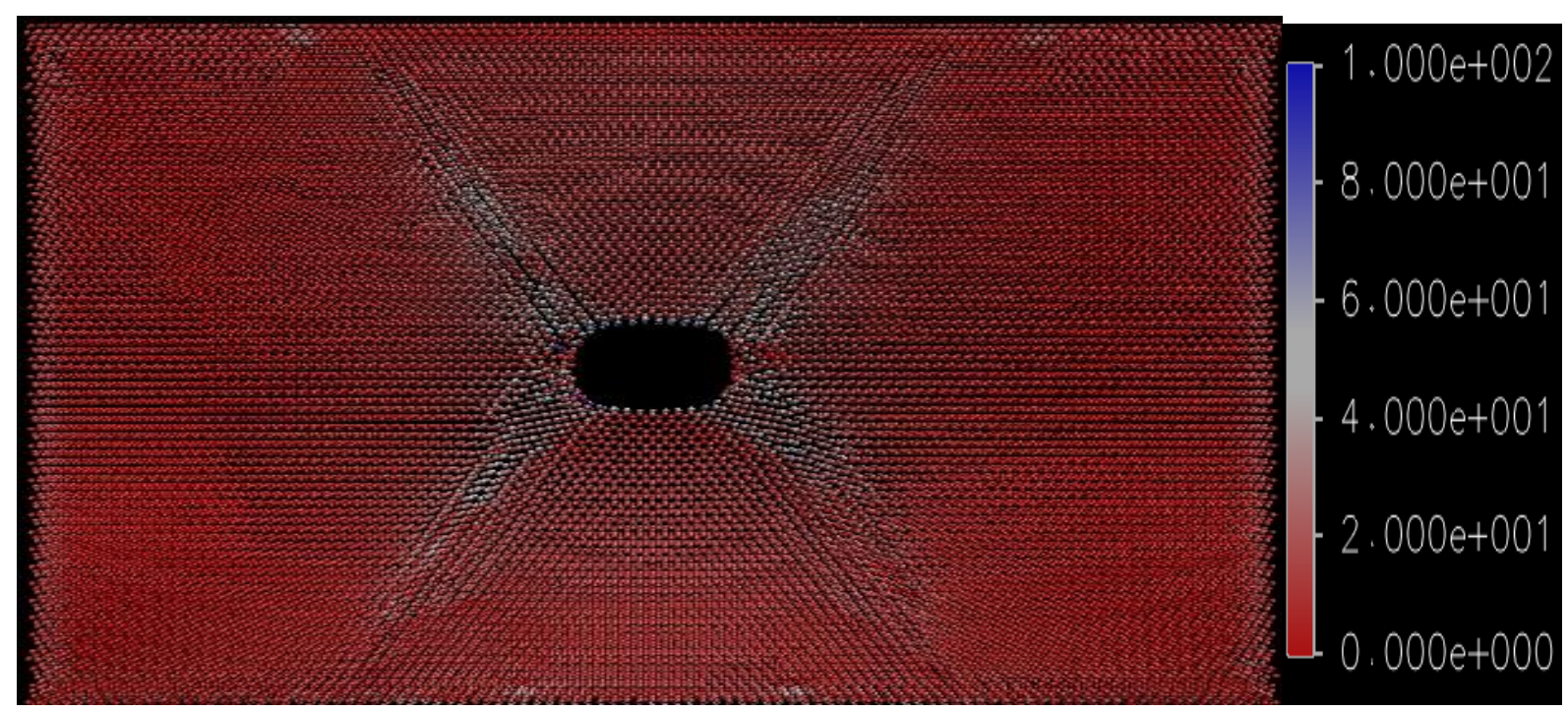

Figure 61: High stresses are seen ahead of crack causing crack to propagate in pure $\alpha-\mathrm{Zr}$

\section{$\underline{\text { With Hydrogen }}$}

Higher stresses are seen in zirconium in presence of hydrogen causing crack to propagate and material finally fractures as seen in figure 62.

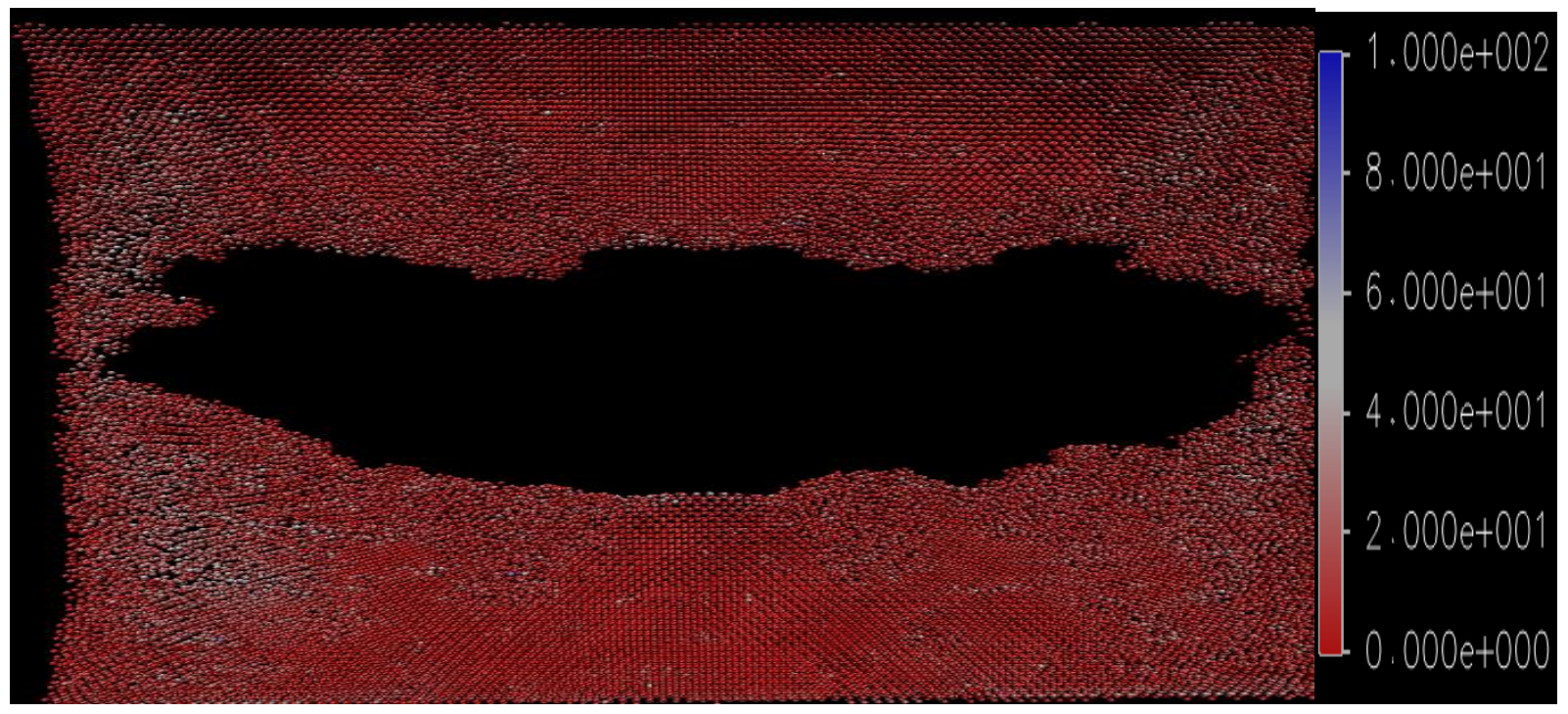

Figure 62: Crack propagation and failure of $\alpha \mathrm{Zr}$ is observed due to presence of hydrogen

\subsubsection{At 300K}

At $300 \mathrm{~K}$ the crack is seen to branch and propagate with higher stresses as seen in figure 63 


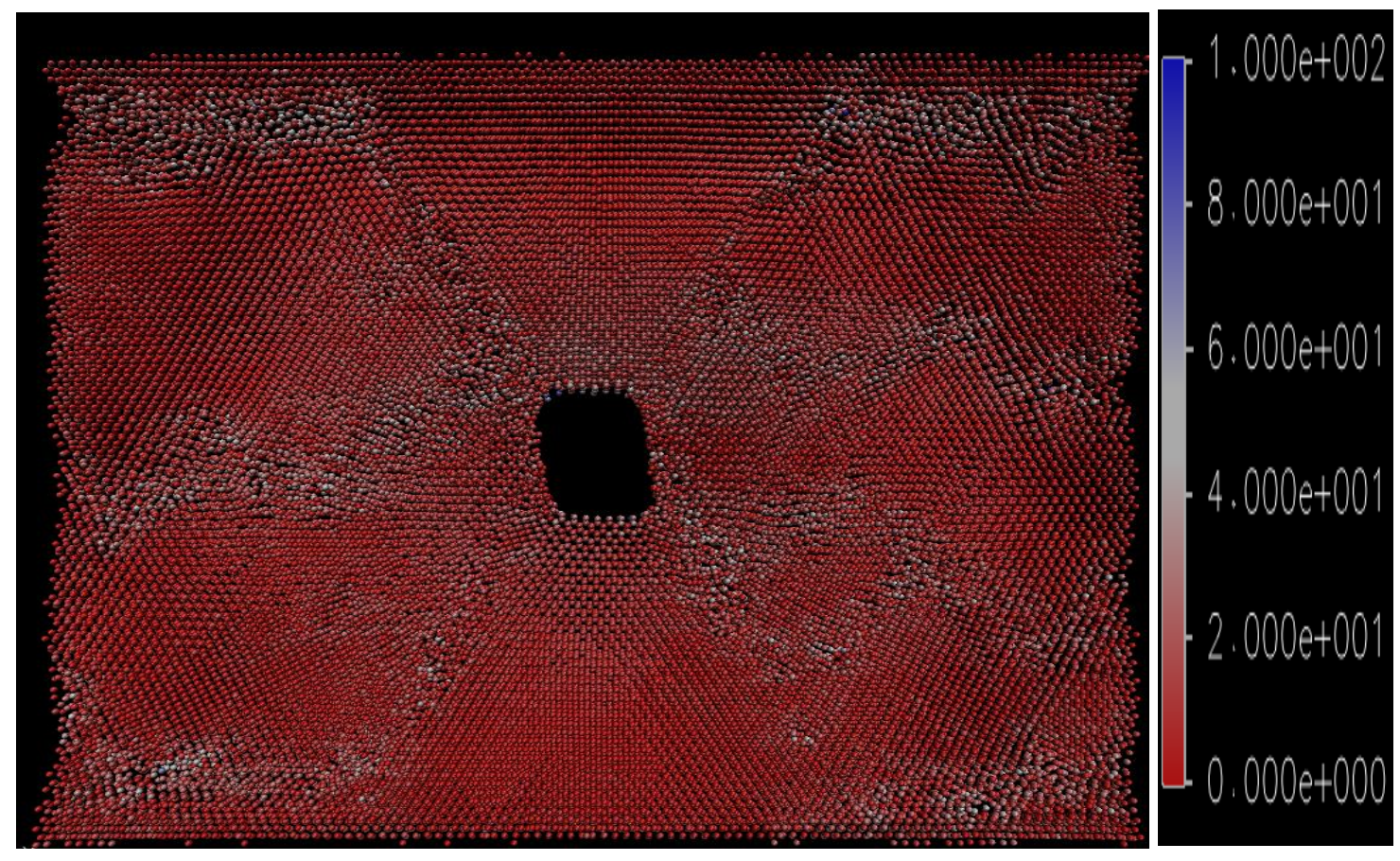

Figure 63: Cracks propagation and branching at a temperature of $300 \mathrm{~K}$.

\section{With Hydrogen}

Blunting of crack is seen in presence of hydrogen and high stresses are recorded surrounding the crack at $300 \mathrm{~K}$ as seen in figure 64 .

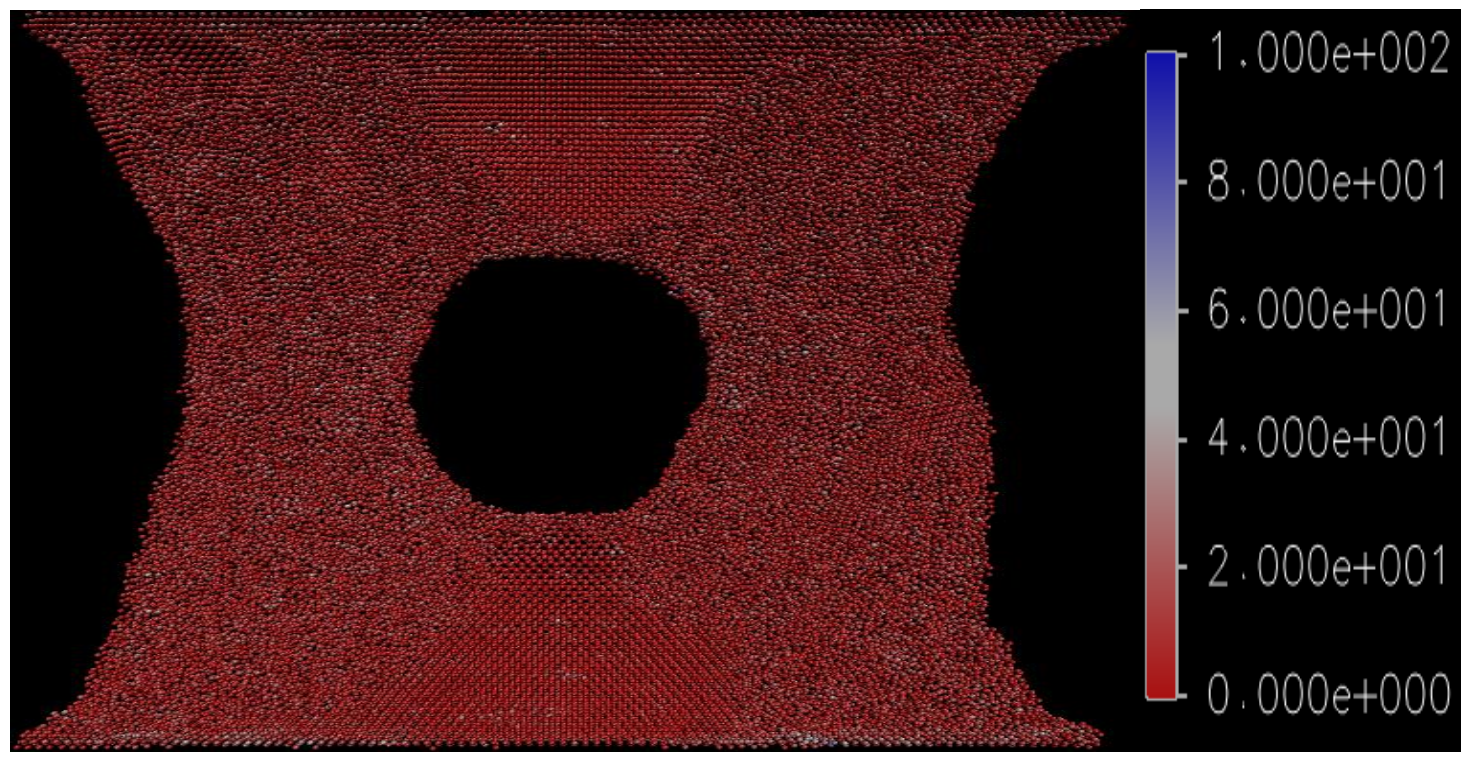

Figure 64: High crack blunting of crack in presence of hydrogen at 300K 


\subsubsection{At 500K}

At $500 \mathrm{~K}$ high stresses are seen in direction of crack propagation as seen in figure 65

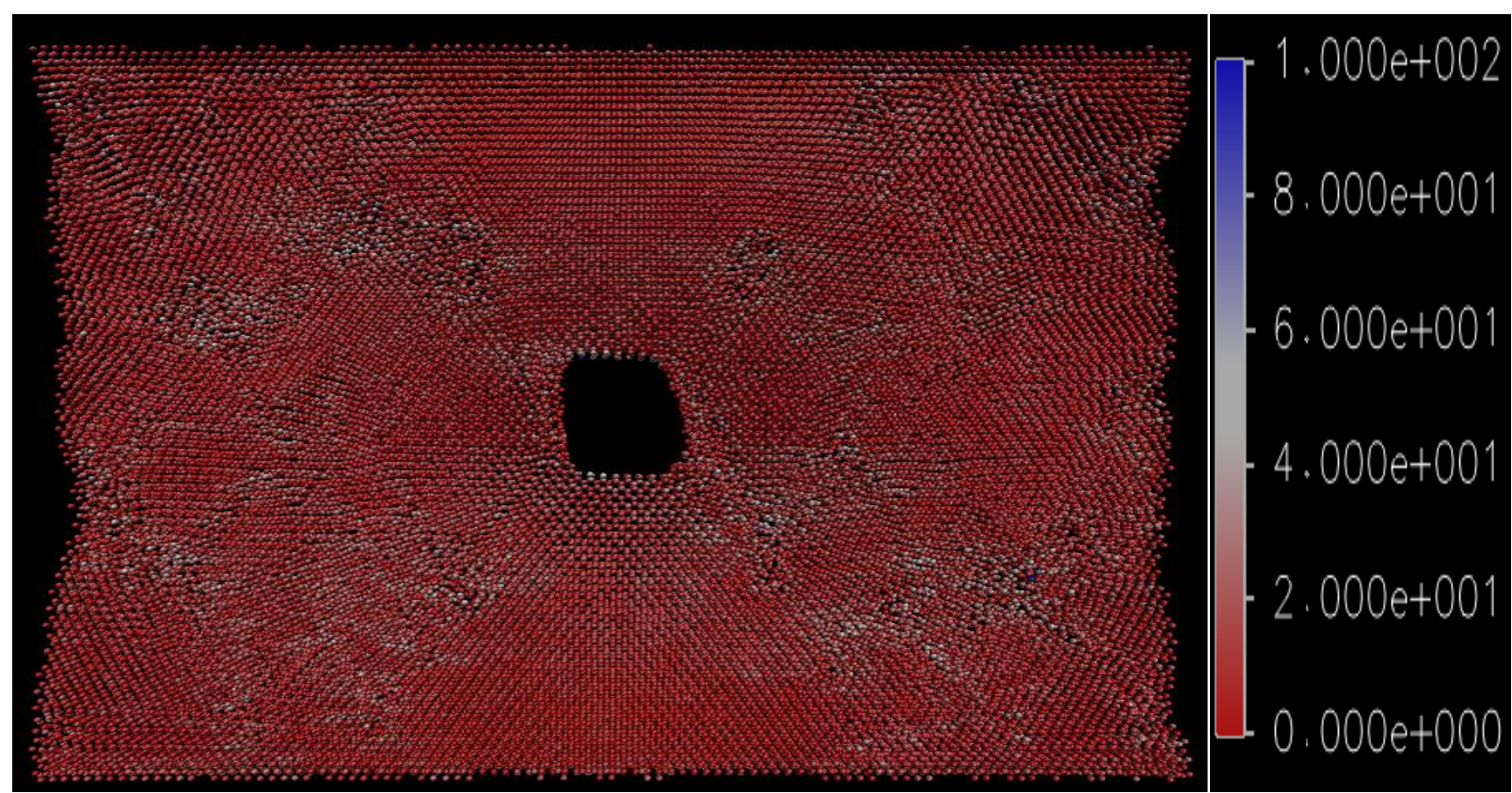

Figure 65: High stresses surrounding crack and also ahead of crack at 500K

\section{$\underline{\text { With Hydrogen }}$}

At $500 \mathrm{~K}$ high stresses with crack blunting are seen in surrounding the crack propagation as seen in figure 66.

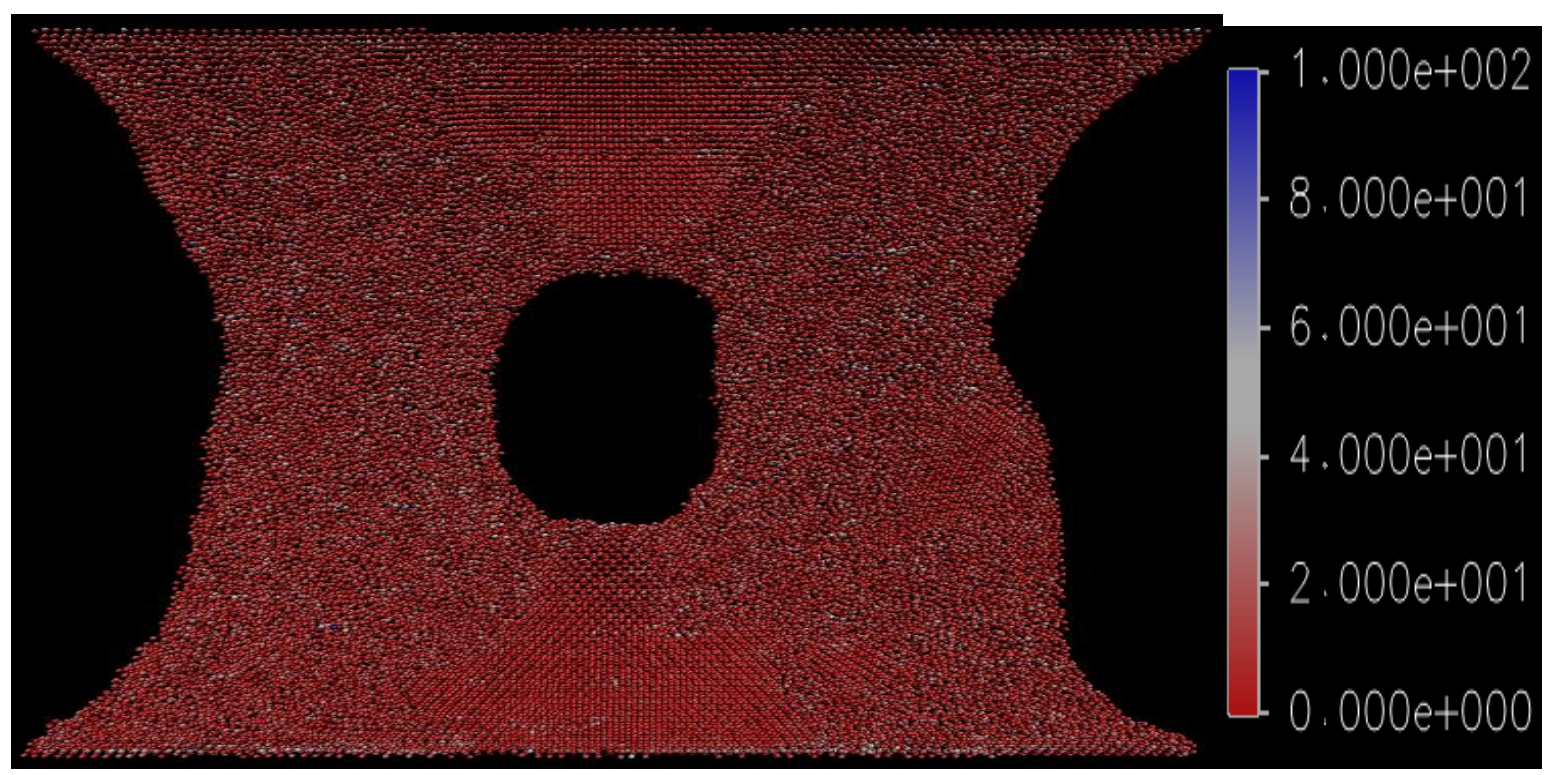

Figure 66: Increased crack blunting and crack elongation 


\subsubsection{At 800K}

At $800 \mathrm{~K}$ very high stresses are seen in zirconium with crack propagation and branching as seen in figure 67

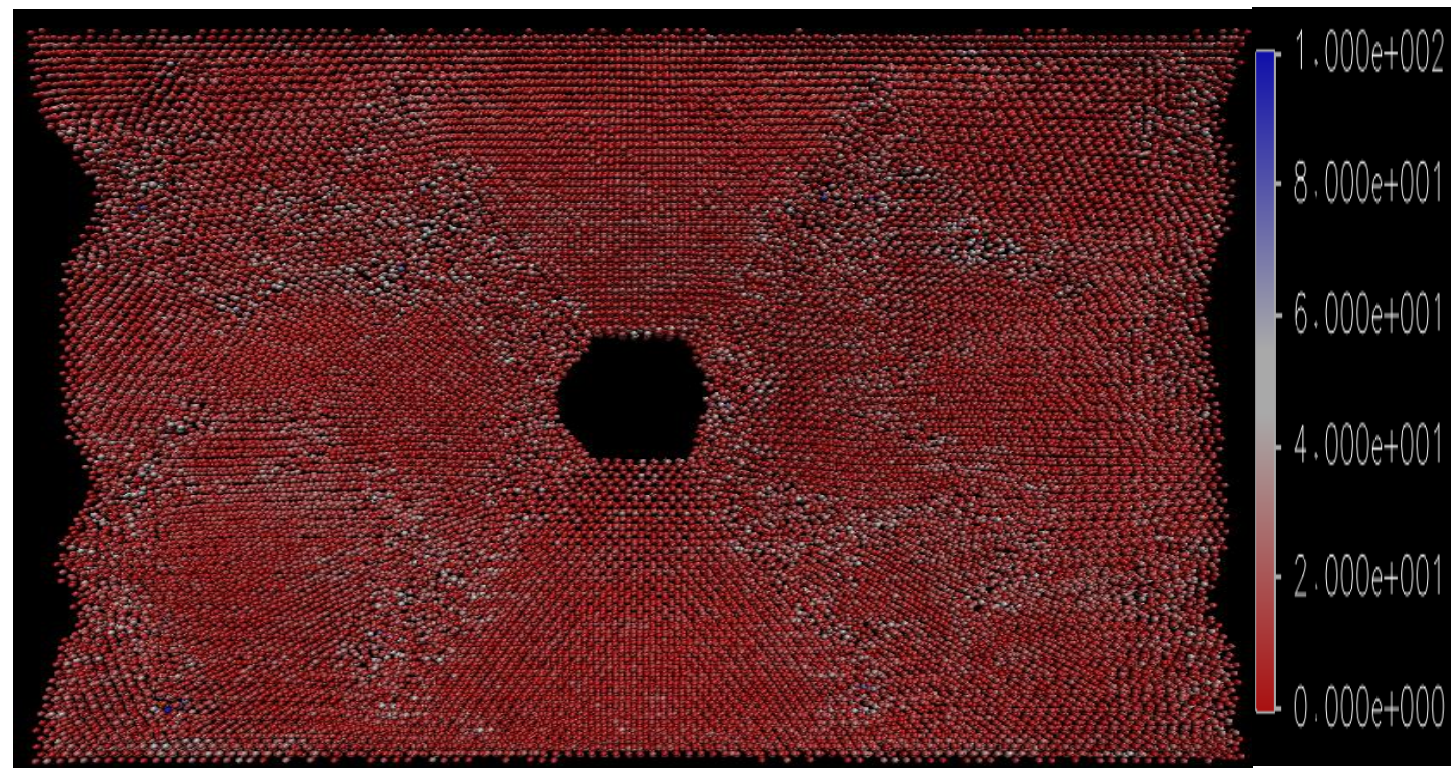

Figure 67: Crack branching on deformed zirconium due to high temperatures

\section{$\underline{\text { With hydrogen }}$}

In presence of hydrogen very high stresses are seen in zirconium with crack propagation and branching on highly deformed structure as seen in figure 68

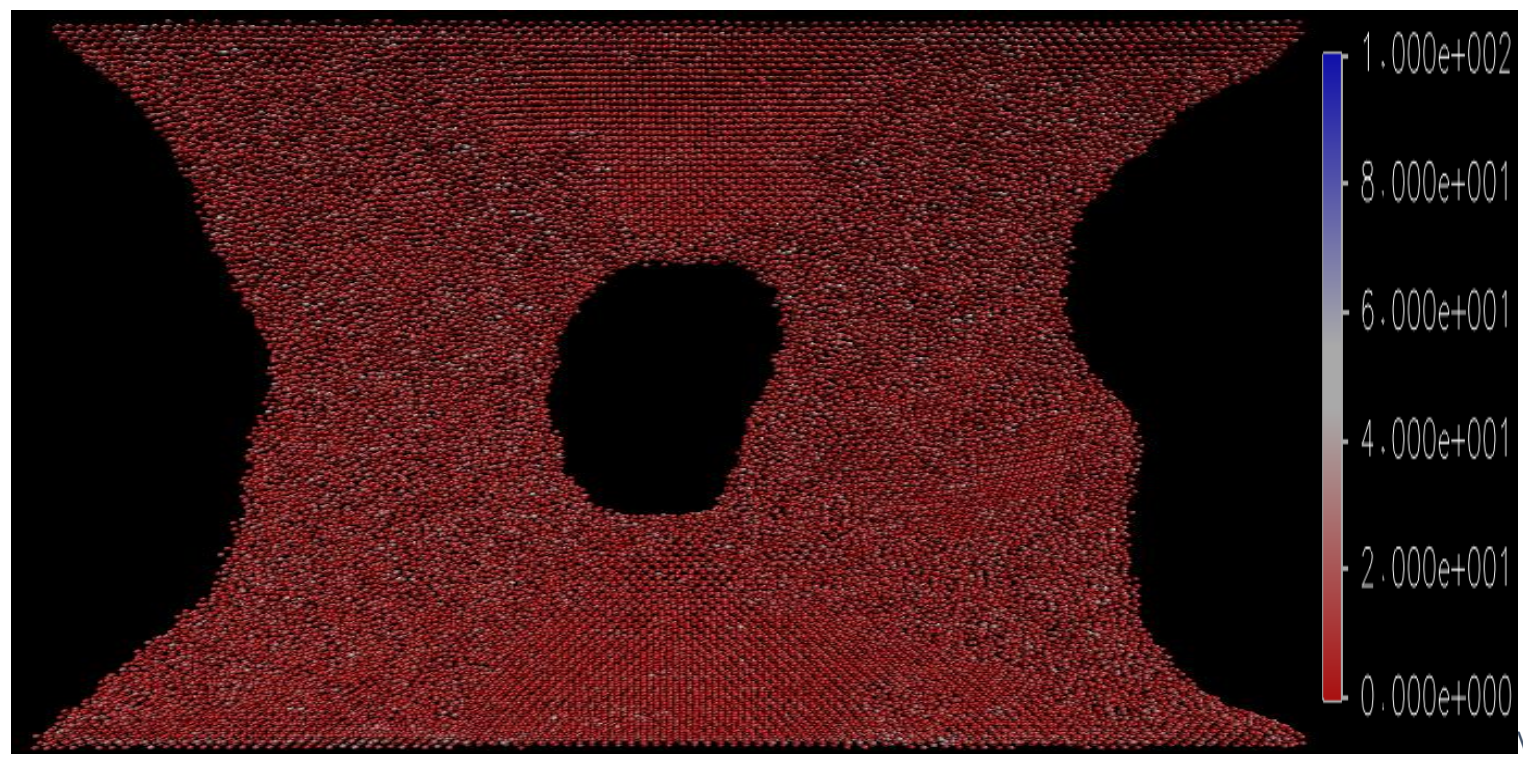

Figure 68: Enhanced crack blunting with high deformation in zirconium due to hydrogen 


\subsection{Strength of zirconium}

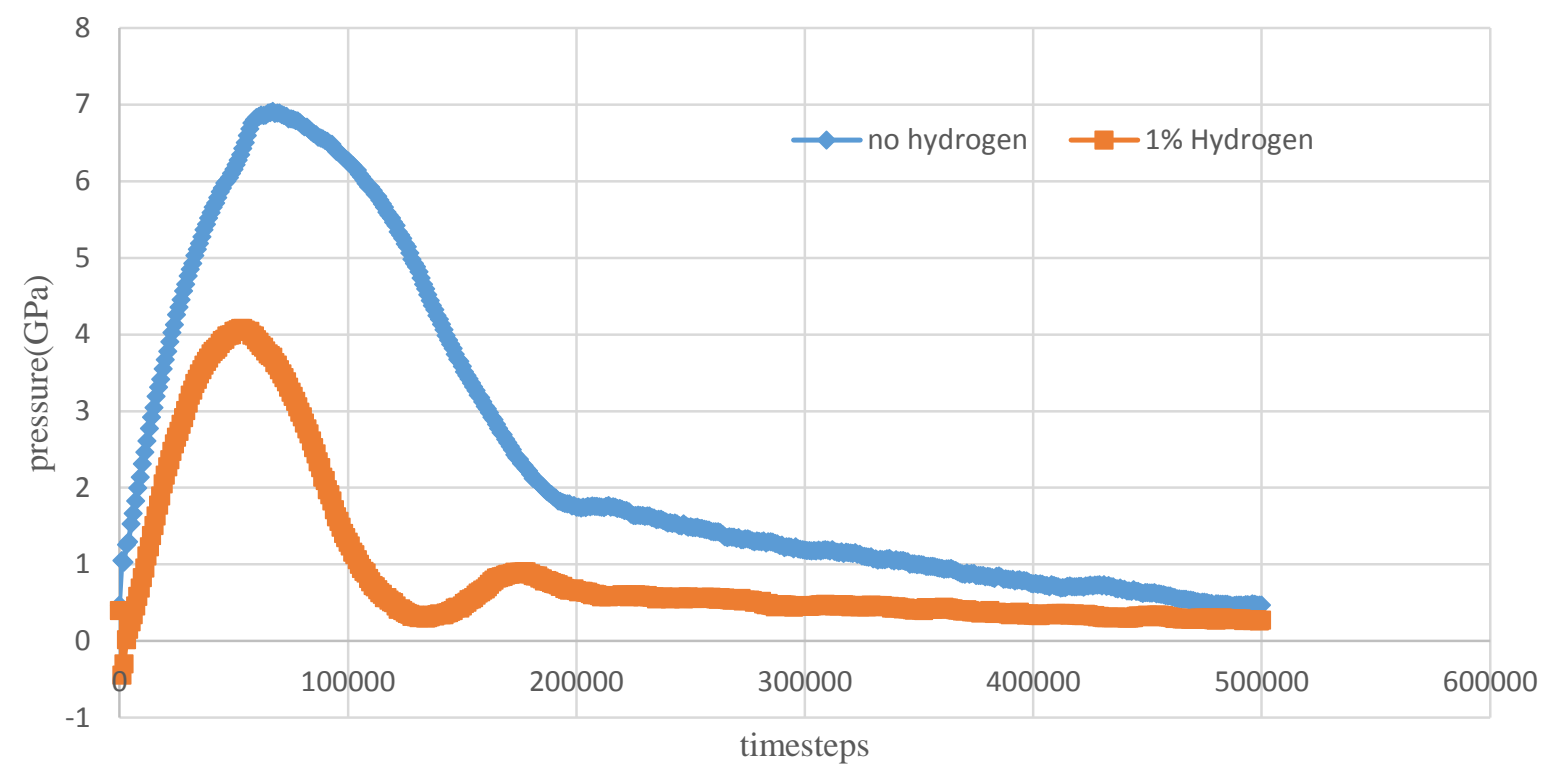

Figure 69: Indicates early crack nucleation in presence of hydrogen and loss of strength in zirconium

From the above figure 69 it clearly shows that hydrogen reduces the strength of material and nucleates the crack early upon applied external tensile strain. It also shows that the material deforms before reaching the yielding point.

9.8.1. Variation of crack propagation with increase in temperature in presence of hydrogen

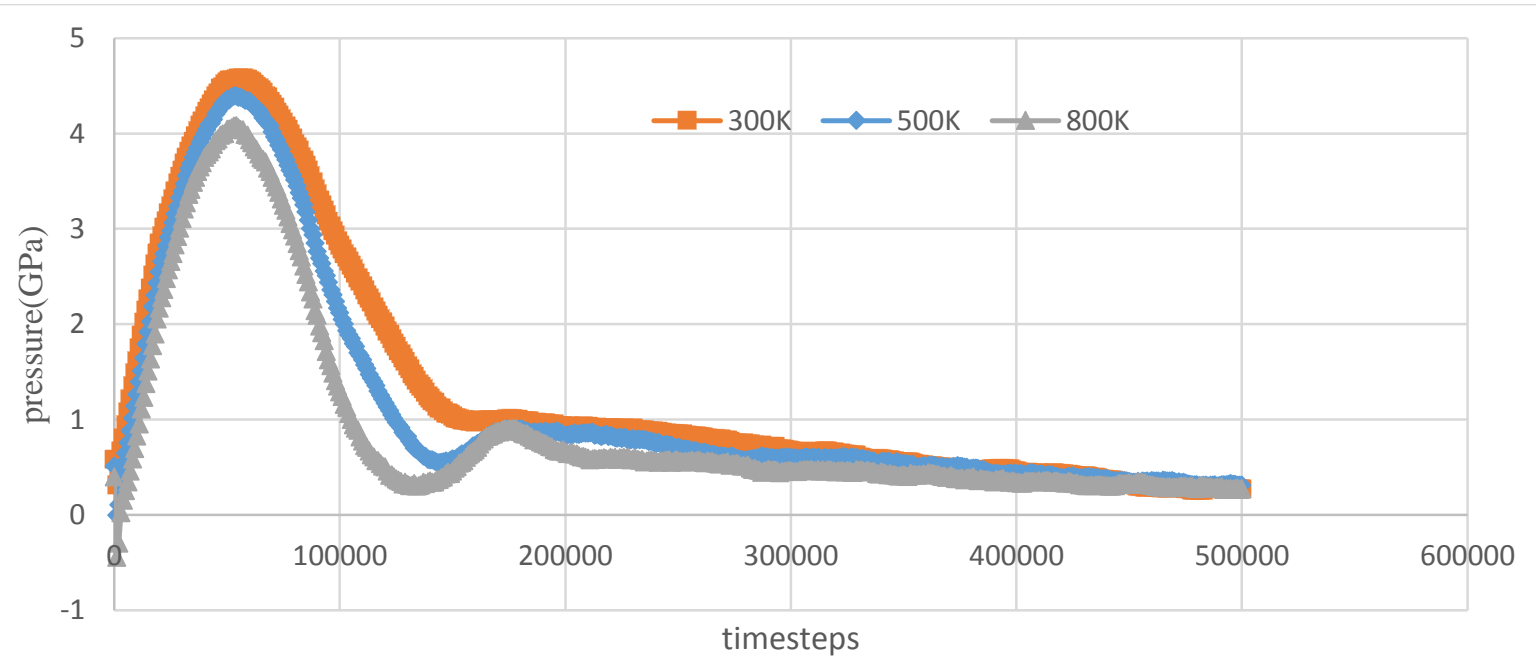

Figure 70: Stress reduction and crack nucleation with increase in temperature in presence of hydrogen 
The above figure 70 shows increase in temperature increases crack nucleation in presence of hydrogen thereby reducing the strength of zirconium.

\subsection{Conclusions}

- The bond angle analysis is done in OVITO software. From the bond angle analysis we can clearly see the effect of hydrogen in zirconium on crack propagation. The formation of weaker bonds between $\alpha-\mathrm{Zr}$ atoms is mainly due to presence of hydrogen and increase of strain.

- At $0 \mathrm{~K}$ when hydrogen is present the crack propagates easily and material fractures. Stress analysis is viewed VMD software indicate the presence of stress concentration around the crack as well as in places where hydrogen is present.

- The effect of hydrogen induced crack propagation and failure is validated through experimental observations.

- The effect of hydrogen on crack propagation is most clearly seen at lower temperatures but with the increase in temperature the stress is reduced and hydrogen contributes mainly to the crack elongation in the direction of applied strain.

- The hydrogen atom reduces the strength of material and at higher temperatures the crack most often elongates in the direction of the strain. The length of the crack along direction of the strain increase with increase of temperature.

- From the presented results we may conclude that in the presented model of crack propagation hydrogen reduces the strength of zirconium. 


\section{CHAPTER 10}

\section{SUMMARY OF RESULTS, LIMITATIONS AND FUTURE WORK}

\subsection{Summary of results}

- Minimized lattice parameters and cohesive energies for $\alpha-\mathrm{Zr}$ using MEAM and EAM potentials are found to be in good agreement with experimental data. Increase of lattice parameters with increase in temperature that is observed agree with the experimental data. Anisotropic thermal expansion is in good agreement with experiments. The stiffness values, Young's Modulus, Poisson's ratio and bulk Modulus for $\mathrm{Zr}$ are calculated using MEAM and EAM and agree well with experiments.

- Both EAM and MEAM potentials used in this research are validated for $\alpha-\mathrm{Zr}$ and further tested for presence of hydrides in order to decide the best potential for $\alpha-\mathrm{Zr}$ and zirconium hydride.

- Stability of zirconium hydride is tested using EAM and MEAM potentials. EAM potential is not suitable for the hydride structure. MEAM potential describes well phase transformation from face centered tetragonal to face centered cubic. Face centered cubic is found to be the stable structure for zirconium hydride. Good agreement of lattice parameters and stiffness constants for zirconium hydride with experimental data is observed.

- Mechanical properties of hydride are calculated and hydride has higher bulk modulus compared to zirconium which means that hydride is more brittle material. Increase in lattice parameters were observed with increase in temperature for hydride although there is no experimental data available for comparison.

- Increase in temperature causes lower stress and early yielding in zirconium. Tensile and compression stresses are reduced with increase in temperature Anisotropy of stress in zirconium is clearly observed using both EAM and MEAM potentials. Yielding is early in case of MEAM potential compared to EAM.

- Diffusion as a function of temperature is calculated using MEAM potential. Diffusion of hydrogen increases with increase in temperature from $500 \mathrm{~K}\left(1.92 * 10^{-7} \mathrm{~cm}^{2} / \mathrm{s}\right)$ to $1200 \mathrm{~K}$ $\left(1.47 * 10^{-4} \mathrm{~cm}^{2} / \mathrm{s}\right)$ are in good agreement with experimental data. Also, calculated 
activation energies are in good agreement with experimental data. Anisotropic hydrogen diffusion coefficients are obtained with increase in temperature and this coefficient is the

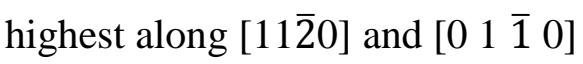

- Strain induced diffusion of hydrogen inside pure zirconium is calculated. Higher diffusion coefficient is obtained along [0001] direction of zirconium .Molecular dynamics give clear evidence that hydrogen diffusion is affected by strain. Effect of Diffusion is affected more by the compression strain and less by the tensile strain.

- Strain induced energy variation is studied on $\{111\}$ oriented hydride in order to provide possible explanation on hydride reorientation. Straining along $\left[\begin{array}{lll}1 & \overline{1} & 0\end{array}\right]$ direction the

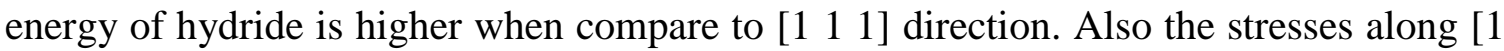
$\left.\begin{array}{ll}\overline{1} & 0\end{array}\right]$ is higher compared to [llll $\left.\begin{array}{ll}1 & 1\end{array}\right]$ direction. The high energy of the structure and stress along [1 $\left.\begin{array}{lll}1 & 0\end{array}\right]$ direction could possibly explain hydride reorientation on application of stress.

- Interface (0001) $\alpha-\mathrm{Zr} / /\{111\} \delta-\mathrm{ZrH}_{2}$ is modeled and energy minimized. The cohesive energy of the interface is calculated as $-4.547 \mathrm{eV} /$ atom. Increase stress show crack nucleation in hydride and this crack propagates into zirconium matrix. Increase of temperature shows early nucleation of crack causing fracture of zirconium tubes. Comparison with experimental observation validates the results of modelling of cracking.

- Effect of hydrogen on type of bonds formed (bond angle) in zirconium is calculated using OVITO software. The formation of weaker bonds at higher temperatures is also affected by presence of hydrogen. In the presence of hydrogen in zirconium the crack propagates easily and material fractures. This fracture due to hydrogen is in good agreement with experimental observation. With the increase of temperature from 300-800K the effect of hydrogen is observed as increase blunting of crack as compared to pure zirconium. Further stress analysis is done to understand the stresses inside zirconium in the presence of hydrogen using VMD software. From this analysis we see the presence of stress concentration around the crack as well as in places where hydrogen is present. Higher stress concentration is observed in the presence of hydrogen. 


\subsection{Limitation and Future Work}

The main limitation of molecular dynamic simulation is size of simulated structure. Larger structures requires more computation time and larger storage space. Therefore, calculations needs to be done on supercomputer's like one provided by Compute Canada.

This is the first test of LAMMPS code for EAM and MEAM for zirconium in presence of hydrogen (see Chapter 4). The extensive analysis of the results has been done and the detailed examples has been solved using the previously developed potentials for $\mathrm{Zr}-\mathrm{O}-\mathrm{H}$ systems (Chapter 4- 9). Through this research as described in Chapter 4, it has seen that EAM potential is validated for pure zirconium, however and in presence of hydrogen the potential does not give satisfactory results. MEAM potential can only be used for face centered cubic structure of zirconium hydride. Also MEAM for pure zirconium has weak interactions along one of the shear directions.

Future work of this research could be extended to studying diffusion of hydrogen with the presence of the defects like vacancies, grain boundaries and radiation defects. The MEAM potential which is used for zirconium and zirconium hydride can be extended to calculations of $\mathrm{Zr}$ alloys. Also future investigations can be done on polycrystalline structure with grain boundaries and different textures.

The methodology provided in the appendix can assist in future work on more complex system; it can also be adapted for materials used in new generation reactors with application of different alloys that sustain higher temperatures and supercritical water environment.. 


\section{REFERENCES}

1. Simpson, C.J., Ells, C.E. 1974, "Delayed Hydrogen embrittlement in Zr-2.5 wt\% Nb", Journal of Nuclear Materials, Vol (52), pp. 289-295.

2. Edsinger, K., Vaidayanathan, S., Adamson, R.B. 1999, "On the mechanism of axial splits in failed BWR fuel rods, Proceedings 9th international conference on environmental degradation of materials in nuclear power systems", The Metals and Materials Society, Warrendale, PA,pp.1201-1209.

3. Grigoriev, V., Josefsson, B. 1998, "On the mechanism of Zircaloy cladding axial splits", Journal of Nuclear Materials, Vol (257), pp.99-107.

4. Szpunar, J. A., Qin, W., Kiran Kumar, N.A.P., Li, Hualong. 2012, "Roles of texture in controlling oxidation, hydrogen ingress and hydride formation in $\mathrm{Zr}$ alloys", Journal of Nuclear Materials, Vol (427), pp.343-349.35

5. Hurst, D.G.1997," Canada Enters the Nuclear Age: A Technical History of Automic Energy of Canada Limited", McGill-Queen's University Press, Quebec

6. Webster, R.T.1974,"Zirconium for nuclear primary steam systems", American society for testing and materials, Portland,USA, pp.5-13.

7. C., Goren, S. D. 1986, "NMR study of hydrogen diffusion in zirconium hydride", Physical Review B, Vol(33), pp.68-78.

8. Kiran Kumar, N.A.P., Szpunar, J. A., He, Zhang. 2010, "Preferential precipitation of hydrides in textured zircaloy-4 sheets", Journal of Nuclear Materials, Vol (59), pp. 7010-7021. 
9. Qin, W., Kiran Kumar, N.A.P., Szpunar, J. A., Kozinski,J.2011, “Intergranular $\delta$ hydride nucleation and orientation in zirconium alloys", Journal of Nuclear Materials, Vol (403), pp. 101-107.

10. Shmakov, A. A., Yan, D., Eadie, R. L. 2006, “A Theoretical and experimental study of hydrides in zirconium Alloys”, Metal Science and Heat Treatment, Vol(48), pp. 146-149.

11. Mendelev, M. I., and Ackland, G. J. 2007, "Development of an interatomic potential for the simulation of phase transformations in zirconium", Philosophical Magazine Letters, Vol (87), pp. 349-359.

12. Szpunar, B., Baskes, M.I., Jochym, P.T. 2003, "Oxygen and Hydrogen diffusion in Zirconia”, 8th International Conference on CANDU fuel, Honey Harbour, Canada, pp.551-557.

13. Bowman Jr, R.C., Venturini E.L., Craft B.D., Attalla A., Sullenger D.B. 1983, "Electronic structure of zirconium hydride: A proton NMR study", Physical Review B, Vol(27), pp.1474-488.

14. Peng Zhang., Bao-Tian Wang., Chao-Hui Hea., Ping Zhang.2011, "First-principles study of ground state properties of $\mathrm{ZrH}_{2}$ ", Computational Materials Science, Vol (50), pp. 3297-3302.

15. Daw, S. M., Baskes, M. I.1983, “Semiempirical, Quantum Mechanical Calculation of Hydrogen Embrittlement in Metals", Physical Review Letters, Vol (50), pp. 12851288.

16. Baskes, M. I. 1992, “Modified embedded-atom potentials for cubic materials and impurities”, Physical Review B, Vol (46), pp.2727-2742. 
17. Goldak, J., Lloyd, L. T., Barrett, C. S.1966, "Lattice Parameters, Thermal Expansions, and Grüneisen Coefficients of Zirconium, 4.2 to $1130^{\circ} \mathrm{K}$ ”, Physical Review, Vol (144), pp.478-483.

18. Skinnert, G. b. , Johnston,H.L.1952, "Thermal Expansion of Zirconium between $298^{\circ} \mathrm{K}$ and $1600^{\circ} \mathrm{K}$ ", The Journal Of Chemical Physics, Vol (21),pp.1383-1384.

19. Wipf , H. , Kappesser, B., Werner R. 2000, "Hydrogen diffusion in titanium and zirconium hydrides", Journal of Alloys and Compounds, Vol (310), pp. 190-195

20. 28-58 Jr, H.L. 1958," Thermocrystallography of higher hydrides of titanium and zirconium", Acta Crystallography, Vol (11), pp. 46-51.

21. Neogy, S., Srivastava, D., Tewari, R., Singh, R.N., Dey, G.K., Banerjee, S. 2003,” Microstructural study of hydride formation in $\mathrm{Zr}-1 \mathrm{Nb}$ alloy", Journal of nuclear materials, Vol (322), pp.195-203

22. Mayilyan, D.G. 2007,'Investigation of alloys formation from binary hydrides in ZrHf system,’ICHMS, Sudak, Ukraine, pp.272-273.

23. Domain, C., Besson, R., Legris, A. 2003, "Atomic-scale ab initio study of the $\mathrm{Zr}-\mathrm{H}$ system: II. Interaction of $\mathrm{H}$ with plane defects and mechanical properties", Acta Materialia, Vol (52), pp.1495-1502.

24. Switendick, A.C. 1984, "Electronic structure of $\gamma$ phase zirconium hydride", Journal of the Less Common Metals, Vol (103), pp.309-315.

25. Paolucci,G., Colavita,E., Weaver,J.H.1985, "Thermoreflectance investigation of zirconium hydrides in the face-centered-tetragonal phase", Physical Review B,Vol (32), pp.2610-2613. 
26. Lejcek, P.2010,"Grain boundary segregation in metals", Springer Series Materials, Vol (136), pp 5-24.

27. Une, K., Nogita, K., Ishimoto, S., Ogata,K. 2004, "Crystallography of Zirconium Hydrides in Recrystallized Zircaloy-2 Fuel Cladding by Electron Backscatter Diffraction", Journal of nuclear science and technology, Vol (41), pp. 731-740

28. Colas, K.B, Motta, A.T., Almer, J.D., Daymond, M.R., Kerr, M., Banchik, A.D., Vizcaino , P., Santisteban, J.R.2010, "In situ study of hydride precipitation kinetics and re-orientation in Zircaloy using synchrotron radiation", Acta Materialia,Vol (58), pp.6575-6583.

29. Hong S.I., Lee. K.W. 2007, "Hydride reorientation in Zircaloy-4 cladding", Journal of Nuclear Materials, Vol ( 373), pp. 319-327

30. Chu, H.C., Wu, S.K., Chien, K.F., Kuo, R.C.2007, "Effect of radial hydrides on the axial and hoop mechanical properties of Zircaloy-4 cladding", Journal of Nuclear Materials, Vol (362), pp. 93-103

31. Ercolessi, F.1997, "A molecular dynamics primer", Spring College in Computational Physics, ICTP, Trieste

32. Allen, M. P.2004, "Introduction to Molecular Dynamics Simulation", Computational Soft Matter: From Synthetic Polymers to Proteins, Lecture Notes", Vol (23), pp.1-28.

33. Narayan,K., Behdinan,K., Fawaz, Z.2006, “An engineering-oriented embedded-atommethod potential fitting procedure for pure fcc and bcc metals", Journal of Materials Processing Technology, Vol (182), pp. 387-397

34. Daw, S. M., Foiles, S. M., Baskes, M. I. 1993, "The embedded-atom method: a review of theory and applications", Materials Science Reports, Vol (9), pp. 251-310. 
35. Baskes M. I., Johnson R. A.1994, "Modified embedded atom potentials for HCP metal", Modelling Simulation of Material Science Engineering, Vol (2), pp.147-163.

36. Plimpton, S. 1995, "Fast Parallel Algorithms for Short-Range Molecular Dynamics", Journal of Computational Physics, Vol (117), pp.1-19.

37. Stukowski, A. 2010, "Visualization and analysis of atomistic simulation data with OVITO - the Open Visualization Tool”, Modelling and Simulation Material Science and Engineering, Vol (18), pp. 1-7.

38. Humphrey, W., Dalke, A. and Schulten, K.1996, "VMD - Visual Molecular Dynamics", Journal of Molecular Graphics, Vol (14), pp. 33-38.

39. Lu, Jian. 1995, "Computer Modelling of Intergranular Fracture in textured materials" Phd thesis, McGill University, Montreal.

40. Pearson W.B. 1967, A Handbook of Lattice Spacings and Structures of Metals, Pergamon Press, Oxford.

41. Barrett C. S., Massalski T. B. 1966, Structure of Metals, McGraw-Hill, New York.

42. Simmons, G., Wang, H. 1971, Single Crystal Elastic Constants: A Handbook, MIT Press, Cambridge.

43. Tromans, D.2011, "Elastic Anisotropy of HCP Metal Crystals and Polycrystals", Vol (6), IJRRAS, pp.462-483.

44. Mayilyan, D.G. 2007,'Investigation of alloys formation from binary hydrides in ZrHf system,'ICHMS”, Sudak, Ukraine, pp.272-273. 
45. Bushow, K.H.J, Bouten, P.C.P and Meidema, A.R. 1982, "Hydrides formed from Intermetallic compounds of two transition metals: a special class of Ternary Alloys", Report on progressive physics, Vol(45), ppt 937- 1039.

46. Brandes, E.A. 1983, “Smithells Metals Reference books”, Buttlerworths, London.

47. David,W.O., Gillis,H.P., and Nachtrieb,N.H. 1999, "Principles of Modern Chemistry", Harcourt College Publisher ,Texas.

48. Kim, Young-Min ., Lee, Byeong-Joo., and Baskes, M. I.2006, "Modified embeddedatom method interatomic potentials for Ti and Zr", PHYSICAL REVIEW B, Vol (74) , pp. 014101-12

49. Kearns,J.J. 1972, "Diffusion of hydrogen in alpha zirconium, Ziracloy-2, and Zircaloy -4", Journal of Nuclear Materials, Vol 43, Issue 3, pp.330-338

50. Einstein, A. 1905, "Ü ber die von der molekularkinetischen Theorie der Wa“rme geforderte Bewegung von in ruhenden Flu“ssigkeiten suspendierten Teilchen”, annalen der physic, Vol (17), pp. 549-560.

51. Shackelford, J. F., "Introduction to materials science for engineers", Prentice Hall, 2009.

52. Kiran Kumar, N.A.P, Ph.D. thesis, McGill University; 2011.

53. Zanellato, O., Preuss , M. , Buffiere, J.Y., Ribeiro, F. , Steuwer , A. , Desquines, J., Andrieux, J. , Krebs, B., "Synchrotron diffraction study of dissolution and precipitation kinetics of hydrides in Zircaloy-4 
54. Kotrechko,S .A., Filatov, A. V., Ovsjannikov,A. V. 2006, "Molecular dynamics simulation of deformation and failure of nanocrystals of bcc metals". Theoretical and Applied Fracture Mechanics, Vol (45), pp. 92-99.

55. Huang, Dan., TAO, Wei-ming., GUO, Yi-mu.2005, "Molecular dynamics simulation of failure process of nano aluminum wire under axial tension". Ordnance Material Science and Engineering, Vol (28), pp.1-4

56. Kitamura, T., Hirakate, H., Satake,Y. 2004, "Applicability of fracture mechanics on brittle delamination of nanoscale film edge", JSME International Journal (Series A), Vol (47), pp. 106-112.

57. Ikeda, T., Miyazaki, N. 1998, "Mixed mode fracture criterion of interface crack between dissimilar material", Engineering Fracture Mechanics, Vol (59), pp. $725-735$.

58. Ackland, G. J., Jones, A. P.2006, "Applications of local crystal structure measures in experiment and simulation", Physical Review B, Vol (73), pp. 0541041- 0541047.

59. Bertolino, G ., Meyer,G., Perez Ipin̂a,J.2003, "In situ crack growth observation and fracture toughness measurement of hydrogen charged Zircaloy-4", Journal of Nuclear Materials , Vol 322, pp. 57-65 


\begin{abstract}
APPENDIX
The following folders contain record of Molecular dynamics calculations performed using LAMMPS code on Bugaboo and Zeno (Westgrid) computers. The following files and software are placed in bugaboo and on PC with description for future reference.

\section{Lattice Parameters and cohesive energy folder contains calculations for EAM and MEAM for zirconium as discussed in Chapter 4}

\title{
Methodology:
}

Lattice parameters for zirconium are tested using various lengths in lattice command shown in below code for both eam and meam potentials. Periodic boundary conditions with metal units and atomic style is applied in order to study zirconium. Potential energy of each atom is computed and is used for calculation of minimum lattice parameters. Timestep used is 0.001 psec. Energy and pressure are specified using fix command with necessary thermo data is printed. When the lowest energy is reached the program outputs the lattice parameters ' $a$ ' and 'c'. The output of the program is dumped in 'lattice.out' file.A similar approach is applied for zirconium hydride with change in lattice command and pairstyle specifying hydrogen as shown in code.The code is a modification of Mark Tschopp found in https://icme.hpc.msstate.edu/mediawiki/index.php/LAMMPS Tutorial 1

\# Sample program to calculate lattice parameters for pure $\alpha$-Zr using EAM and MEAM

$\log$ lattice.out

units metal

boundary $\mathrm{p} \mathrm{p} \mathrm{p}$

atom_style atomic

pair_style meam \# For MEAM potential

\#pair_style eam for EAM potential

lattice hcp 3.232

\#lattice fcc 4.83 for zirconium hydride

region box block 050303

create_box 1 box

create_atoms 1 box

mass $\quad 191.224$

\#mass 21.0079 (in case of hydrogen)

pair_coeff $* *$ meamf $\mathrm{ZrN} \mathrm{ZrH} 2$. meam ZrN(For MEAM)

\#pair_coeff $* *$ Zrncs.eam for Zr EAM potential

\# pair_coeff $\quad * *$ meamf $\mathrm{ZrN} \mathrm{Hz} \mathrm{ZrH2.meam} \mathrm{ZrN} \mathrm{Hz} \mathrm{(For} \mathrm{MEAM} \mathrm{with} \mathrm{hydrogen)}$

\# regions of sample

neighbor 2.0 bin

neigh_modify delay 0 every 1

dump 1 all custom 100 atom.dump id type $\mathrm{x} \mathrm{y} \mathrm{z}$

dump_modify 1 every 100

\# --------- Define Settings

compute eng all pe/atom

compute eatoms all reduce sum c_eng 


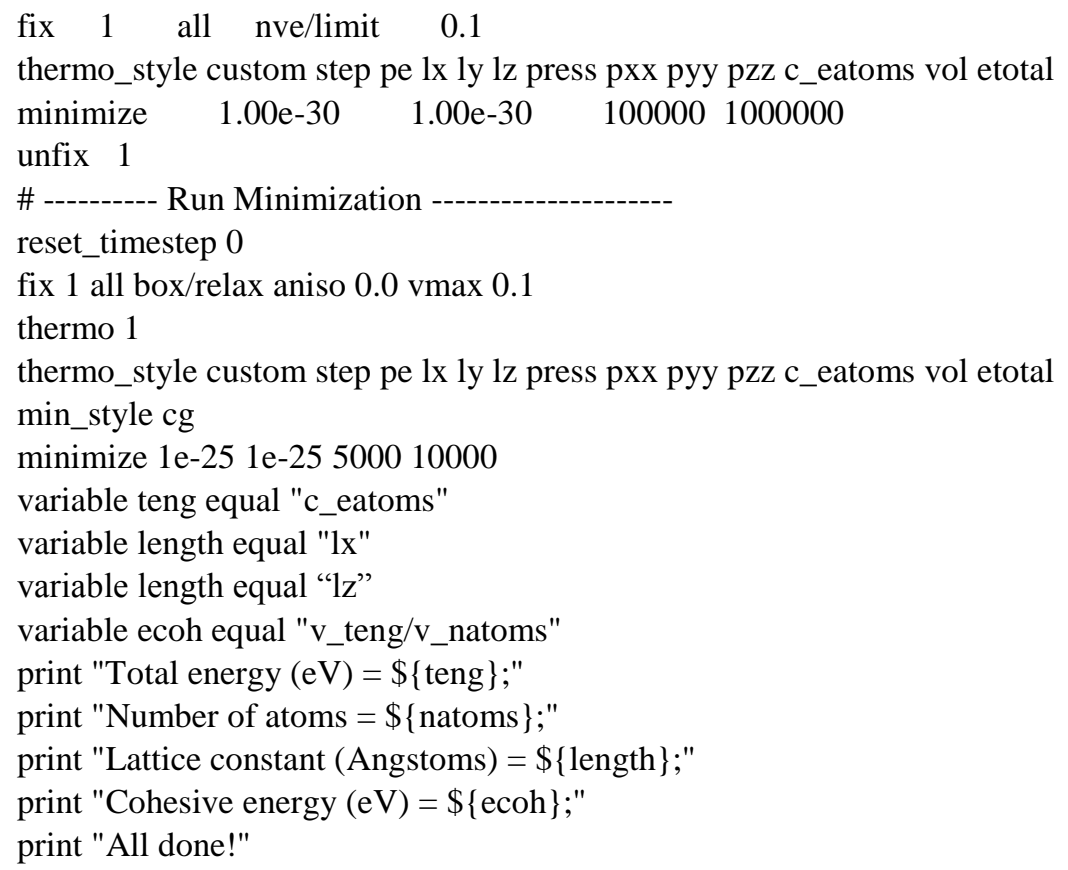

Script to run the above program in Westgrid

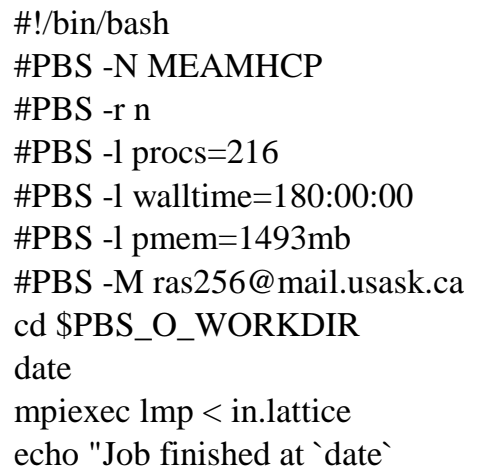

\section{Variation of lattice parameters with temperature as discussed in Chapter 4}

\section{Methodology:}

Increase of lattice parameters is verified for zirconium and zirconium hydride by using minimum energy structure. The pressure along each direction is specified as 0 bars and temperature used is $500 \mathrm{~K}$ in below script. Equilibration times vary for different temperatures. In case of presence of hydrogen the timestep is chosen as $0.0001 \mathrm{psec}$. Initial velocity is chosen similar to temperature required and any angular momentum is removed. Thermo outputs temperature, pressure, volume, these values are used to check for convergence and lx, ly and lz box lengths are used to calculate lattice parameters. 
Sample script to calculate lattice parameters at various temperatures

log lattice. Out

units metal

boundary $\mathrm{p} \mathrm{p} \mathrm{p}$

atom_style atomic

pair_style meam

lattice hcp 3.232

region box block 016010010

create_box 1 box

create_atoms 1 box

mass $\quad 191.224$

pair_coeff $* *$ meamf $\mathrm{ZrN} \mathrm{ZrH} 2$. meam $\mathrm{ZrN}$

\# regions of sample

neighbor 2.0 bin

neigh_modify delay 0 every 1

reset_timestep 0

velocity all create 500. 12345 mom yes rot no

fix 1 all npt temp 500. 500. 1 aniso 001 drag 0.5

compute 2 all temp

dump $\quad 1$ all custom 1000 temp3.dump id type $\mathrm{xu} \quad \mathrm{yu} \quad \mathrm{zu} \quad$ ix $\quad$ iy iz

dump_modify 1 every 10000

\# Set thermo output

thermo 10000

thermo_style custom step temp pe lx ly lz press pxx pyy pzz c_eatoms vol etotal

run 1000000

unfix 1

\section{Stiffness parameters as discussed in Chapter 4}

\section{Methodology}

To calculate stiffness for structure, Adian Thompson of Sandia Labs developed a script which uses 3 files in order init.mod, potential.mod and displace.mod .The sample script can be found in LAMMPS software.

Init.mod - requires crystal structures, units, deformation parameters and initial configuration of the atoms and simulation cell. The details of script are given in LAMMPS software.

potential.mod - pair styles and pair coefficients

displace.mod - Perform positive and negative box displacements

Different deformation rates are varied to test structure stability and final output is calculates stiffness values in 6 different directions.

\section{Stress and strain as discussed in Chapter 5}

\section{Methodology}

Stress at constant strain rate is calculated for zirconium strain diffusion, strain energy and Interface crack in the above described chapters. The stresses are calculated for different temperatures. The equilibrated structure obtained from minimum lattice parameters is used to do stress analysis. The following script calculates stresses at various temperatures. The structure is equilibrated to desired temperature, pressure and then constant strain is applied. Different strains can be varied in different directions of crystal. 


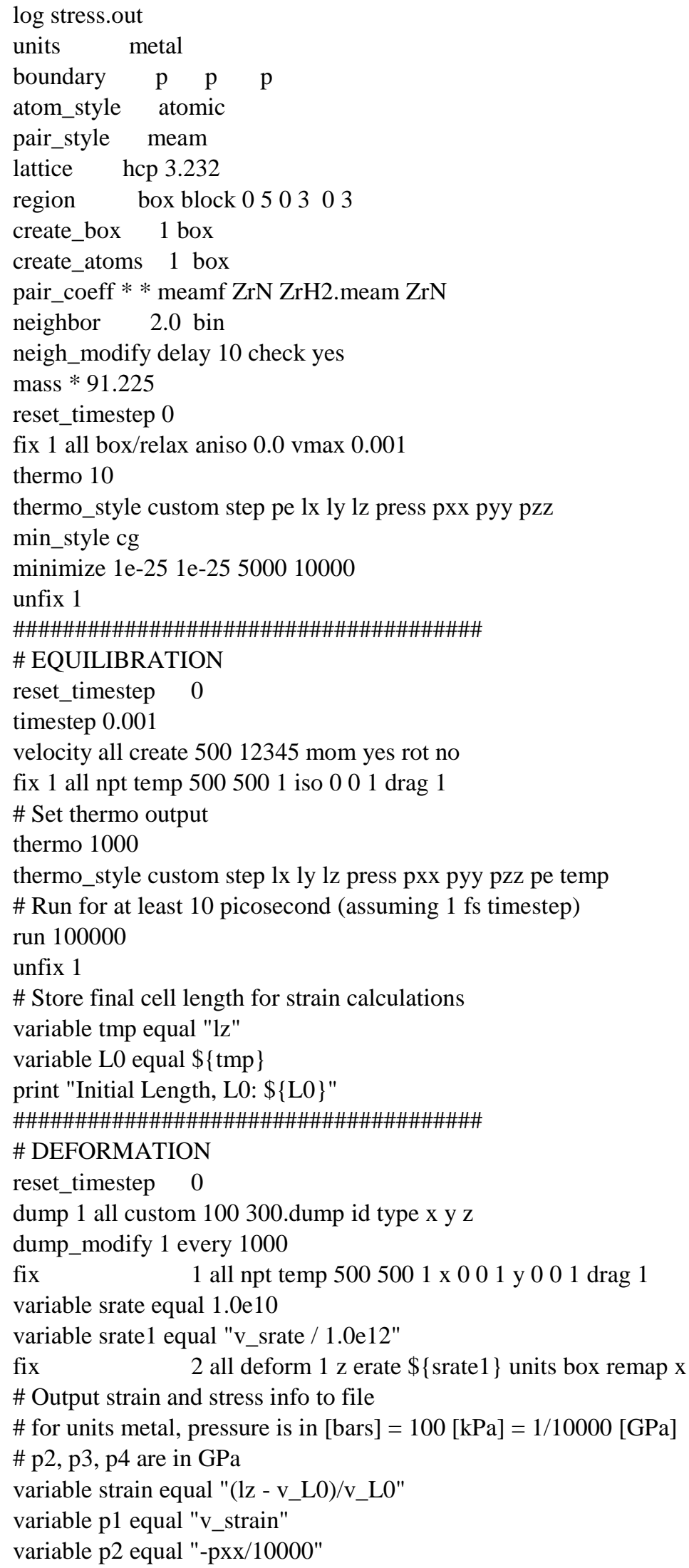


variable p3 equal "-pyy/10000"

variable p4 equal "-pzz/10000"

\# Display thermo

thermo 1000

thermo_style custom step v_strain temp v_p2 v_p3 v_p4 ke pe press

run 30000

\# SIMULATION DONE

print "All done"

\section{Diffusion of hydrogen in zirconium as discussed in Chapter 6}

\section{Methodology}

Complex problems like diffusion of hydrogen in zirconium is calculated for various temperatures and coefficients are calculated using Einstein's diffusion equation. The method used here is hydrogen atoms are randomly distributed and energy immixed . The script equilibrates and calculates the mean square displacement at desired temperatures. Timestep of 0.0001 psec is used. MSD without drifts (drifts in center of mass) are removed and displacement along 3 directions are dumped in file 500.txt.Maximum runs are done for 20000000 psec to test the variation in slopes. Average RDF calculations are done on zirconium to test the structure.

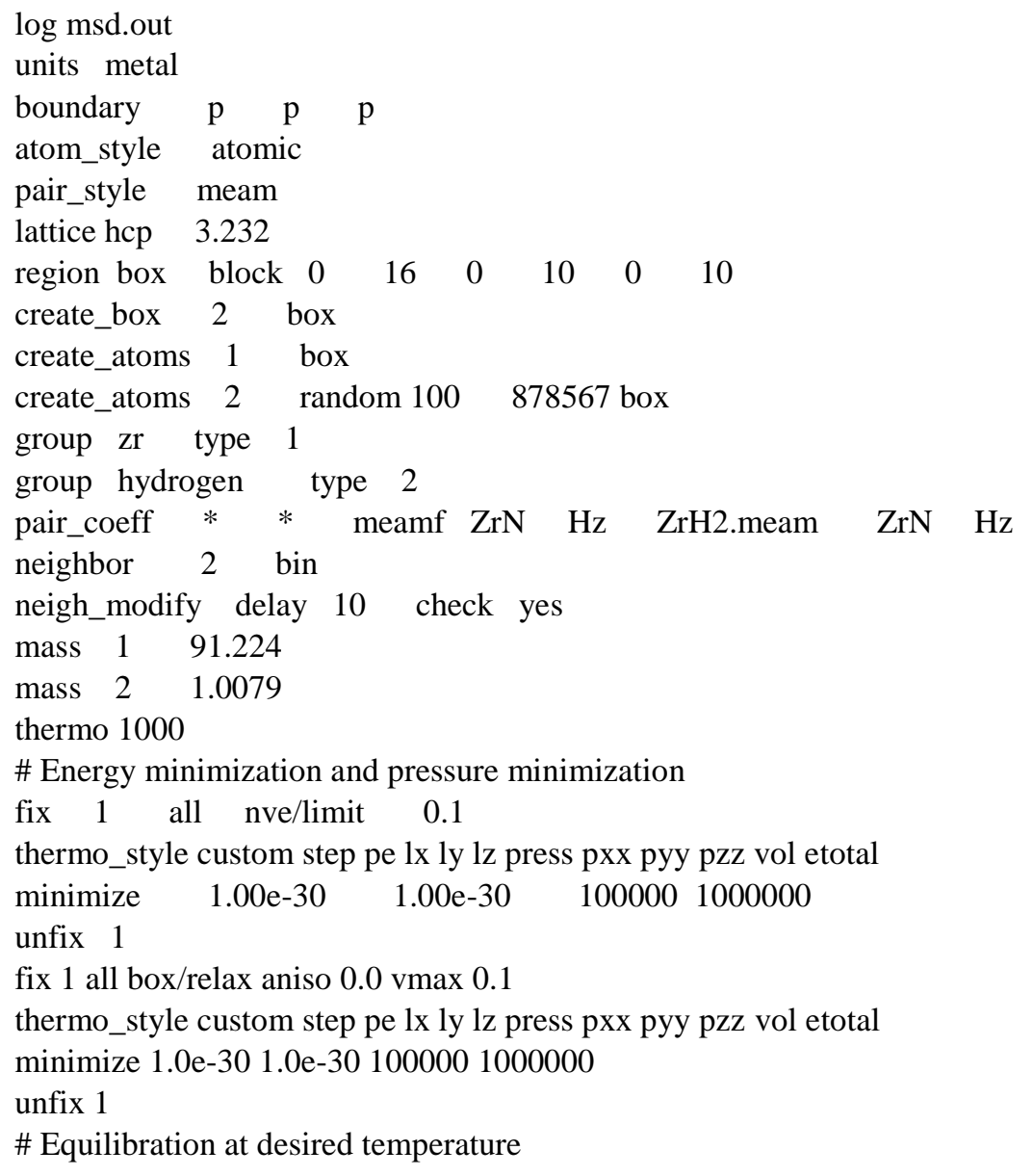




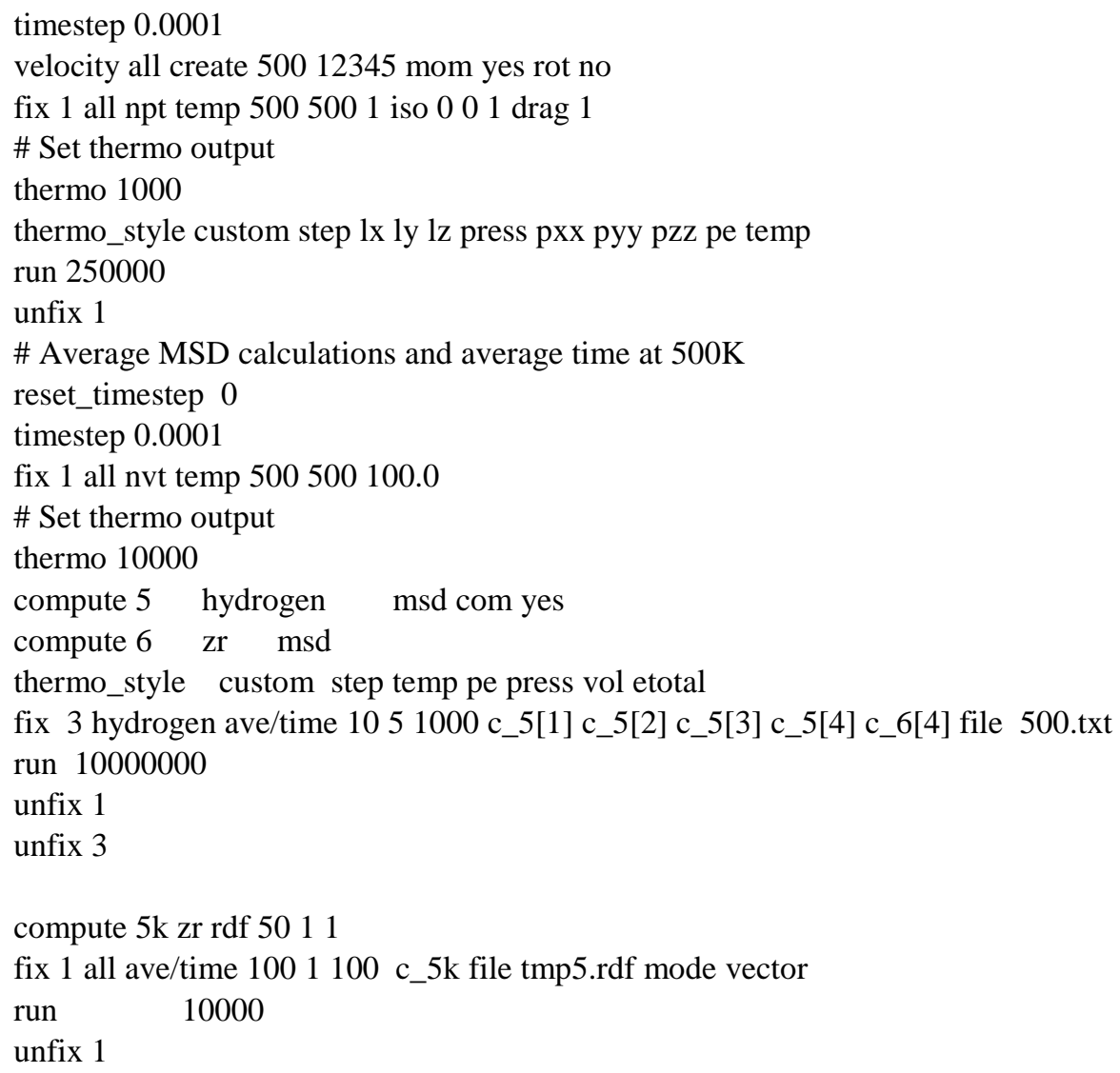

\section{Strain diffusion calculations as discussed in Chapter 6}

\section{Methodology}

Strain induced diffusion is done on zirconium with hydrogen placed inside a sphere of zirconium.The structure is energy and pressure minimized. It is then equilibrated and MSD is calculated before strain. The structure is then deformed and MSD along 3 directions is calculated using the following code.

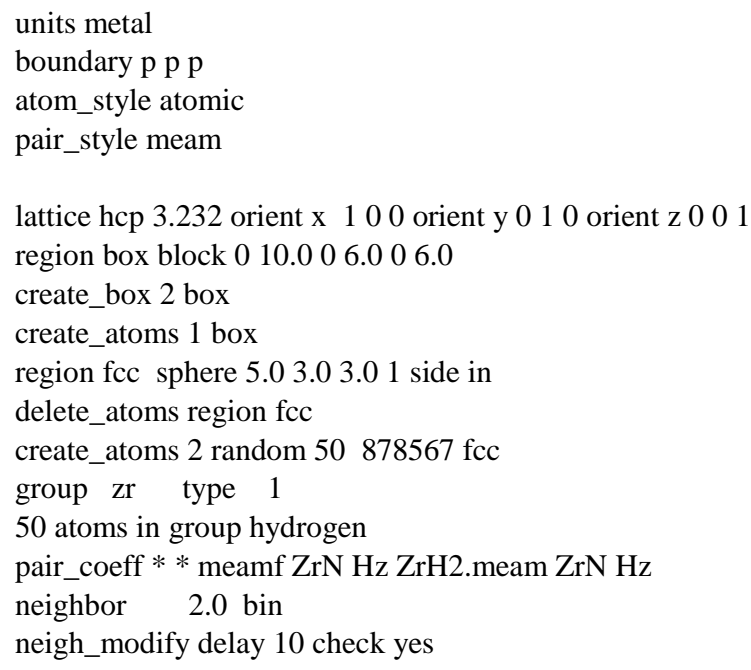




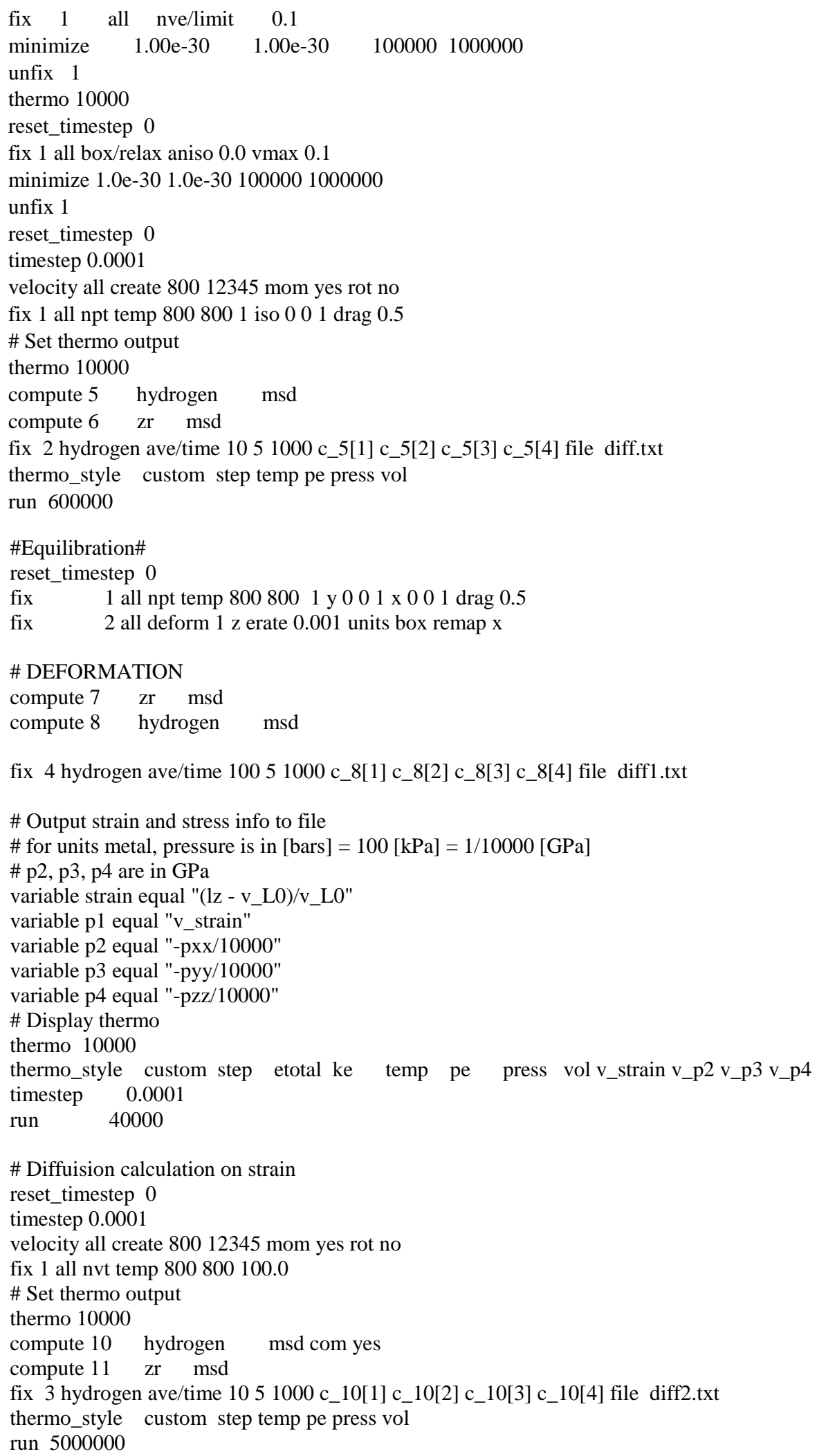




\section{Energy111 folder contains calculations for reorientation energy as discussed in Chapter 7}

\section{Methodology}

Hydride is oriented along [111] and minimized structure is calculated. The structure is then equilibrated at required temperature and different strain rates are tested for stable structure.

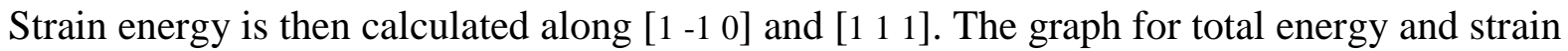
rate with corresponding stresses is plotted. The energy of two directions is compared for lowest energy orientation.

$\log$ xenergy.out

units metal

boundary $\mathrm{p} \mathrm{p} \mathrm{p}$

atom_style atomic

pair_style meam

lattice fcc 4.83 orient x $1-10$ orient y $111-2$ orient $\mathrm{z} 111$

region box block 08.007 .006 .0

create_box 2 box

create_atoms 1 box

lattice sc 2.415 origin 0.50 .50 .5 orient x $1-10$ orient y $11-2$ orient z 111

create_atoms 2 box

group zr type 1

group hydrogen type 2

pair_coeff $* *$ meamf $\mathrm{ZrN} \mathrm{Hz} \mathrm{ZrH2.meam} \mathrm{ZrN} \mathrm{Hz}$

neighbor 2.0 bin

neigh_modify delay 10 check yes

fix 1 all nve/limit 0.1

thermo_style custom step lx ly lz press pxx pyy pzz pe temp

minimize $\quad 1.00 \mathrm{e}-30 \quad 1.00 \mathrm{e}-30 \quad 1000001000000$

unfix 1

reset_timestep 0

fix 1 all box/relax aniso 0.0 vmax 0.1

thermo_style custom step lx ly lz press pxx pyy pzz pe temp

minimize 1.0e-30 1.0e-30 1000001000000

unfix 1

timestep 0.0001

velocity all create 623.12345 mom yes rot no

compute 1 hydrogen msd com yes

compute 2 zr msd

fix 1 all npt temp 623. 623. 1 iso 001 drag 0.5

thermo 10000

thermo_style custom step temp pe press vol etotal

run 1000000

unfix 1

thermo_style custom step lx ly lz press pxx pyy pzz pe temp

run 1

\# Store final cell length for strain calculations

variable tmp equal "lz"

variable L0 equal $\$\{$ tmp $\}$

print "Initial Length, L0: $\$\{\mathrm{~L} 0\}$ "

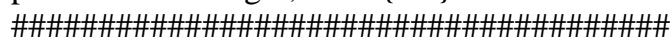




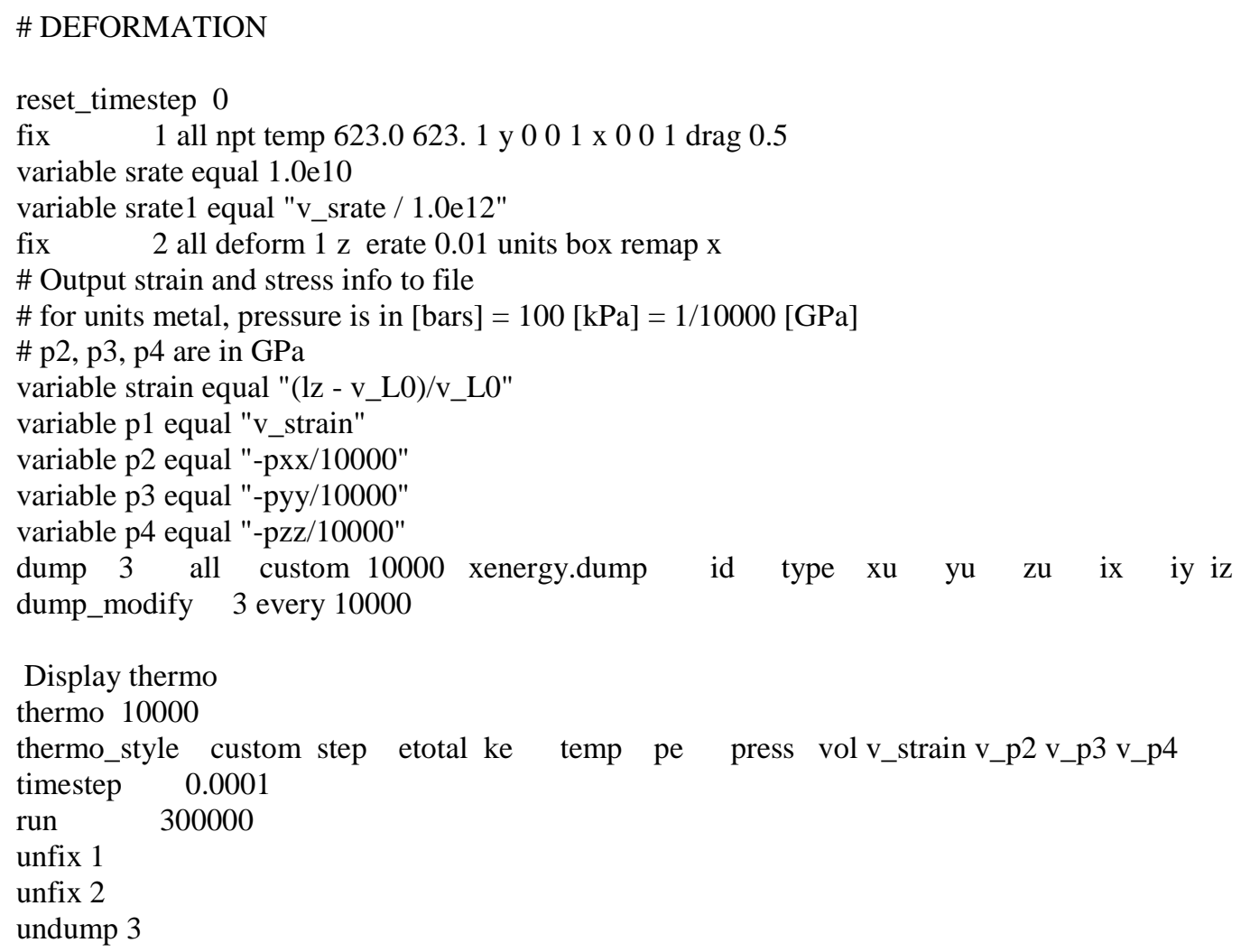

\section{Crack Interface folder contains calculations for MEAM as discussed in Chapter 8}

\section{Methodology}

Interface $(0001) \alpha-\mathrm{Zr} / /\{111\} \delta-\mathrm{ZrH}_{1.5}$ is created using FORTRAN programming language. The distance at the interface is similar to the distance of zirconium along 'c' directions. Interface is tested for stability and minimized energy. The following script calculates lattice parameters and cohesive energies. The structure is then equilibrated at various temperatures and a constant strain rate is applied on Interface to study crack initiation

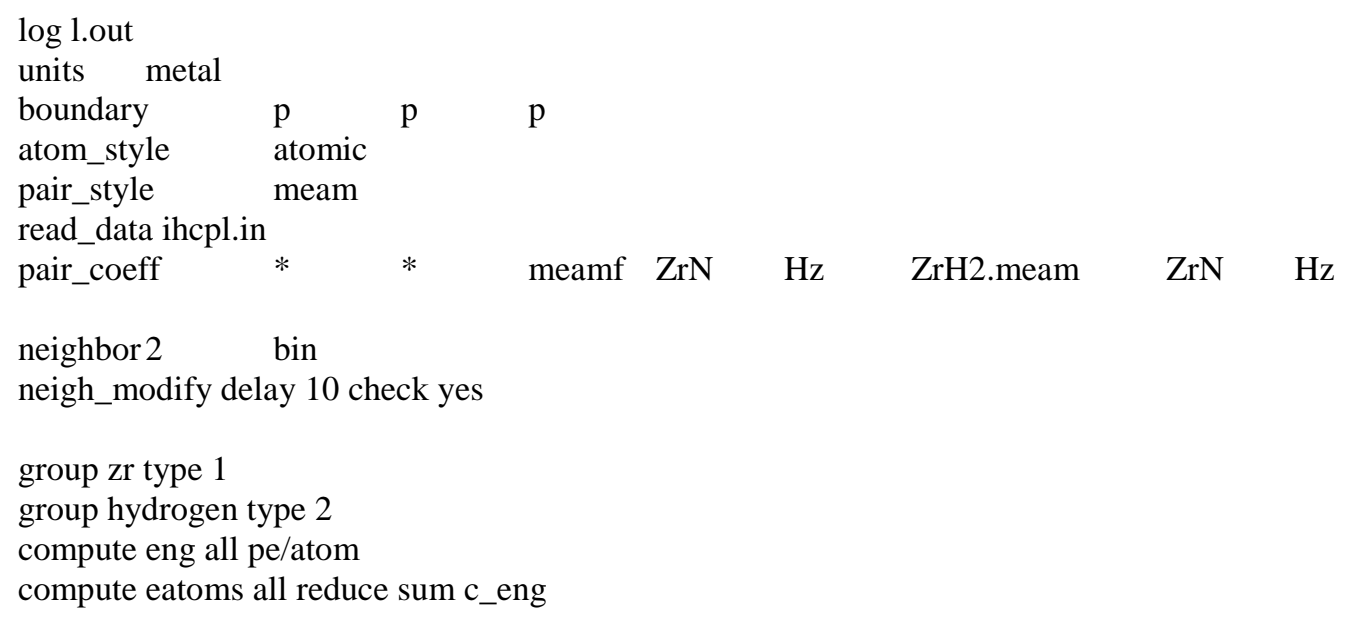




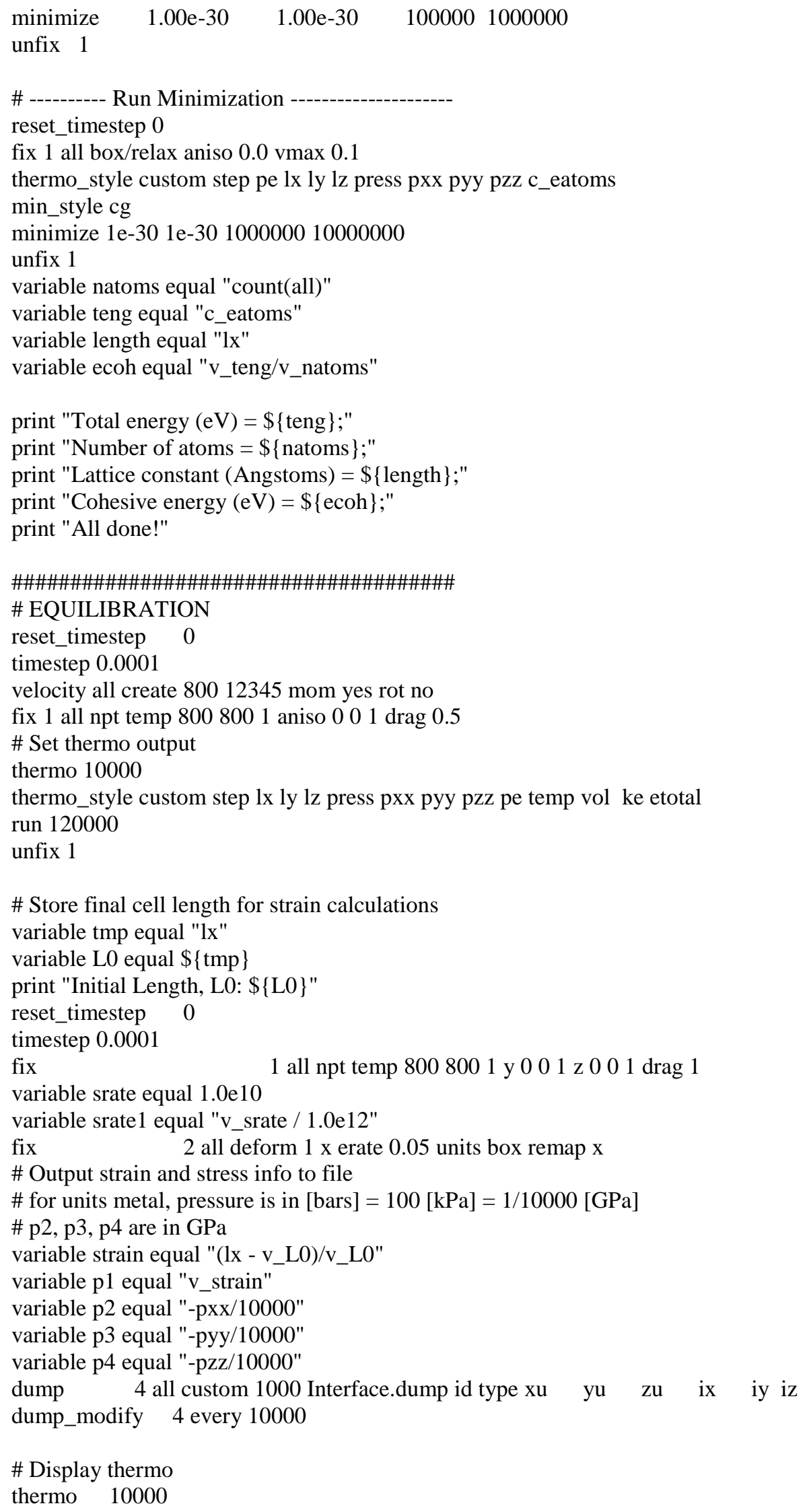


thermo_style custom step v_strain temp v_p2 v_p3 v_p4 ke pe press vol

run 300000

unfix 1

unfix 2

\section{Crack Simulations folder contains calculations for MEAM as discussed in Chapter 9}

\section{Methodology}

Effect of hydrogen on crack propagation is studied in zirconium at various temperatures. A stable crack is created in zirconium. Energy of the entire system is minimized. Temperature equilibration is done on structure. The script here computes stresses around the atoms upon stain. With each step of strain corresponding stresses surrounding each atom is calculated and the configurations are dumped at each change in timestep. The script below is modified from LAMMPS software to provide us the required output

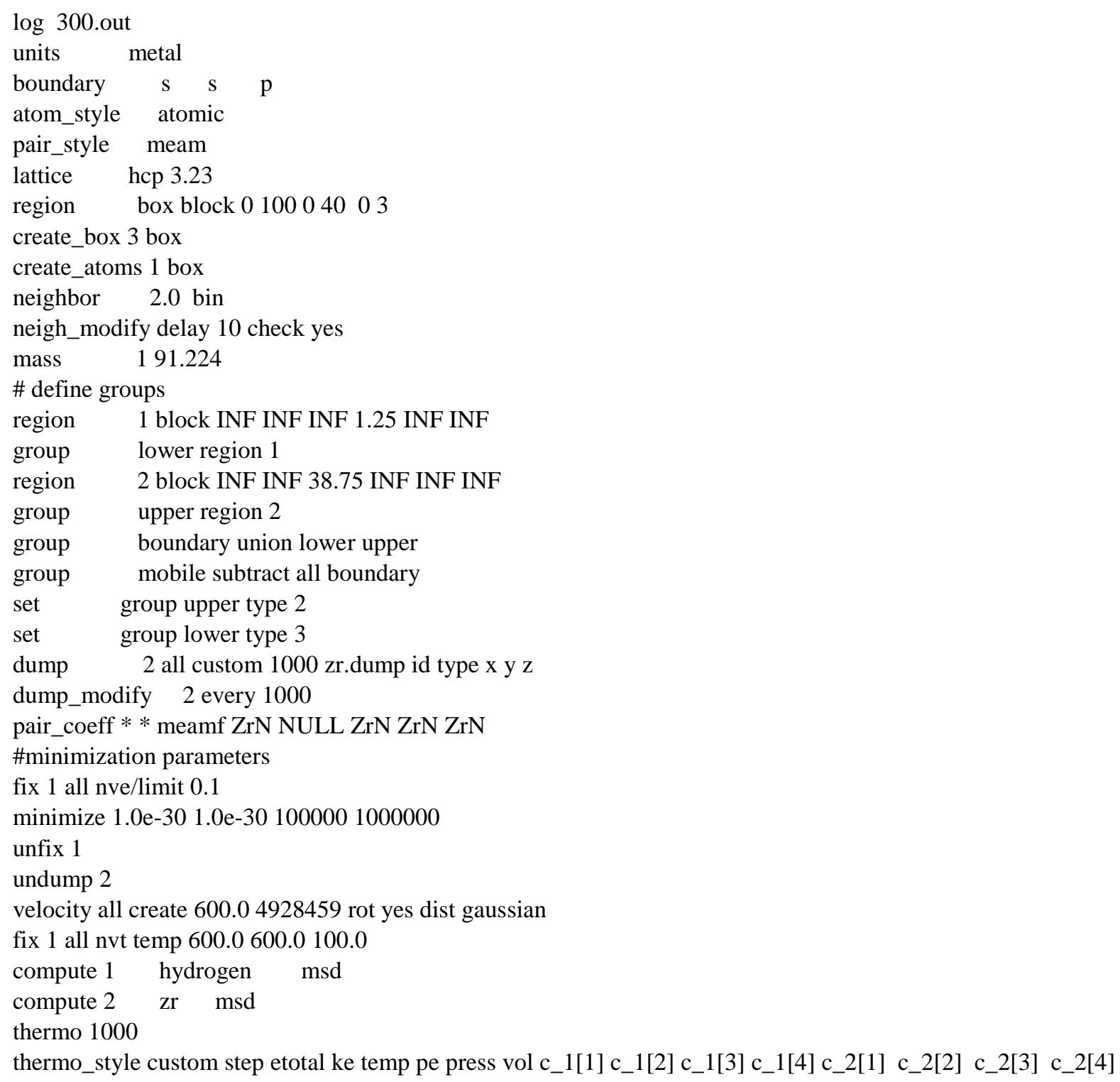




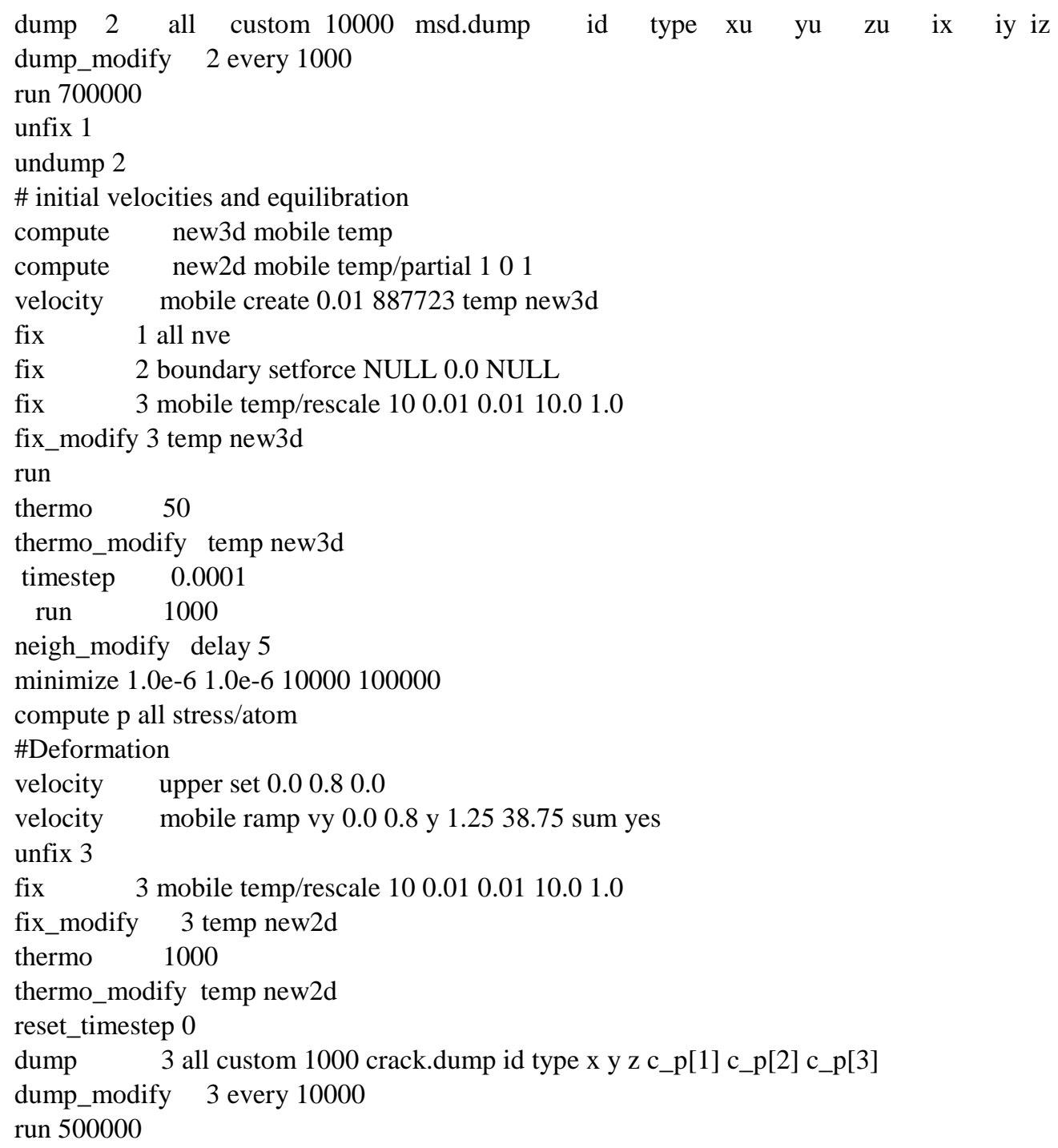

